NOTE TO USERS

This reproduction is the best copy available.

(B)

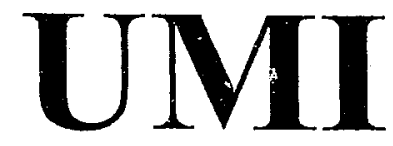

Reproduced with permission of the copyright owner. Further reproduction prohibited without permission. 
Reproduced with permission of the copyright owner. Further reproduction prohibited without permission. 


\title{
HARDWARE SOFTWARE CO-SYNTHESIS OF HETEROGENEOUS HYPERCUBE ARCHITECTURES FOR FAULT TOLERANT EMBEDDED SYSTEMS
}

by

Jacob Levman, BASc, Toronto, September $17^{\text {th }} 2004$

\author{
A thesis \\ presented to Ryerson University \\ in partial fulfillment of the \\ requirement for the degree of \\ Master of Applied Science \\ in the program of \\ Electrical and Computer Engineering \\ Toronto, Ontario, Canada, 2004 \\ (c) Jacob Levman 2004
}

1...2.

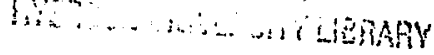

Reproduced with permission of the copyright owner. Further reproduction prohibited without permission. 
UMI Number: EC52963

\section{INFORMATION TO USERS}

The quality of this reproduction is dependent upon the quality of the copy submitted. Broken or indistinct print, colored or poor quality illustrations and photographs, print bleed-through, substandard margins, and improper alignment can adversely affect reproduction.

In the unlikely event that the author did not send a complete manuscript and there are missing pages, these will be noted. Also, if unauthorized copyright material had to be removed, a note will indicate the deletion.

(B)

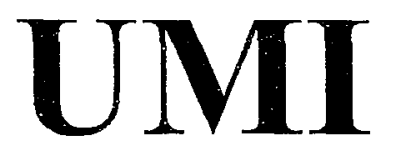

UMI Microform EC52963

Copyright 2008 by ProQuest LLC.

All rights reserved. This microform edition is protected against unauthorized copying under Title 17, United States Code.

ProQuest LLC

789 E. Eisenhower Parkway PO Box 1346

Ann Arbor, MI 48106-1346 
Ryerson University requires the signatures of all persons using or photocopying this thesis. Please sign below, and give address and date. 


\title{
Hardware Software Co-Synthesis of Heterweneous Hypercube Architectures for Fault Tolerant Embedded Systems
}

\author{
Jacob Levman, \\ Masters of Applied Science, 2004. \\ Electrical and Computer Engineering. \\ Ryerson University
}

\begin{abstract}
The hardware-software co-synthesis of an embedded system 's archilecture involves the puttituning of a system specification into hardware and sofiware modules so as to meet varisus min-functional requirements. A designer can specify, many non-functional requirements including cost. performunce. reliability etc. In this thesis, we present an approach to the hardware-soffware co-s!nthesis of emhedded systems targeting hypercube topologies. Hypercube topologies provide a fexible and reliable architecture for un embedded device with multiple processing elements. To the best of our knowledge, this is the first time that hypercube topologies hove been supported in a co-synthesis algorithm. The co-synthesis approach presented here supports the following features: 1) input in the form of an aclclic periodic rask graph with real-time constraints, 2) the pipelining of task graphs. 3) the use of a heterogenesus set of processing elements, 4) Support for fault tolerance through our newly developed group based fisull tolerunce technique. The co-synthesis algorilhm has been applied to wo case studies to demonstrute its efficacy.
\end{abstract}




\section{Acknowledgements}

The author would like to thank his two supervising professors. Dr. Gul Khan and Dr. Javad Alirezaic, for providing their guidance, knowledge and support. The author would also like to thank the members of the review committee for their participation. The author would like to thank the National Science and Enginecring Research Council of Canada (NSERC) for providing funding support for this research project in the form of multiple grants to my supervising professors. The author would like to thank Ontario Graduate Scholarships (OGS) for funding this research through a scholarship. The author would also like to thank Canadian Microelectronics Corporation (CMC) for providing the ARM rapid prototyping platform that was used in the case studies of this thesis. 


\section{Dedication}

I would like to dedicate this thesis to my wife whose drive to accomplish is inspiring.

Without her love and support I would not be where I am today. 


\section{Table of Contents}

1. INTRODUCTION

1.1 Overview 1

1.2 Original Contributions 3

1.3 Thesis Organization 4

2. DESIGN OF EMBEDDED SYSTEMS 6

2.1 Introduction to Hardware-Software Co-Design 6

2.2 Hardware-Software Partitioning 16

$\begin{array}{ll}\text { 2.2.1 Standard Approach } & 17\end{array}$

2.2.2 Partitioning Granularity $\quad 18$

2.2.3 Dynamic Programming 21

2.2.4 Fault Tolerance 22

2.3 Hardware-Software Co-Synthesis 25

2.4 Hypercube Architectures 28

3. CO-SYNTHESIS FOR HYPERCUBE SYSTEMS 33

3.1 Introduction 33

3.2 Fault Tolerance at the Task Graph Level 38

3.2.1 Overview 38

3.2.2 Task Based Fault Tolerance $\quad 39$

3.2.3 Cluster Based Fault Tolerance $\quad 40$

3.2.4 Group Based Fault Tolerance $\quad 44$

3.2.5 Task Graph Based Fault Tolerance Comparison $\quad 49$

3.3 Pipelined Scheduler 51

3.4 Device Expansion 57

3.5 Communication Link Integration 62

4. IMPLEMENTATION AND EXPERIMENTAL RESULTS 68

4.1 Parallel MPEG-2 Decoding 68

vii

Reproduced with permission of the copyright owner. Further reproduction prohibited without permission. 
4.2 Parallel Block Matching 


\section{List of Tables}

Table 3.1: Fault Tolerance Comparison Data

Table 4.1: Processing Element Utilization of Design Space for MPEG Decoding 72

Table 4.2: Processing Element Utilization of Design Space for Block Matching 86

Table 4.3: Gencrated Motion Vectors 92 


\section{List of Figures}

Figure 2.1: Traditional Design 8

Figure 2.2: Hardware-Software Co-Design 10

Figure 2.3: Hardware-Software Co-Synthesis 12

Figure 2.4: $\wedge$ 4D Hypercube 29

Figure 2.5: Examp.e Hypercube Architecture 32

Figure 2.6: Example Hierarchical Architecture 32

Figure 3.1: Hypercube Co-Synthesis Algorithm Flow 37

Figure 3.2: Task Based Fault Tolerance Example $\quad 40$

Figure 3.3: Cluster Based Fault Tolerance - Input \& 1 Iteration 42

Figure 3.4: Cluster Based Fault Tolerance -2 \& 3 Iterations 43

Figure 3.5: Cluster Based Fault Tolerance - Final Clustering $\quad 4.3$

Figure 3.6: Final Added Assertion Tasks After Clustering 44

Figure 3.7: Group Based Fault Tolerance - Input \& 1 Iteration 47

Figure 3.8: Group Based Fault Tolerance - 2 \& 3 Iterations 47

Figure 3.9: Group Based Fault Tolerance - Final Grouping 48

Figure 3.10: Final Added Assertion Tasks After Grouping 48

Figure 3.11: Randomly Generated Task Graph (Graph \#1) 51

Figure 3.12: Hypercube Co-Synthesis Scheduler 54

Figure 3.13: Binary Naming for Hypercube Nodes 63

Figure 3.14: Non-Symmetrical 3-D Hypercube 66

Figure 4.1: Parallel MPEG Decoding: Functional Task Graph 69

Figure 4.2: Design Space Exploration of Parallel MPEG Decoding 71

Figure 4.3: Parallel MPEG Decoding: Architecture for $1^{\text {st }}$ Test Case 73

Figure 4.4: Parallel MPEG Decoding: Architecture for $2^{\text {nd }}$ Test Case 74

Figure 4.5: Parallel MPEG Decoding: Architecture for $5^{\text {th }}$ Test Case 75

Figure 4.6: Block Matching: Functional Task Graph 77

Figure 4.7: Block Matching Task Graph: GBFT Grouping 78

Figure 4.8: Block Matching Task Graph with Added Assertion Tasks 79

Figure-4.9: Block Matching: Final Resultant Task Graph 80 
Figure 4.10: Block Match Circuit Design 81

Figure 4.11: Block Match State Machine 83

Figure 4.12: Design Space Exploration of Parallel Block Matching 85

Figure 4.13: Final Prototype Device Architecture 89

Figure 4.14: Reference Image 90

Figure 4.15: Input Image 91

Figure 4.16: Macroblock Identification 91 


\section{Glossary of Acronyms}

CAD - Computer Aided Design

CiBFT - Group Based Fault Tolerance

CBFT - Cluster Based Fault Tolerance

TBFT - Task Based Fault Tolerance

CPU - Central Processing Unit

ASIC - Application Specific Integrated Circuit

FPGA - Field Programmable Gate Array

MPEG - Motion Picture Experts Group

PE - Processing Element

IP - Intellectual Property

SW - Software

HW - Hardware

VHDI, - Very high speed integrated circuit Hardware Description Language 


\section{CHAPTER 1 \\ INTRODUCTION}

\subsection{Overview}

The average Canadian equates the idea of a computer with a desktop or laptop. In actuality, the definition of a computer is much broader. It is estimated that in the average Canadian's home there are 30 to 40 embedded systems. Television. audio systems, refrigerators, telephones, temperature controls and stoves all use embedded computers. Embedded computers also play an integral role in many of the assisted devices that aid disabled individuals in performing daily activities, e.g. power wheelchairs and communication devices. Additionally, many embedded devices are responsible for protecting human life; embedded computers control modern medical instrumentation, airplanes, air traffic control systems, anti-lock breaking systems (ABS) and even the "fasten your seatbelt" light on the dashboard of your car. By many estimates, embedded devices account for $99 \%$ of worldwide computers while desktops and laptops account for just 1\%. Embedded computers are small-scale application-specific computing devices. Embedded computers already permeate our society and their growth is expected to continue indefinitely.

It is common knowledge that technological developments are producing increasingly efficient and compact computers. This applies to embedded computers as well. The 
more powerful and complicated the components of an embedded derice. the more difficult it is for a computer engineer to produce a product that meets safely. performance, cost and power consumption requirements within a reasonable amount of time. The production of the high performance embedded devices of the future will require tools and formal methods to aid engineers in system design and development.

The research presented in this thesis is centred around the development of computer aided design (CAD) software tools that will, be used to aid in the design and development of future embedded devices. Essentially, an engineer will tell the CAD tool what the desired device should be capable of doing; the tool will analyze the given information and recommend a reliable and efficient design. This can aid a product engineer in developing systems that are far more reliable, cost, time and power efficient. Reliability is key for the development of safety-oriented devices that are responsible for protecting human life. Minimal production cost is important for ensuring the final products are accessible to all people regardless of socio-economic status. Similarly, decreased labour costs as a result of more expedient design, results in a cheaper product. Finally, minimizing a device's power consumption is essential to reducing energy costs, both financial and environmental. These CAD tools will aid in the development of new devices that will continue to play a central role in our lives.

Within electrical and computer engineering, embedded systems research is in its infancy. This makes it an exciting field to work in as it provides unique and novel 
opportunities. It is also attractive due to its broad range of applications (acrospace, automotive, communications etc.). Developing these software tools can be technically challenging and thus intellectually rewarding.

\subsection{Original Contributions}

This thesis presents a new hardware-software co-synthesis tool to aid in the design and development of high performance embedded devices. The proposed approach focuses primarily on computationally intensive computing systems requiring high levels of fault tolerance. A full hardware-software co-synthesis approach is presented with comparisons to a fully exhaustive technique. Implementation results are also provided in order to further demonstrate the algorithm's efficacy.

The major contributions of this thesis are as follows:

- Development of a hardware-software co-synthesis algorithm capable of generating hypercube architecture based embedded devices

- Development of group based fault tolerance (GBFT), a technique designed to effic $\ldots . . t$ tly add support for fault tolerance in embedded systems at the task graph level

- Comparative analysis between the newly developed GBFT algorithm and other existing methods

- Comparative analysis between the co-synthesis algorithm presented here and 
the optimal case

- Implementation of a security/navigation device in simulation in order to demonstrate the efficacy of the co-synthesis algorithm

- Implementation of a parallel block matching device in order to demonstrate the efficacy of the co-synthesis algorithm

- $\quad$ Prototype construction of the block matching case study

\subsection{Thesis Organization}

This thesis consists of five chapters. The second chapter encompasses a survey of hardware-software co-design, and a thorough survey of hardware-software partitioning, and co-synthesis. Chapter 2 also includes background on hypercube architectures. These two chapters are intended to provide the basic understanding of the design issues of hardware-software embedded systems and to survey the existing research in this field.

The third chapter is the main component of this thesis. It consists of a full description of all of the components of the co-synthesis algorithm. These include group based fault tolerance, a pipelined scheduling technique, a method for adding processing elements to the current system design, placing existing processing elements within a hypercube topology and synthesizing all required communication links. 
The fourth chapter describes the two case studies implemented in order to demonstrate the algorithm's efficacy. The first case study performs the decoding of parallel MPEG-2 video streams and compares the algorithm's results with that of an exhaustive technique. The second case study performs parallel block matching and a final prototype device is constructed. The fourth chapter also includes a discussion of the experimental results obtained from both of the case studies. The fifth chapter concludes this thesis. 


\section{CHAPTER 2}

\section{DESIGN OF EMBEDDED SYSTEMS}

\subsection{Introduction to Hardware-Software Co-Design}

This section surveys hardware software co-design. Hardware-software co-design is an active area of research that involves the development of tools and methodologies to aid in the design of embedded computer systems. Embedded computers are processing devices used in areas as diverse as wireless communications, medical instrumentation, transportation and food preparation. Although these fields are widely different, the embedded device components of the products share common design techniques. This is an outline of hardware-software co-design: a method for jesigning and developing an embedded computer device. The motivation behind codesign is that both hardware and software components should be addressed simultaneously in order to ensure that the final device meets cost, performance, reliability and power consumption goals.

Separate software and hardware design methods have been the subject of a great deal of research over the years [21, 41]. However, the design of both hardware and software as a joint venture remains an area of rapidly growing research. Most of the embedded systems research has been stimulated by the development of fairly inexpensive high performance microprocessors [8]. When embedded processors were 
exclusively small and responsible for the execution of minimal amounts of software, simple techniques were more than sufficient to create devices that satisfied performance and functional goals within a reasonable time to market. With the number of transistors on a chip increasing exponentially, embedded devices have the potential to utilize far more sophisticated circuits and architectures [48]. The embedded engineer requires $\mathrm{CAD}$ tools to aid in the design and development of embedded computers and to predict implementation costs.

The rest of the subsection introduces the motivation behind hardware-șoftware codesign of embedded systems. Additionally, it intends to introduce the various components of the hardware-software co-design process.

\section{Co-Design Overview}

The embedded system design process will vary considerably with respect to the type of product under development. However, commonalities can be identified and the ability to abstract hardware and software components to the same level is greatly exploited in hardware-software co-design. The traditional approach to the design of an embedded computer system is to enforce hardware-software partitioning at an early stage. This results in well-defined design tracks for bonth the hardware and software components. The major weakness in traditional embedded systems design 
lies in the early partitioning process. A graphical overview of the traditional design approach is provided in Figure 2.1 below.

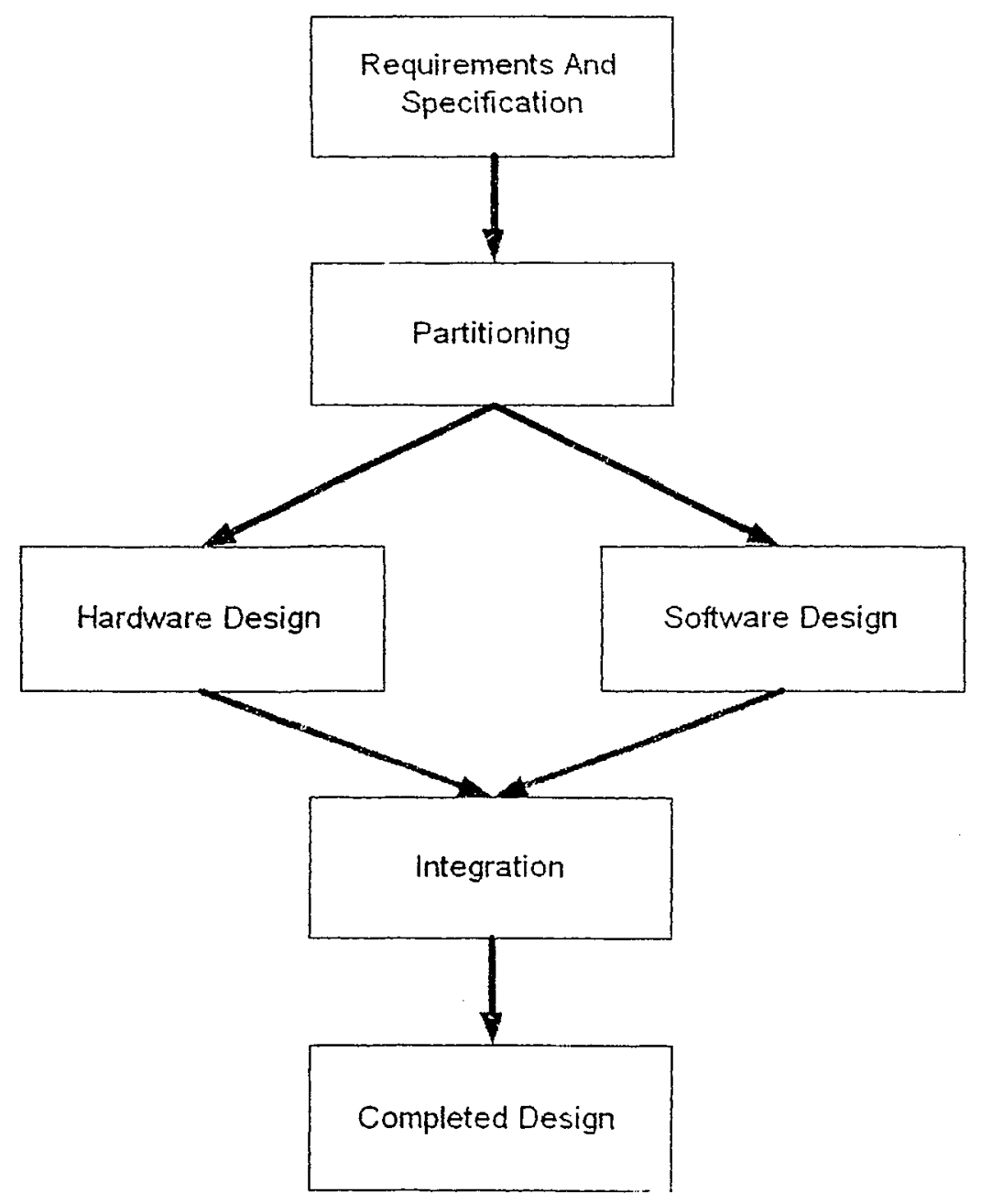

Figure 2.1: Traditional Design

One of the major flaws of such an approach is the inability of the design and development flow to correct mistakes made in the partitioning phase. If during integration, an embedded systems engineer discovers that the product nill not meet 
various non-functional requirements (performance, power consumption, etc.), the cost imposed on re-evaluation of the design will be extremely high.

The hardware-software co-design process begins with the creation of device requirements, which leads to a formal specification. Both functional and nonfunctional requirements such as performance, cost and power consumption are specified. This can then be converted into a standardized system description or specification. Embedded system specification requires detailed models to aid in the abstract description of component functionality. Abstract modeling that does not differentiate between hardware and software is known as co-specification. Further research into the high level modeling of embedded devices would be greatly beneficial.

It is common for this standardized description to be converted into a task graph format. Hardware-software partitioning is performed on this task graph. Partitioning is concerned with assigning an execution location (software or hardware) to each task. After partitioning, co-synthesis is performed and typically, the co-synthesis and partitioning phases are closely knit. Co-synthesis is broken down into the assignment, allocation and scheduling phases. Finally, the generated software, hardware and interface modules are integrated. Feedback in the design process can occur at system integration by returning to the partitioning phase, thus allowing the designer to refine the given solution. At integration, the overall system can be evaluated for functional and non-functional requirements by using hardware software 
co-simulation. Co-simulation allows for both hardware and software components to be tested congruently. A visual overview of the co-design process is provided in Figure 2.2

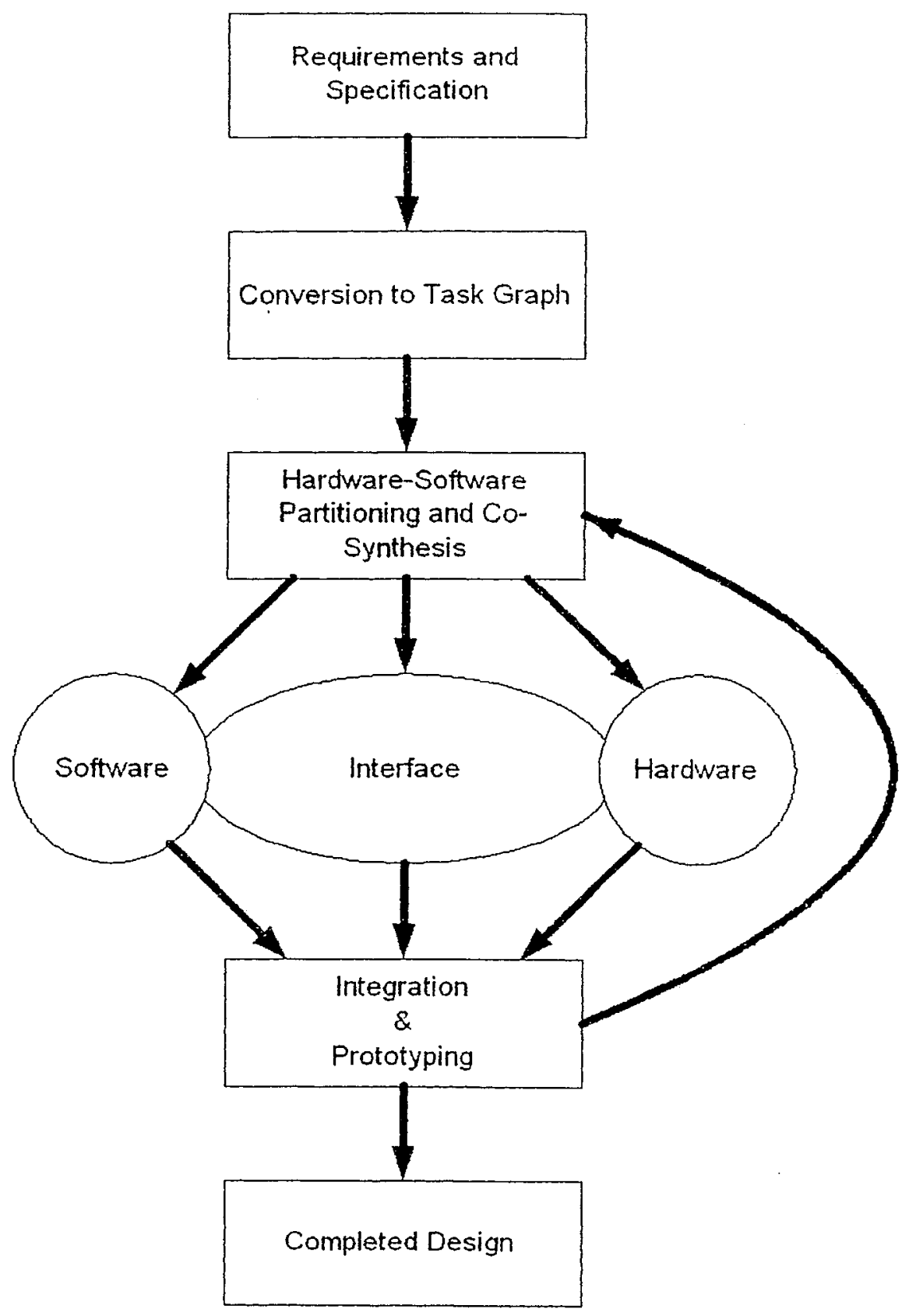

Figure 2.2: Hardware-Software Co-Design 


\section{HW/SW Partitioning}

The partitioning process is concerned with deciding what system functionality will be implemented as hardware and what will be implemented as software. Typically, an embedded device will need to meet a number of non-functional requirements. These would often include performance, price, reliability and power consumption. With more components implemented in hardware, system price and power consumption will increase. However, with a large number of components implemented in software, system performance and reliability can degrade. It is important to balance the selection of hardware and software components to ensure that all system requirements are met. Significant research has been conducted with respect to partitioning algorithms in order to automate the process of obtaining an efficient hardware-software layout for an embedded device.

\section{HW/SW Co-Synthesis}

Hardware-software co-synthesis of an embedded device is the process by which the hardware-software architecture of the system is automatically derived to satisfy multiple goals. These goals can include factors such as cost, performance and power consumption. Hardware-software co-synthesis is inseparable from the process of 
partitioning. The hardware-software co-synthesis design flow consists of three main components: allocation. assignment and scheduling. Allocation is concerned with selecting the number and type of communication links and processing elements in the system. The assignment component is concerned with the mapping of tasks to processing elements. The scheduling component is concerned with the timing of task execution and communications. Typically, the partitioner will iteratively adapt its hardware mappings based largely on the results of the scheduler. The scheduler is typically the final phase of co-synthesis. A visual overview of a common approach to co-synthesis is provided in Figure 2.3.

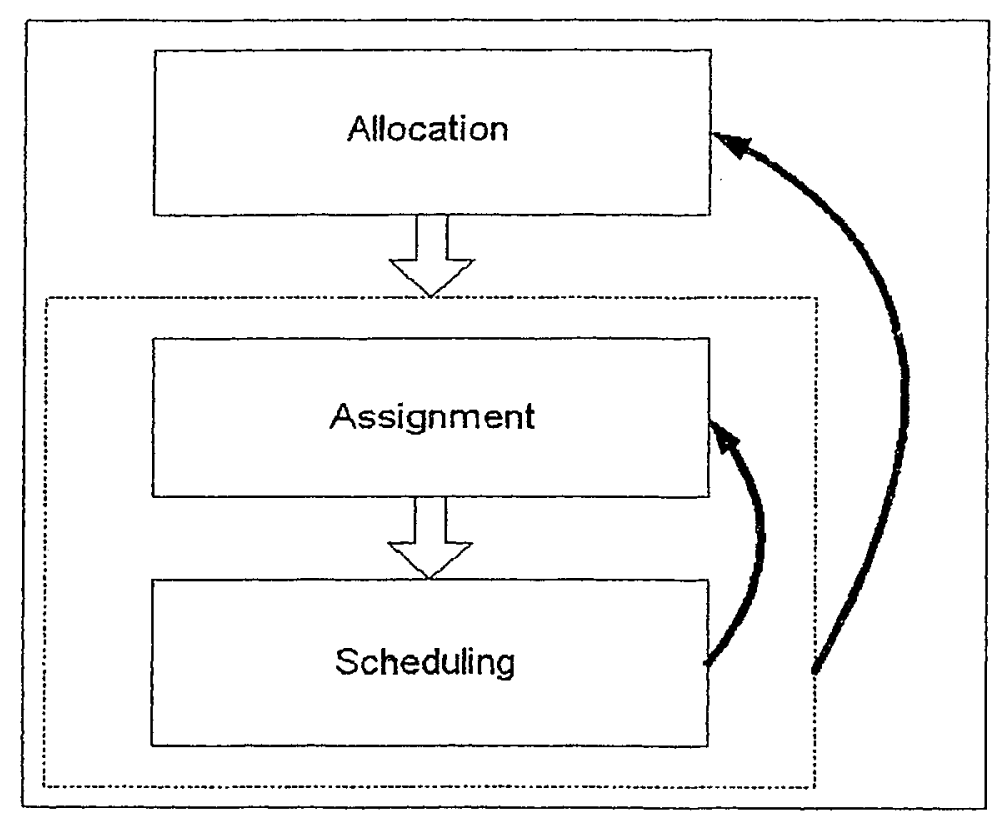

Figure 2.3: HW/SW Co-Synthesis 


\section{HW/SW Co-Simulation}

High performance embedded system components can be extremely complex. It is difficult to develop comprehensive analytic systems to model the performance of an embedded device that consists of complicated components. Concurrently simulating components with differing behavioural models is referred to as co-simulation. Typically, a co-simulation environment will model multiple components, both software and hardware. This can be a difficult task as software simulation consists of modeling a processor executing a series of instructions. However, hardware simulation can consist of modeling something completely different, such as an analog or digital circuit. In an embedded device it is common for the execution of application specific integrated circuits to depend on commands issued by one of the system's processors. One should note that co-simulation requires the hardware simulator to react to input from the software simulator and vice versa.

A number of co-simulation tools have been developed. One of the most well-known co-simulation tools is Seamless from Mentor Graphics. Seamless allows the user to tie in various hardware and software simulators. Seamless coordinates the communications between simulators to ensure that the overall behaviour reflects that of an embedded device. Although Seamless performs as an effective co-simulation tool, it can also be applied for hardware-software co-verification [44]. 


\section{HW/SW Co-Verification}

The complexity of embedded systems prevents the designer from relying on traditional validation techniques such as simulation and testing. These techniques are insufficient to properly verify the correctness of such a system. To address this problem, new formal verification methods are needed to overcome the limitations of traditional validation techniques.

Multiple methods have been developed for performing hardware-software coverification. A common approach involves the use of a Petri-net based representation of embedded systems, as in the system named PRES [9, 10]. The PRES model proves the correctness of an embedded system by determining the truth of computation tree logic and timed computation tree logic. These research projects make use of model checking to prove the correctness of embedded systems and have used an automatic teller machine server to demonstrate the feasibility of their approach. Another approach developed by Hsiung involves the use of linear hybrid automata and employs a simplification strategy to address the state-space explosion that occurs in the formal verification of complex systems [26].

Presently, our knowledge of the joint hardware-software design process is far more limited than that of either of the two separately. While embedded systems development can be performed as separate processes of hardware and software 
design, this route can be far more challenging when attempting to meet various price and performance rer $\quad$ sunts. Additionally, this design path has a tendency to result in more error prone ucts due to difficulties in embedded system verification.

Research into system modeling is a key element to our understanding of hardwaresoftware co-design. While many abstract models for embedded system components exist, there is a lack of accurate models to address the detailed characteristics of these components. Embedded systems are always being developed with various performance and cost metrics in mind. In order to properly meet those requirements, it is essential to develop a thorough understanding of modeling that includes both the intricacies of a component as well as its high level properties.

In the current embedded systems market, designers and developers can mostly produce devices that meet requirements within a reasonable amount of time. However, soon the utilization of sophisticated hardware-software co-design techniques will be required in order to meet future device demands. As advanced processors and ASICs become less expensive, the need for tools to aid the design and development process will increase dramatically in order to ensure the development of high quality devices with a minimum time to market. 


\subsection{Hardware-Software Partitioning}

Hardware-software partitioning is the process by which the various components of an embedded computer's functionality are placed in either hardware or software. The motivation behind the partitioning process is to produce a reliable embedded device that meets performance, cost and power consumption requirements.

The partitioning process is a subset of hardware-software co-design. Computer components suitable for use in embedded devices have increased in ability and complexity dramatically in recent years. The job of an embedded systems engineer involves selecting appropriate components and integrating them to produce an embedded device. The rapid increase in both complexity and performance of these components has resulted in an increase in the difficulty of component selection and integration. These difficulties have fuelled demand for tools and design methodologies to aid in the creation of embedded devices that are comprised of both hardware and software components.

The typical embedded system design approach is significantly restrictive. The main flaw revolves around the lack of design flow after system integration and prototyping. If the system is integrated and an expensive prototype is produced, further design changes can be extremely costly. These further design changes may have to occur if 
prototyping reveals that the system will not meet non-functional requirements (such as performance).

This subsection`s goal is to introduce the reader to the motivation behind hardwaresoftware partitioning for embedded devices. Additionally, this subsection intends to introduce the reader to the existing research in hardware-software partitioning.

\subsubsection{Standard Approach}

The partitioning process is concerned with deciding which system functionality will be implemented as hardware and which will be implemented as software. It is important to balance the selection of hardware and software components to ensure that all system requirements are met. Significant research has been conducted with respect to partitioning algorithms in order to automate the process of obtaining an efficient hardware-software layout for an embedded device.

The standard approach to hardware-software partitioning involves the use of a heuristic to prioritize a task set. This prioritization aids in the determination of task mapping (to hardware or software). Often much of the job of the partitioning algorithm researcher is simply to develop an effective heuristic that will result in an optimal partitioning algorithm. 
The earliest partitioning algorithms proposed, began by implementing all components in software and proceeded to move components to hardware implementations until various system requirements were optimized [18]. Other early approaches proposed to implement all components in hardware and to proceed to move components to software until system requirements were met [22]. A more recent approach involves making an educated guess with reference to whether a given task should first be mapped to software or to hardware [6]. Afterwards, the algorithm would iteratively attempt re-mapping tasks from their original locations until requirements are satisfied. These techniques and versions thereof are still in use. In all cases the approach is similar, the algorithm attempts a default or initial configuration, analyzes its effectiveness and iteratively alters the current layout if non-functional requirements are not met. Other techniques to aid in the heuristics for partitioning decisions include linear integer programming [38], simulated annealing [40] and petri-nets [19].

\subsubsection{Partitioning Granularity}

Granularity defines the size of system components that can be implemented in either hardware or software. A partitioning algorithm that operates at a high level of granularity (also referred to as coarse-grained) uses only large blocks of system functionality to be implemented on any given processing element (PE). It can be beneficial for a large segment of functionality to be implemented on the same PE. This is particularly evident in larger scale distributed embedded devices, where 
dividing a high grained system into smaller components and then mapping them across the system can yield an unnecessarily high communication overhead. This occurs when tightly coupled components are "spread out" throughout the system. Using the granularity that is specified by the programmer of an application is often referred to as a high level of granularity. Gupta and DeMicheli developed an approach to the coarse grained hardware-software partitioning problem [23]. They present a partitioning procedure to identify potential hardware and software components of a system. Their technique also utilizes the Olympus Synthesis System for digital design [39] for the synthesis of dedicated hardware within their system. Yen and Wolf have also developed an approach to the coarse grained partitioning problem [52]. They present an automatic iterative improvement technique for simultaneously performing concurrency optimization and hardware-software tradeoffs. By considering both concurrency and hardware-software tradeoffs, their approach is able to identify cost/performance points that may not have been identified otherwise.

A partitioning algorithm that operates at a low level of granularity (also referred to as fine-grained) will often divide an embedded system's functionality into extremely small components. Fine-grained partitioning algorithms have the disadvantage that separating system functionality on such a small scale can dramatically increase communication overheads, which has the effect of decreasing system performance. Some systems use this approach when they are dealing with partially re-configurable processors (processors whose IP cores can be modified during the design process). 
Fine-grained systems can provide a better solution than coarse-grained algorithms because they are more llexible in terms of mapping the correct computationally intensive components to the appropriate processing elements. Fine-grained systems reduce the potential negative effect of having to deal with a poorly defined system functional specification. A fine-grained system can refine the design on such a small scale that some will take one single computationally intensive CPU instruction and treat it as a separate task [1]. It is common for tasks (individual components that can be mapped to hardware) to be called base blocks when dealing with fine grained partitioning. Ernst et al. have developed a heuristic approach to the fine grained hardware-software partitioning problem [18]. They have developed an iterative partitioning process which is based on hardware extraction and is controlled by a cost function. This technique is in use in the COSYMA system [40]. Knudsen and Madsen have also presented an approach to the fine grained partitioning problem [30]. This approach uses dynamic programming to solve both the problems of system execution time and hardware area constraints. This technique is in use in the LYCOS system [38].

Finally, there is one research project known that involves merging these two ideas of varying granularity [24]. This concept is known as flexible granularity. Depending on the characteristics of the specific application and the system's non-functional requirements, the selected granularity can span from a low level of base blocks all the way to the user-defined functions. This approach is intended to overcome the shortcomings of both of the previous approaches. This work also includes estimation 
methodologies adapted to different levels of granularity, which help to determine the final system granularity.

\subsubsection{Dynamic Programming}

Dynamic programming is a technique ideal for problems where calculating all possible outcomes is not computationally feasible. This makes dynamic programming well suited to the partitioning problem, which can be extremely computationally intensive. Typically, a dynamic solution is recursive and iterative in nature.

Dynamic programming problems can always be divided into stages where a decision will be required at each stage. Typically there are a number of states associated with each stage. Decisions made at one stage will alter the current state into a new state in the next stage. The decision made at a given state does not depend on the decisions made in the previous state. It can be seen that dynamic programming extends itself easily to hardware-software partitioning which can be approached as a recursive, iterative, state based problem. Often a dynamic programming solution will effectively process a task graph and improve algorithm execution speed by avoiding testing infeasible combinations. 
Shrivastava et al. [45] have used dynamic programming to develop an algorithm that can effectively solve the partitioning problem with extremely fast execution times. Chang and Pedram [5] have also used dynamic programming to determine the solution to the coarse-grained partitioning problem of a generic task graph. Knudsen and Madsen [31] have used dynamic programming to determine the solution to the fine-grained partitioning problem.

\subsubsection{Fault Tolerance}

Fault tolerance is a large area of computing, whereby computational devices are developed which must meet various mission critical and safety critical requirements. These types of systems are common in aerospace and biomedical applications. Fault tolerant computing has been a large area of study [46], however, incorporating some of these ideas into hardware-software partitioning is an extremely young discipline.

It is common in the development of fault tolerant devices to incorporate a system's fault tolerant components late in the design process. This often creates a significant overhead in terms of implementation cost. Incorporating fault tolerance at an earlier stage of design is likely to be very beneficial in reducing this overhead. This line of reasoning has sparked research in incorporating fault tolerance inito earlier phases in the design process, such as hardware-software partitioning. 
Fault tolerant hardware-software partitioning generally involves adapting the partitioning process to automatically accommodate systems requiring fault tolerance. The most predominant work in this field is that of Dave and Iha [13]. These researchers have developed a system that will input a functional task graph specification and perform task clustering for fault tolerance. Their system will choose an error recovery topology that is optimized to use a small number of extra processing elements. Additionally, Bolchini et al. [4] have developed a partitioning algorithm that incorporates fault detection capabilities. This may not be as robust as full fault tolerance, however fault detection is relevant as it is a subset of fault tolerance.

Presently, our knowledge of the joint hardware-software design process is far more limited than that of either of the two separately. While embedded system development can be performed as a separate process of hardware and software design, this route can be far more challenging when attempting to meet various price and performance requirements. Additionally, this design path has a tendency to result in more error prone products due to a lack of formalism in design methods.

Hardware-software co-design is a burgeoning field of research and one of its most active subsets is hardware-software partitioning. Partitioning is the process of deciding what functional system components will be implemented as hardware and what will be implemented as software. Effective hardware-software partitioning is 
essential for creating embedded devices that meet non-functional requirements such as performance, power consumption and cost.

Since hardware-software partitioning has begun to flourish as a field of research, greatly varying approaches to the problem have been taken. Researchers have attempted to tackle many issues and incorporate many varying ideas. The concept of varying degrees of granularity allows an algorithm to separate a system's functionality into components of various size. Dynamic programming is an iterative, recursive technique that has been effectively incorporated into approaches to partitioning. Fault tolerance is an extremely important issue, especially in embedded systems, and partitioning approaches have been developed to incorporate these abilities.

In the current embedded systems market, designers and developers can mostly produce devices that meet requirements within a reasonable amount of time. However, soon the utilization of sophisticated hardware-software co-design techniques will be required in order to meet future device demands. As advanced processors and ASICs become less expensive, the need for tools to aid the design and development process will increase dramatically in order to ensure the development of high quality devices with a minimum time to market. Hardware-software partitioning will be an integral component of these tools. 


\subsection{Hardware-Software Co-Synthesis}

An embedded system's architecture is typically determined by the intuition of a design engineer. This process adds time to the development cycle and sometimes results in architectures that do not meet non-functional requirements. It can also result in an over-designed architecture with excessive hardware that results in overly expensive devices. Consequently, incorporating hardware-software co-synthesis in design automation tools is essential for producing optimal devices with an accelerated time-to-market. The hardware-software co-synthesis problem is concerned with determining optimal hardware and software architectures. This involves the selection of processors (CPUs), application specific integrated circuits (ASICs) and communication links in order to produce a device that meets non-functional requirements. The co-synthesis problem typically involves the selection of the quantity and type of processing elements and communication links (allocation), task assignment from a task graph to processing elements and confirmation of whether the system meets requirements (usually through scheduling). A task graph is a collection of tasks and communication dependencies that describe device functionality. The allocation and scheduling phases are known to be NP-complete $[20,33]$. thus determining an optimal solution in the co-synthesis phase can be extremely computationally intensive.

This work is motivated by the need to automate the embedded system design process while simultaneously producing high quality devices. The research presented in this 
thesis is further aimed at producing high performance fault tolerant embedded systems. Embedded applications that would particularly benefit from high levels of fault tolerance include aerospace, medical instruments and high performance telecommunication systems. In fact, it is believed that within the next two or three decades probes will be sent to orbit nearby stars [34]. Such embedded devices would be responsible for considerable system control and measurement instrumentation in addition to unparalleled fault tolerance requirements.

The primary focus of most of the co-synthesis research problem has concentrated on the simplistic single processor and ASIC architecture $[3,6,18,28]$. Some approaches assume more complicated architectures, such as two CPUs and hardware-accelerated circuitry [37]. Various approaches have been attempted that involve moving tasks from hardware to software or vice versa in order to meet the system requirements. In the co-synthesis of distributed systems, target architectures can incorporate multiple processors, ASICs or FPGAs (field programmable gate arrays). Two nain techniques have been utilized to handle the co-synthesis of distributed systems: the optimal and heuristic approaches.

The optimal approach can be divided into three sections: exhaustive, mixed integer linear programming, and constraint solving. The exhaustive approach is characterized by attempting all possible mappings to provide an optimal solution. It can be very computationally intensive and is only suitable for smaller systems. D'Ambrosio and Hu have presented an exhaustive technique for hardware-software 
partitioning [11]. However, their approach is limited to single processor architectures and ignores the communication overhead. A hardware-software partitioning technique using mixed integer linear programming is presented by Prakash and Parker [42]. The execution time of this technique is prohibitive for large lask graphs. Moreover, this approach is limited to bus based architectures or pre-determined pointto-point interconnection topologies. A constraint solving approach has been presented by Kuchcinski in the JaCoP system that concentrates on scheduling and resource assignment [32].

The heuristic-based co-synthesis approach can be divided into two methods: constructive and iterative. The iterative scheme is characterized by having an initial solution, which is iteratively improved. Kirovski and Potkonjak presented an iterative algorithm that includes power as a cost function but their approach ignored the inter-task communication time [30]. Other iterative techniques have been developed [25] but they are limited in that they allow for only one type of communication link. Li and Wolf have presented an iterative co-synthesis algorithm capable of synthesizing memory hierarchies for bus architecture topologies [36]. MOGAC is an iterative approach to the co-synthesis problem that uses genetic algorithms [16]. Experimental results show that for large systems MOGAC suffers due to large execution times. Wolf has also presented an iterative approach to the cosynthesis problem producing generic device architectures [47]. Generic device architectures can be tuned to a particular application, but it can also result in disorganized and difficult to understand designs as the interconnection topology may 
not conform to an established architecture. The constructive heuristic method is characterized by building the solution step by step. where the final output is not available until the algorithm terminates. Constructive co-synthesis algorithms are presented in the COSYN [15] and COFTA [13] systems. Bakshi and Gajski have also presented a constructive partitioning approach that supports pipelining at varying degrees of granularity [2]. Although their approach allows the addition of multiple software processors, it does not account for the hardware cost of adding each CPU. All of the above co-synthesis algorithms support distributed embedded systems but none have utilized hypercube topologies as a target architecture.

\subsection{Hypercube Architectures}

Hypercube topologies are useful for high performance embedded systems and have a number of advantages over other architectures [12, 27, 35, 43]. Hypercube nodes represent processing elements (PE) and a link between nodes represents a communication interface (serial, parallel, Ethernet link, etc.). Hypercube topologies are very flexible, versatile and generic. Figure 2.4 shows a hypercube network of degree four, constructed from two 3D hypercubes. The high level of interconnectivity in a hypercube architecture results in a system that is suited to fault tolerance. Additionally, hypercube systems support high performance computation while limiting the communication overheads and/or bottlenecks associated with largescale systems consisting of many PEs. These features make hypercube architectures 
an excellent choice for multiple PE embedded systems that need to couple faulttolerance with complex computation. Additional advantages of hypercube architectures include topologies with small diameters and high levels of symmetry. Finally, routing in hypercube networks is well researched and efficient routing algorithms are available [29].

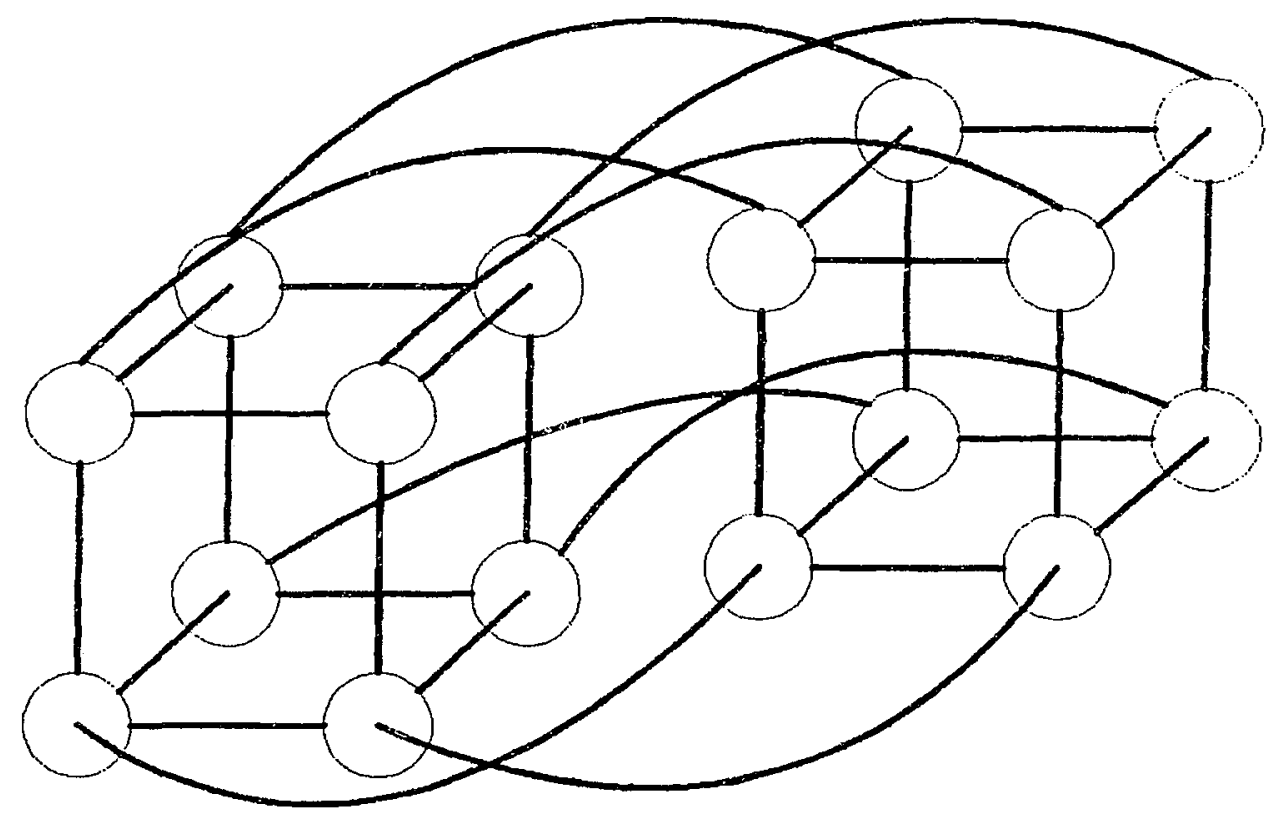

Figure 2.4: A 4D Hypercube

The main disadvantage of hypercube topologies is their poor upward scalability. It can be a difficult and complex process to add nodes to a hypersube network. This criticism applies more directly to hypercube computer systems, which are likely to be reconfigured and expanded regularly. It is uncommon for a distributed embedded device that has already been manufactured to require any additional processing 
elements. Such a circumstance could be used in reconfigurable space systems. Another disadvantage of hypercube systems is the large number of communication links, but additional links support fault tolerance. These communication links and their interfaces to the processing elements result in a significant cost overhead. However, it should be noted that this research targets high performance fault-tolerant embedded devices. As a result, the associated cost to produce systems that meet the flexibility, reliability and performance requirements of demanding applications is knowingly accepted.

Many co-synthesis methods for distributed embedded systems target varying architectures. Hypercube topologies can be considered a superset of a number of other hierarchical architectures. Topologies such as mesh, torus, binary and quad trees can be partially represented by suitable sized hypercube topologies. Binary trees have even been embedded in incomplete hypercube systems [51]. A hierarchical architecture (e.g. tree topology) is one of the most prevalent system targets in the high performance distributed co-synthesis research projects $[13,14]$.

Much of the work on automatic architecture generation in co-synthesis algorithms for distributed embedded systems has concentrated on hierarchical topologies [14]. Although hierarchical systems can be adapted to enhance their fault tolerant capabilities [13], a comparison of the two architectures is provided to illustrate the capabilities of hypercube topologies. Consider a 3D hypercube with eight processing elements presented in Figure 2.5. If the communication link between $\mathrm{PE}^{0}$ and $P E^{1}$ 
fails. the device could still operate correctly by routing messages along alternate channels (e.g. $\left.\mathrm{PE}^{()} \rightarrow \mathrm{PE}^{4} \rightarrow \mathrm{PE}^{5} \rightarrow \mathrm{PE}^{\prime}\right)$. System performance would decrease as communication between nodes connected by a faulty link (e.g. $\left.P E^{0}-P E^{\prime}\right)$ would take longer (three transfers instead of one). However, most importantly. the system will continue to function. Now consider a possible hierarchical architecture implementation of the same embedded device shown in Figure 2.6. If the equivalent communication link (connecting $\mathrm{PE}^{0}$ and $\mathrm{PE}^{\prime}$ ) fails, $\mathrm{PE}^{\prime}, \mathrm{PE}^{3}$, and $\mathrm{PE}^{5}$ of the embedded device would cease to function as they cannot communicate with any of the other PEs. This would result in a catastrophic system failure. The benefits over alternative architectures were a major motivation for developing the first hardwaresoftware co-synthesis algorithm targeting hypercube topologies. 


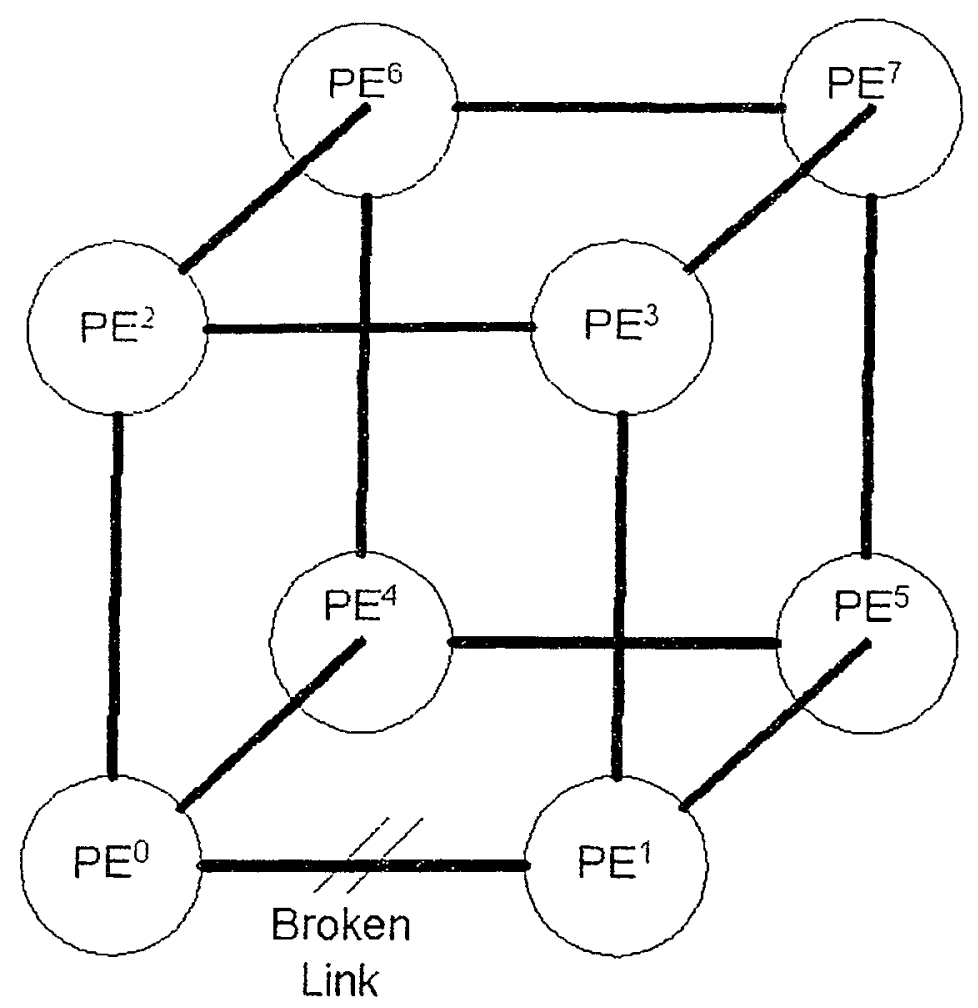

Figure 2.5: Example Hypercube Architecture

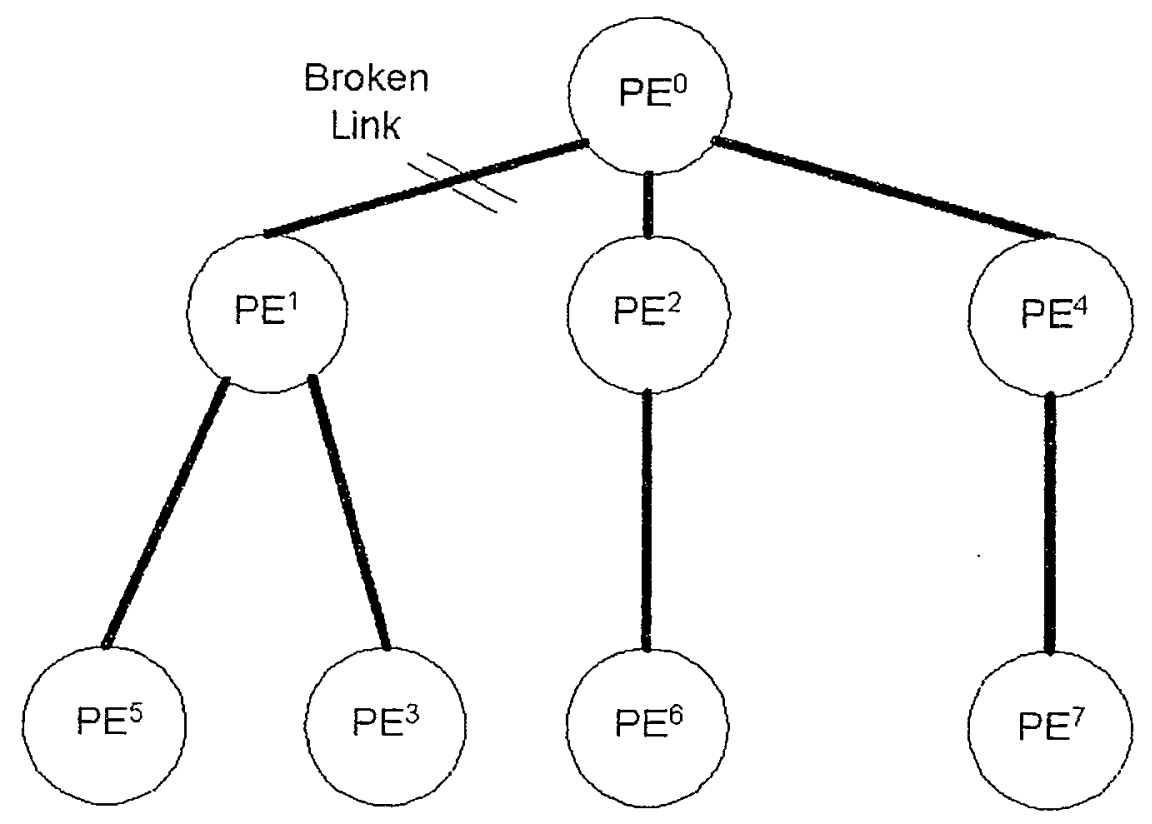

Figure 2.6: Example Hierarchical Architecture 


\section{CHAPTER 3}

\section{CO-SYNTHESIS FOR HYPERCUBE SYSTEMS}

\subsection{Introduction}

A constructive co-synthesis approach that targets a hypercube topology as the final system architecture is presented. The algorithm uses a library of processing elements (PEs) including processor cores (CPUs) and application specific integrated circuits (ASICs), which provides relevant data such as hardware area requirements and performance information. The library can consist of many CPU types and ASICs. The library also provides information related to various types of communication links available with their interconnectivity costs. The algorithm takes in a task graph representing the functionality of the device. The communication links are generic and the co-synthesis algorithm supports all types of communication links including serial, parallel, etc. The algorithm assumes that each PE in the hypercube system consists of either a CPU or an ASIC. In addition to this, each PE consists of some local memory for computational purposes and interface circuitry for communication links.

The constructive co-synthesis approach presented in this thesis is provided in Figure 3.1 and consists of six main steps:

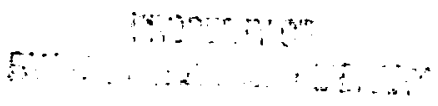


1) Specification: Defining the required device functionality and performance and arca requirements.

2) Profiling: Evaluating each functional unit in the specification for performance and area utilization data.

3) Group Based Fault Tolerance (GBFT): A heuristic technique for adding fault tolerance to an embedded device at the task graph level.

4) Scheduling: A technique for evaluating the current device architecture for performance.

5) Add Processing Element: A heuristic technique for adding an additional processing element (CPU or ASIC) to the current device architecture.

6) Synthesize Communication Links: A technique for arranging the system's processing elements into a hypercube topology and synthesizing all connecting communication links.

The first phase of the approach is concerned with defining the device requirements. Although any specification language can be used, the experimentation presented in this thesis has been specified in $C$ language. The second phase or the algorithm involves the profiling of the device specification. The specification is manually converted into task graph form. Each task in the task graph represents a functional section of the overall device. Each of these functional sections are timed for execution on each type of processor available in the library. Additionally hardware alternatives to the software implementation are developed and are profiled for both performance and area utilization. The profiling stage is complete once the software 
and hardware timing data and the hardware area data have been collected for each task in the task graph.

The third phase is the group based fault tolerance (GBFT) method which is applied to the input task graph. This heuristic was developed to add a minimal fault detection mechanism to the system and to simplify fault recovery. The algorithm adds additional assertion and duplicate/compare tasks to the task graph. It minimizes the fault detection overhead by exploiting a task's error transparency and combining tasks into groups. The quantity and type of spare PEs in the final device is set by the user. If a fault is detected by one of the added tasks, an additional processing element is signaled to commence execution of the failed task group. This simple heuristic provides a low overhead method for performing node-fault detection and recovery.

The fourth phase is the scheduling tec"inique (see the "Scheduling" block in Figure 3.1). This heuristic method was developed to efficiently evaluate the current device architecture to determine if it meets performance requirements. In order to improve device throughput, the scheduling technique utilizes the established RECOD retiming heuristic to support pipelining of the task graph [7]. The scheduler accurately predicts overall device performance by scheduling tasks based on data dependencies. If the scheduler finds a task execution configuration that allows the current device architecture to meet performance requirements then the co-synthesis algorithm terminates successfully. If the scheduler is unable to schedule the task graph within device performance constraints the co-synthesis algorithm proceeds to the fifth phase. 
The fifth phase is concerned with the addition of another processing element to the system (see the "Add Processing Element" block in Figure 3.1). This section of the co-synthesis algorithm analyzes the current device architecture, the current task mappings, each task's hardware/software performance data and each task's hardware area data to determine the ideal type of processing element to add to the current system. If this phase is successful in adding a processing element to the system, the co-synthesis algorithm proceeds to phase six. If this phase is unsuccessful in adding another processing element (unable to add more hardware while still meeting the device hardware area constraint) the co-synthesis algorithm terminates unsuccessfully and provides the user with the partial solution generated.

The sixth phase is concerned with arranging the system's processing elements within a hypercube configuration and synthesizing all of the communication links (see the "Synthesize Communication Links" block in Figure 3.1). This phase arranges all of the processing elements within a hypercube topology while attempting to keep PEs with high levels of intercommunication within close proximity of each other. Once all of the system's PEs have been arranged, communication links connecting the PEs are synthesized. Once this phase has completed, the co-synthesis algorithm proceeds to the scheduling phase. 


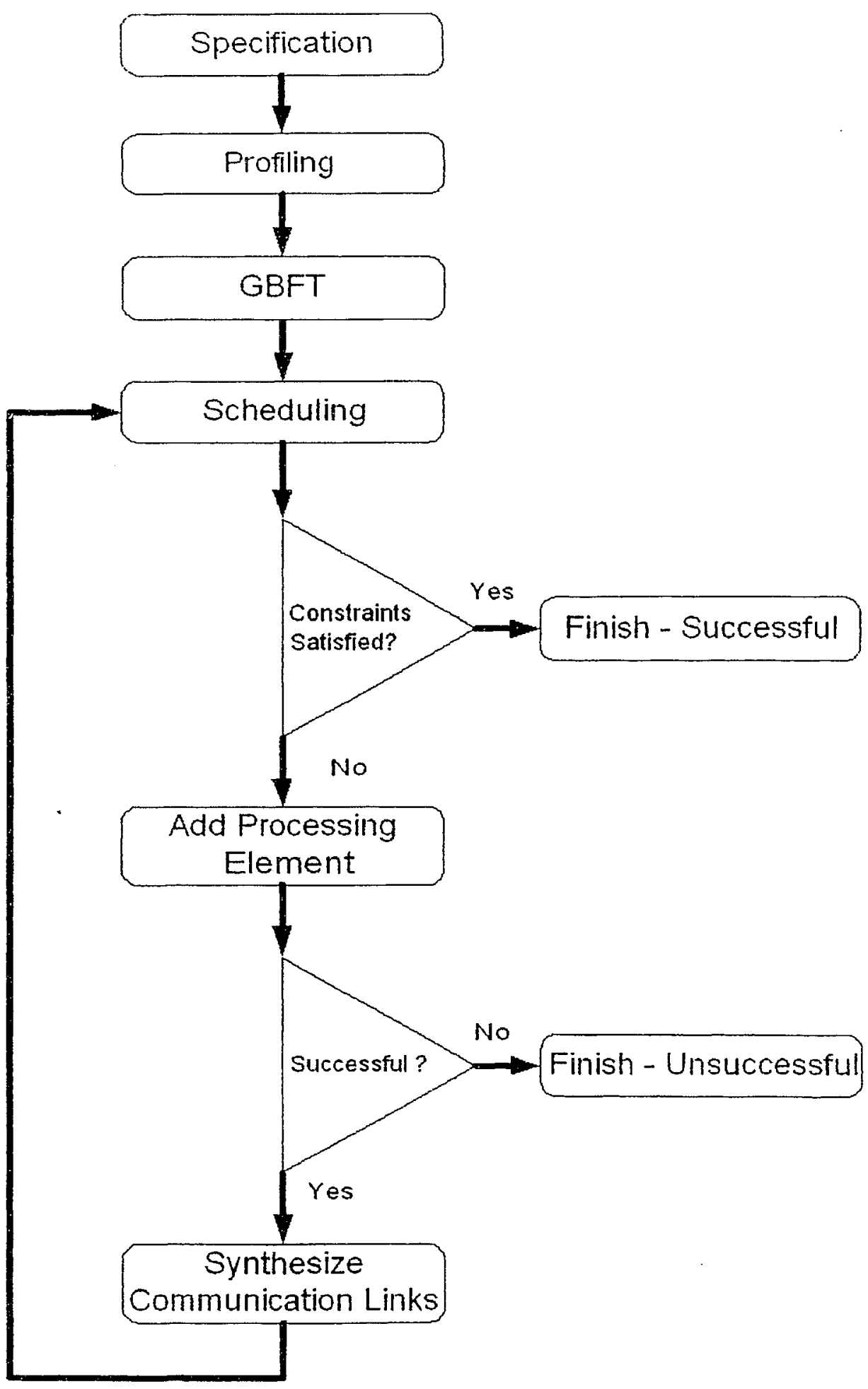

Figure 3.1: Hypercube Co-Synthesis Algorithm Flow 


\subsection{Fault Tolerance at the Task Graph Level}

\subsubsection{Overview}

This subsection discusses the two main preexisting techniques for adding fault detection/tolerance at the task graph level. Additionally, section 3.2 .4 presents an original contribution to task graph based fault tolerance techniques. In all cases fault detection is accomplished through the addition of fault detection tasks to the task graph. There are three types of tasks that any of the approaches presented may add to a task grapin: assertion tasks, duplicate tasks and compare tasks. An assertion task will analyze another task's output in order to determine whether the generated results are erroneous. A duplicate task reproduces the same work as another task in the graph. Typically a duplicate task will utilize an alternative implementation to that used by the task it is duplicating. A compare task will examine the results of two tasks to detect any inconsistencies. The addition of an assertion task typically requires considerably less computational overhead than the addition of a duplicate and compare set. However, assertion tasks are not always feasible. For an assertion task to be used, error states must be able to be detected by analyzing the results. An example assertion task could be the analysis of a checksum or checking that the generated results lie within an expected range. Duplicate and compare tasks tend to require a much higher computational overhead. First the entire task's functionality needs to be duplicated and then both generated results need to be compared. 


\subsubsection{Task Based Fault Tolerance}

Task based fault tolerance (TBFT) was developed by Yajnik et al. [49]. Task based fault tolerance is a technique designed to add fault detection capabilities at the task graph level. In task based fault tolerance, some form of error detection must be performed for the results generated by each node in the task graph. Due to the significant difference in fault tolerant overhead, assertion tasks are favoured in the task based fault tolerance algorithm. If a given task is capable of supporting assertions then an assertion task is added. Duplicate and compare tasks are only added if assertions are unavailable for the given task. To demonstrate these concepts Figure 3.2 provides an example input task graph. Figure 3.2 also illustrates the resultant task graph after processing by the task based fault tolerance algorithm. Here all of the tasks in the graph support the use of assertion tasks for error detection with the exception of task T3. Task T3 has had duplicate and compare tasks added to provide support for fault detection. 


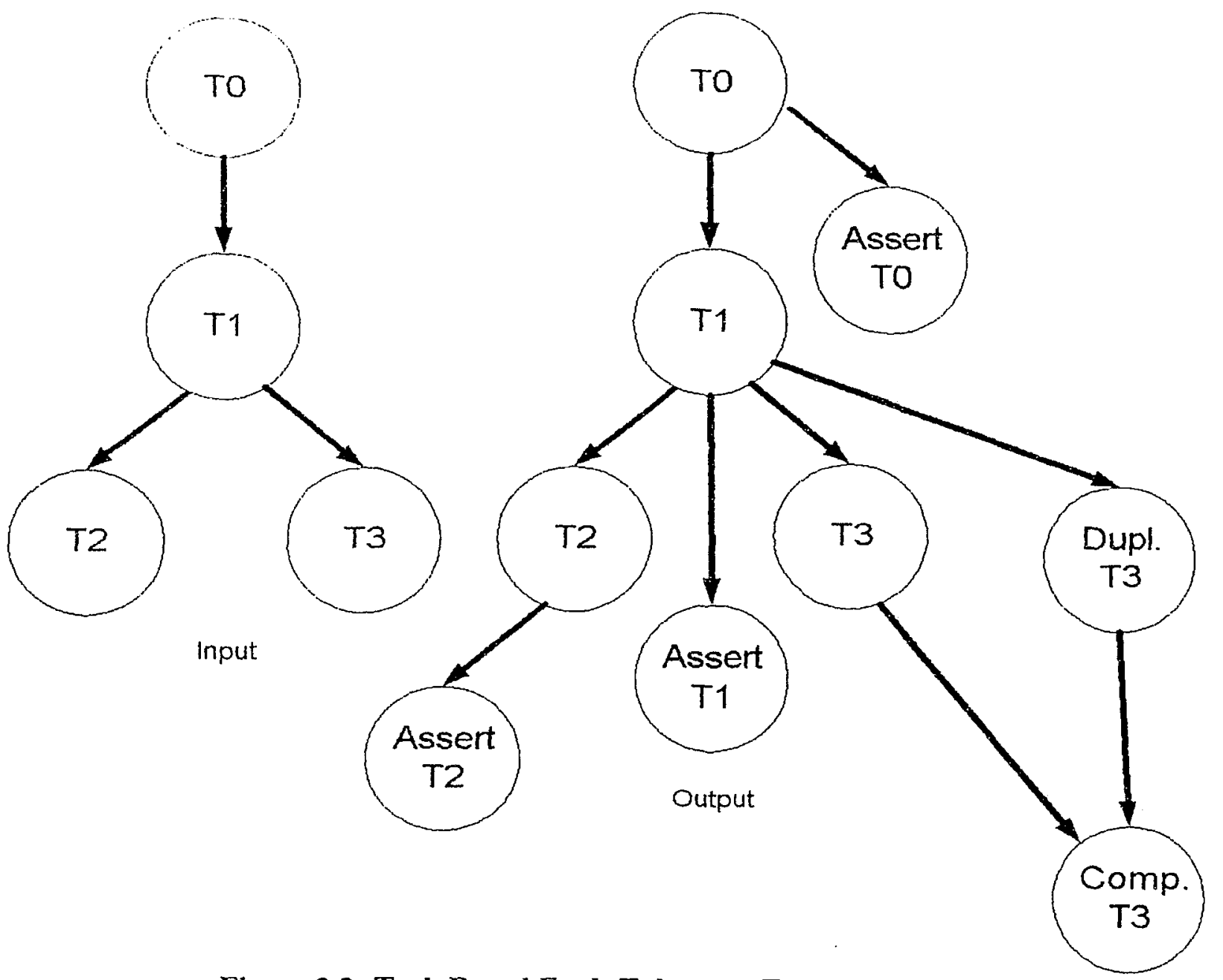

Figure 3.2: Task Based Fault Tolerance Example

\subsubsection{Cluster Based Fault Tolerance}

Cluster based fault tolerance was developed by Dave and Jha [13]. This technique was developed as an extension of the ideas presented in the task based fault tolerance algorithm. Modifications were made to the approach in order to reduce the substantial fault tolerant overhead prevalent in task based fault tolerance. In cluster 
based fault tolerance, Dave and Jha have introduced the concept of error transparency [13]. If a task provided with an erroneous input always produces an erroneous output then that task is said to be error transparent. This effect is exploited in their algorithm by grouping error transparent tasks into clusters which only require one assertion or duplicate/compare task. Figure 3.3 shows an example task graph input and the results generated by one iteration of the cluster based fault tolerance algorithm. Figure 3.4 shows the results after both 2 and 3 iterations of the algorithm. Figure 3.5 shows the final clusters for the given input task graph. After all of the tasks have been grouped into clusters, each cluster is given an assertion or duplicate/compare task to perform error detection. Figure 3.6 shows the final clustered task graph with the addition of error detecting assertion tasks. Each cluster is now treated as a single task in order to ensure that all tasks within a cluster are executed on the same processing element. In the example graph provided, all of the tasks are assumed to be error transparent.

The CBFT algorithm traverses a task graph based on task priority levels that favour tasks that are higher in the graph. The algorithm only allows one of a given task's children to be added to that task's cluster. Cluster based fault tolerance also introduces the concept of a maximum tolerated error detection time. If a large number of tasks were grouped into one cluster and an error occurs in the uppermost task, the error state would not be detected until all tasks in that cluster have completed execution. This may be undesirable as it will adversely affect performance. To avoid this problem, Dave and Jha have incorporated a user specified maximum tolerated error detection time into the cluster based fault tolerance algorithm [13]. The 
algorithm will not group more tasks into one cluster if the sum of the software execution times of those tasks exceeds the user specified maximum error detection time. In the cluster based fault tolerance algorithm, if an error is detected on a given cluster, the entire functionality of that cluster is moved to a spare processing element and signaled to recommence execution.
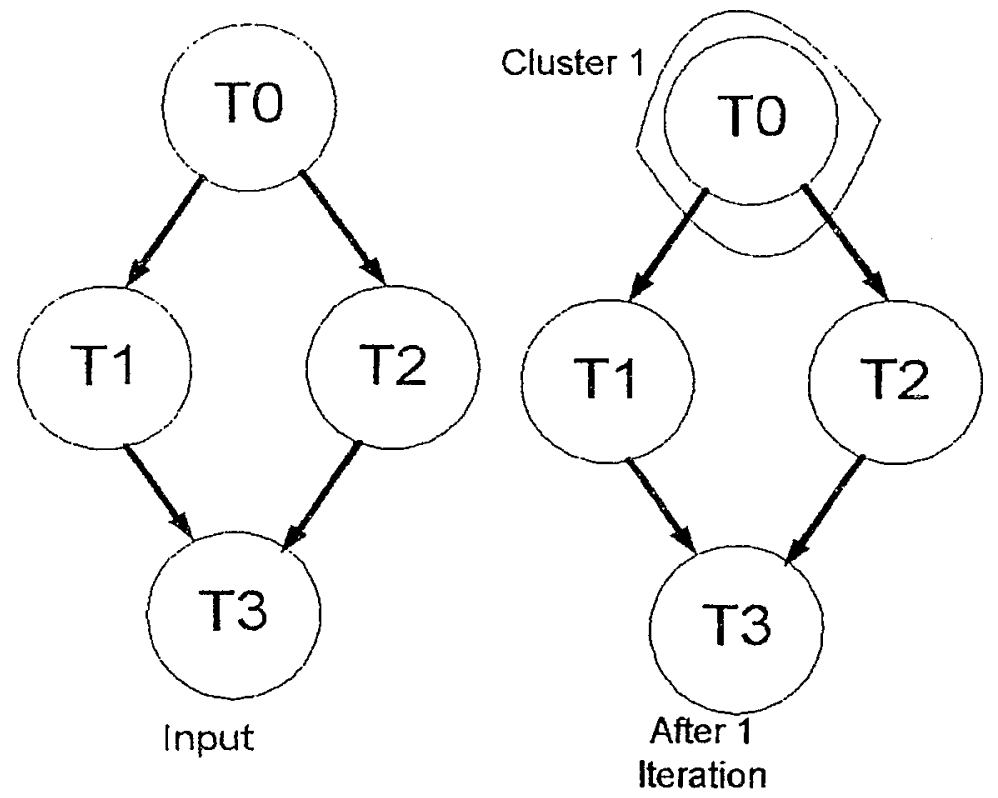

Figure 3.3: Cluster Based Fault Tolerance-Input \& 1 Iteration 


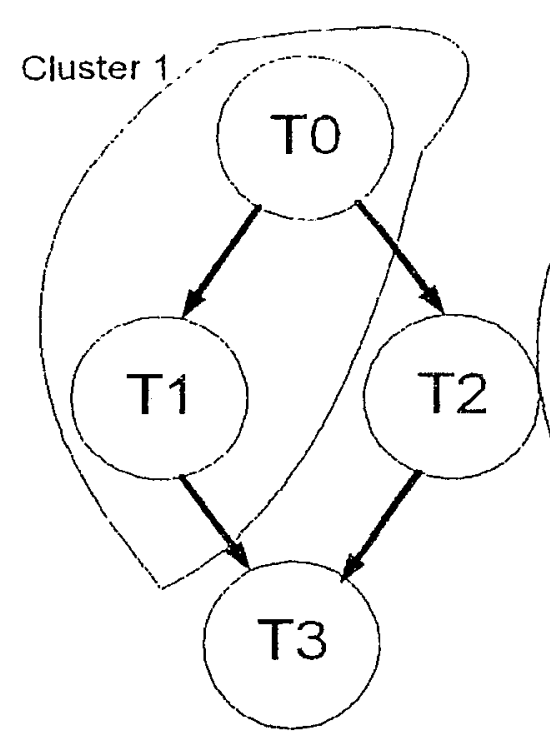

After 2 Iterations

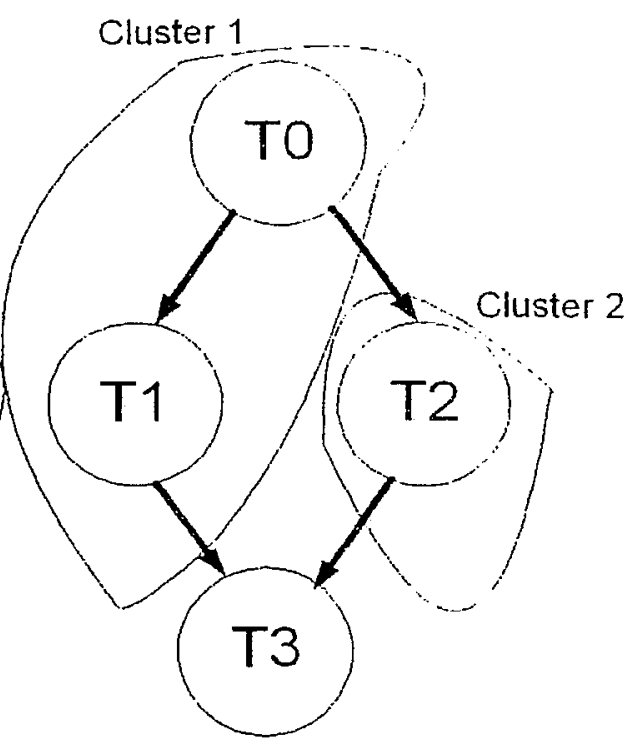

After 3 Iterations

Figure 3.4: Cluster Based Fault Tolerance -2 and 3 Iterations

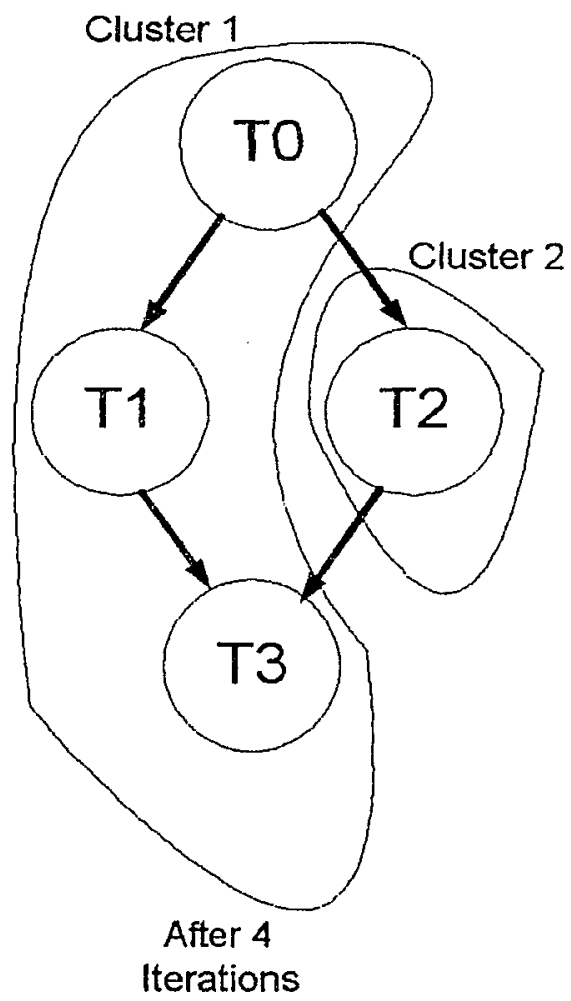

Figure 3.5: Cluster Based Fault Tolerance - Final Clustering 


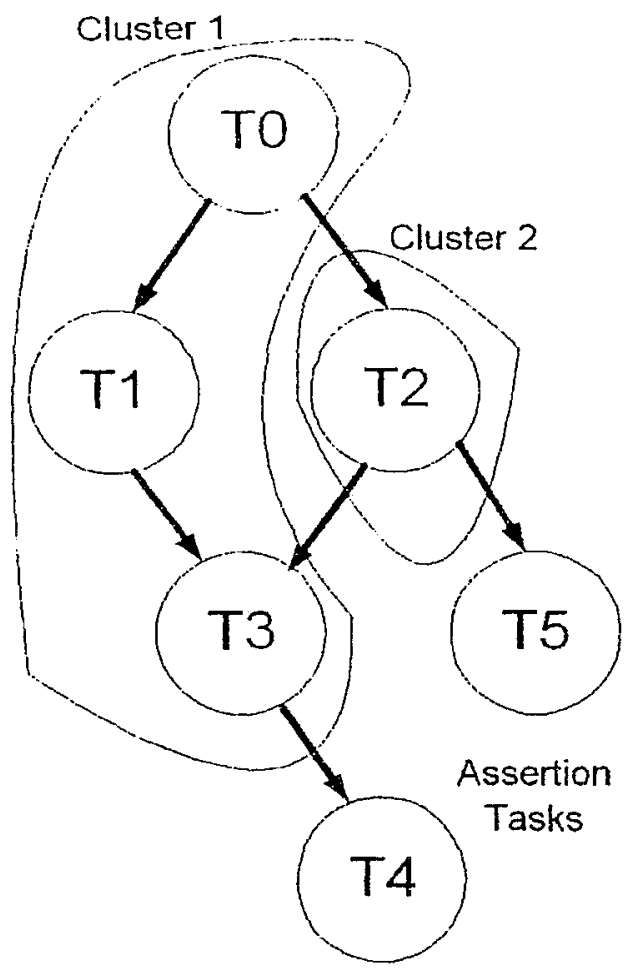

Figure 3.6: Final Added Assertion Tasks After Clustering

\subsubsection{Group Based Fault Tolerance}

This section presents a new and original technique for adding fault detection and tolerance at the task graph level named group based fault tolerance (GBFT). This technique was developed as an extension of the ideas presented in both the task based fault tolerance [49] and cluster based fault tolerance algorithms [13]. Modifications were made to the approaches in order to reduce the fault tolerant overhead prevalent. This algorithm utilizes the concept of error transparency. Group based fault tolerance also uses the concept of a user specified maximum tolerated error detection time. 
In group hased fault tolerance, the task graph is traversed from the lowest nodes to the highest nodes. Each leaf node (bottom level node) is assigned to its own group. Each of the leaf's parents are analyzed to determine whether they can potentially be added to the given leaf's group. A parent is considered a possibility for grouping in its child's group if it is not already grouped and if adding it to its child's group will not violate the user imposed error detection timing constraint. Once the set of parents eligible for grouping have been assembled for a given task, the parents are iteratively added to the group in order of decreasing fault tolerant overhead. This process is ended if the addition of another parent task to the group will violate the user defined error detection constraint. If a task has no children, its fault tolerant overhead is set to its assertion overhead. If a task $i$ does have children, its fault tolerant overhead is calculated to be:

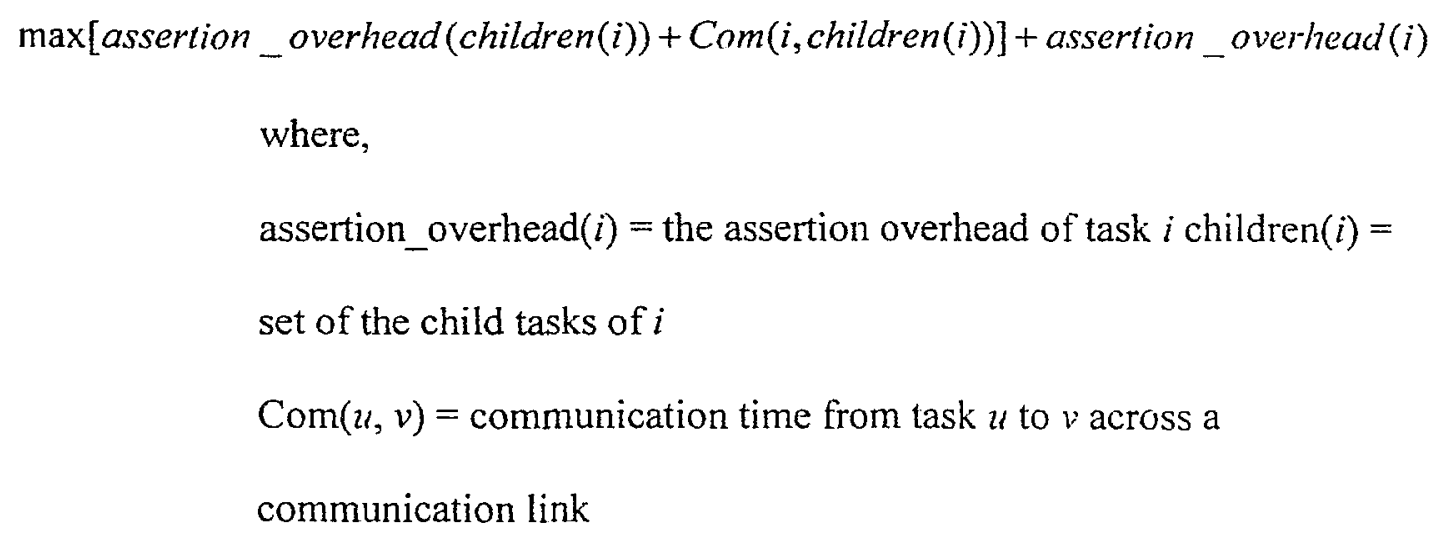

Figure 3.7 shows an example task graph input and the results generated by one iteration of the group based fault tolerance algorithm. Figure 3.8 shows the results after both 2 and 3 iterations of the algorithm. Figure 3.9 shows the final groupings for the given input task graph. Finally, Figure 3.10 shows the final clustered task 
graph with the addition of crror detecting assertion tasks. A more complex example of GBFT based task groupings is provided in Figures 4.6. 4.7, 4.8 and 4.9 in section 4.2. After all of the tasks have been grouped, each group is given an assertion or duplicate/compare task to perform error detection. Each group is now treated as a single task in order to ensure that all tasks within a group are executed on the same processing element. In the example graph provided, all of the tasks are assumed to be error transparent. In the group based fault tolerance algorithm, if an error is detected on a given cluster, the entire functionality of that cluster is moved to a spare processing element and signaled to recommence execution.

The group based fault tolerance algorithm adds assertion and duplicate/compare tasks to a task graph. It does not add assertion or duplicate/compare tasks to perform checks on the fault detection tasks that it adds. If fault detection of faults occurring in the GBFT added tasks is wanted, then it must be added manually after the GBFT algorithm has completed execution. 


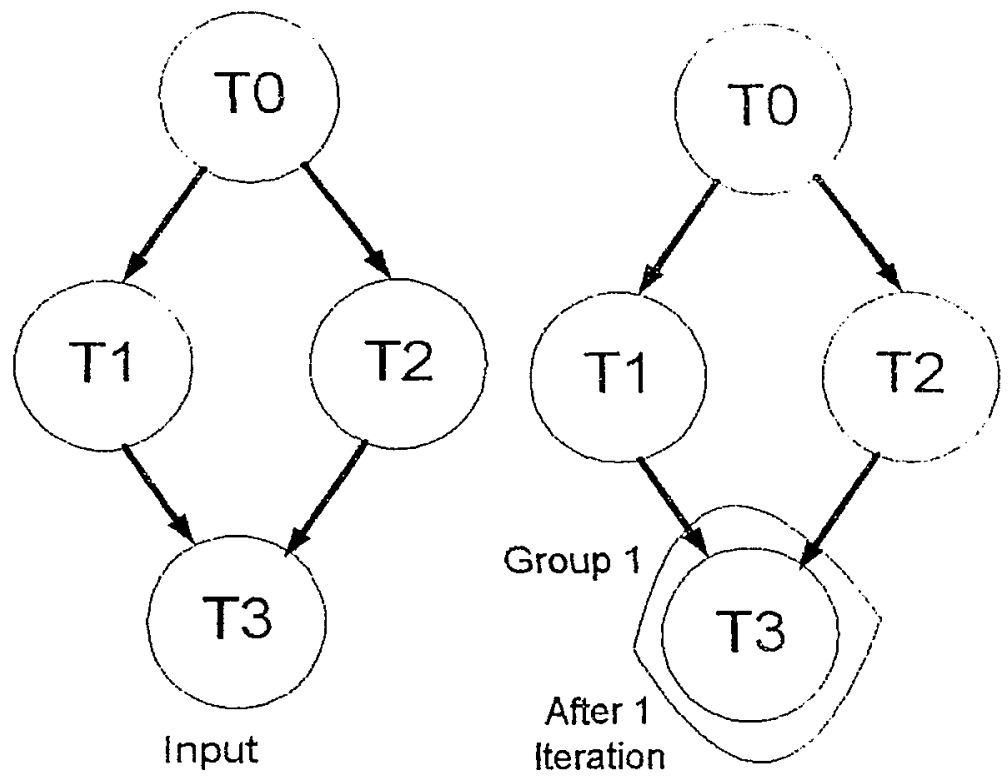

Figure 3.7: Group Based Fault Tolerance - Input \& 1 Iteration

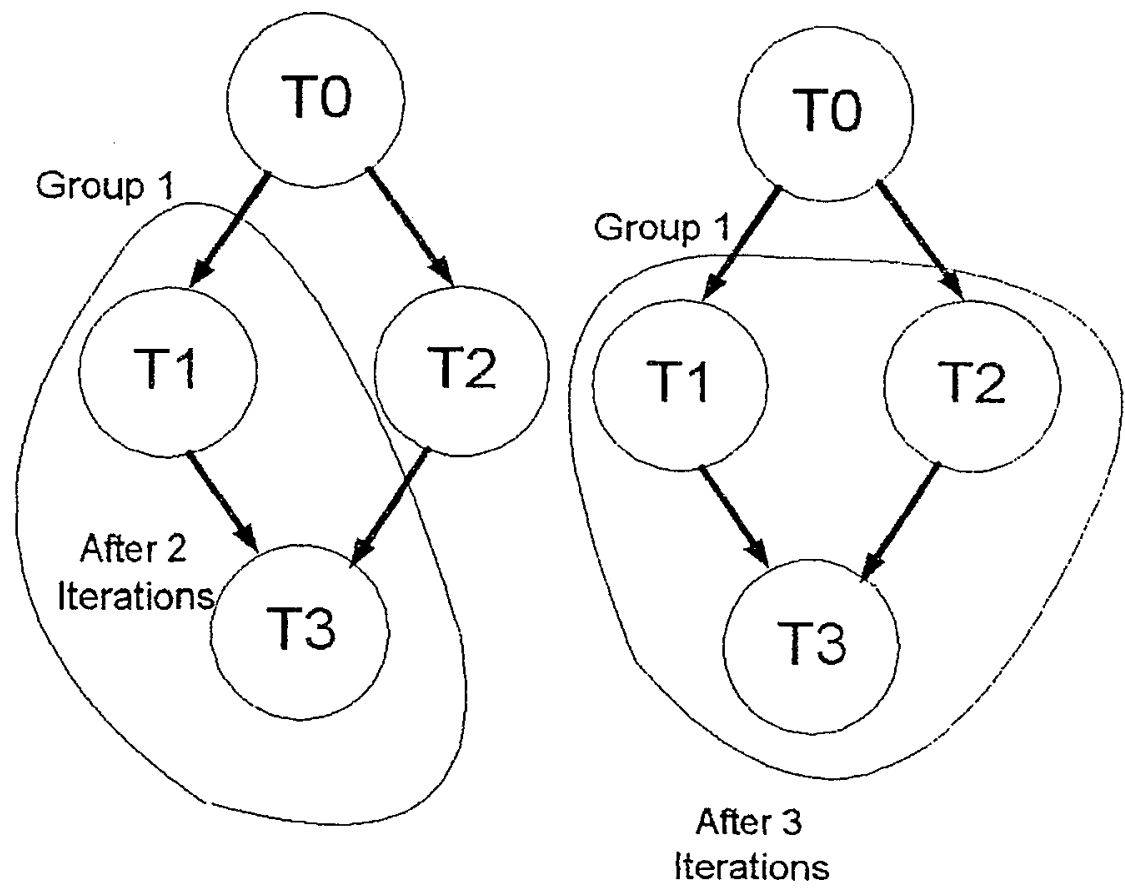

Figure 3.8: Group Based Fault Tolerance -2 \& 3 Iterations 


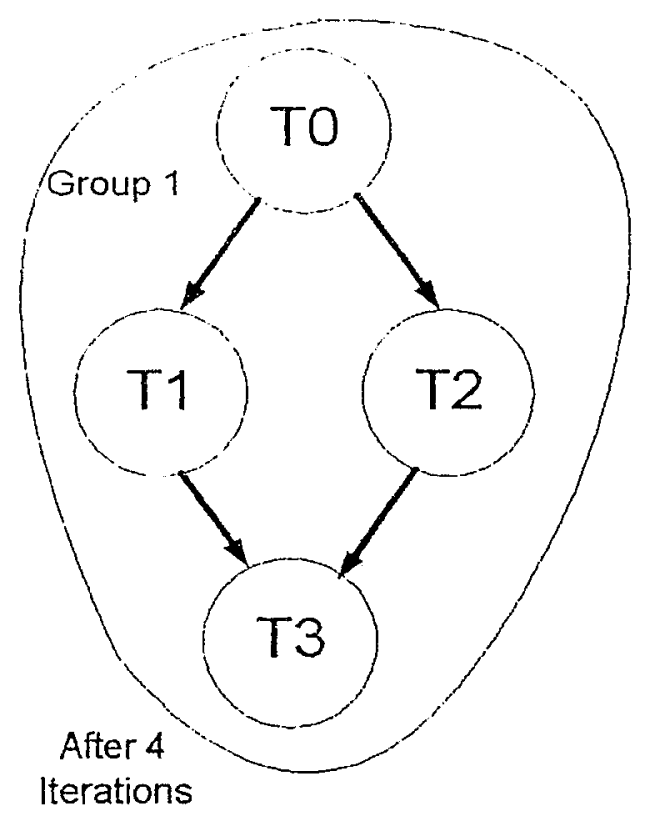

Figure 3.9: Group Based Fault Tolerance-Final Grouping

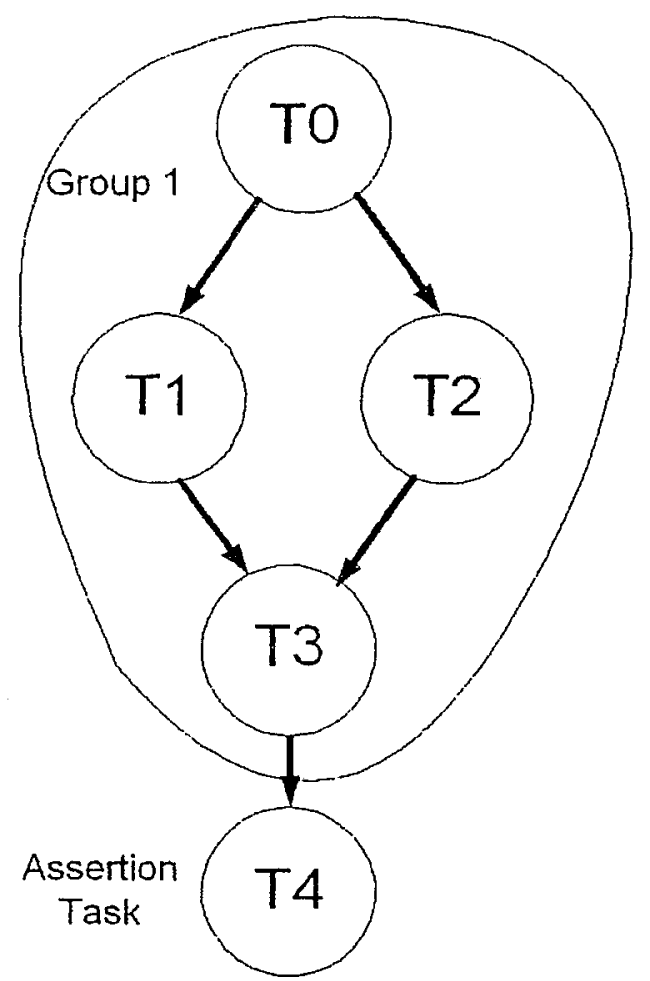

Figure 3.10: Final Added Assertion Task After Grouping 


\subsubsection{Task Graph Based Fault Tolerance Comparison}

A comparison of the results of the group based fault tolerance algorithm with both the cluster based and task based techniques is provided in order to evaluate the newly developed GBFT technique. Thirteen random test cases were generated and the output of all three algorithms is compared. The test cases have varying task graph configurations and varying user defined tolerated error detection times. The outputted error detection information from each algorithm is compared. The data collected has been assembled in Table 3.1. Here the values under the GBFT, CBFT and TBFT columns are the counts of error detection tasks added to the task graph. The fewer number of error detection tasks added to a task graph results in a smaller fault tolerance overhead. A smaller fault tolerance overhead is desirable as it will yield a fault tolerant device that utilizes less hardware and/or less computation time.

The first randomly generated task graph has been provided in Figure 3.11. Task graph 4 corresponds to the task graph from the MPEG decoding case study. The task graph can be found in Figure 4.1. Task graph 5 corresponds to the task graph from the block matching case study and can be found in Figure 4.6.

The information gathered in the table reveals that the group based fault tolerance technique yields a $18.75 \%$ improvement in fault tolerance overhead over the cluster 
based fault tolerance technique and a $45.83 \%$ improvement over the task based fault tolerance tochnique.

Table 3.1: Fault Tolerance Comparison Data

\begin{tabular}{|c|c|c|c|c|}
\hline Task Graph \# & $\begin{array}{c}\text { Tolerated Error Detection Time } \\
(\mathrm{msec})\end{array}$ & GBFT & CBFT & TBFT \\
\hline 1 & 4 & 2 & 3 & 8 \\
\hline 1 & 3 & 3 & 3 & 8 \\
\hline 1 & 2 & 4 & 4 & 8 \\
\hline 2 & 3 & 7 & 7 & 11 \\
\hline 2 & 2 & 7 & 8 & 11 \\
\hline 3 & 9 & 1 & 3 & 9 \\
\hline 3 & 8 & 2 & 3 & 9 \\
\hline 3 & 7 & 2 & 3 & 9 \\
\hline 3 & 6 & 3 & 3 & 9 \\
\hline 3 & 5 & 3 & 3 & 9 \\
\hline 3 & 4 & 3 & 3 & 9 \\
\hline 3 & 3 & 4 & 3 & 9 \\
\hline 3 & 2 & 5 & 5 & 9 \\
\hline 4 & 800 & 1 & 16 & 22 \\
\hline 4 & 300 & 15 & 16 & 22 \\
\hline 4 & 120 & 14 & 17 & 22 \\
\hline 4 & 80 & 17 & 17 & 22 \\
\hline 4 & 40 & 21 & 21 & 22 \\
\hline 4 & 34 & 22 & 22 & 22 \\
\hline 5 & 150000 & 1 & 16 & 22 \\
\hline 5 & 40000 & 13 & 16 & 22 \\
\hline 5 & 30000 & 14 & 16 & 22 \\
\hline 5 & 20000 & 15 & 16 & 22 \\
\hline 5 & 15000 & 16 & 16 & 22 \\
\hline
\end{tabular}




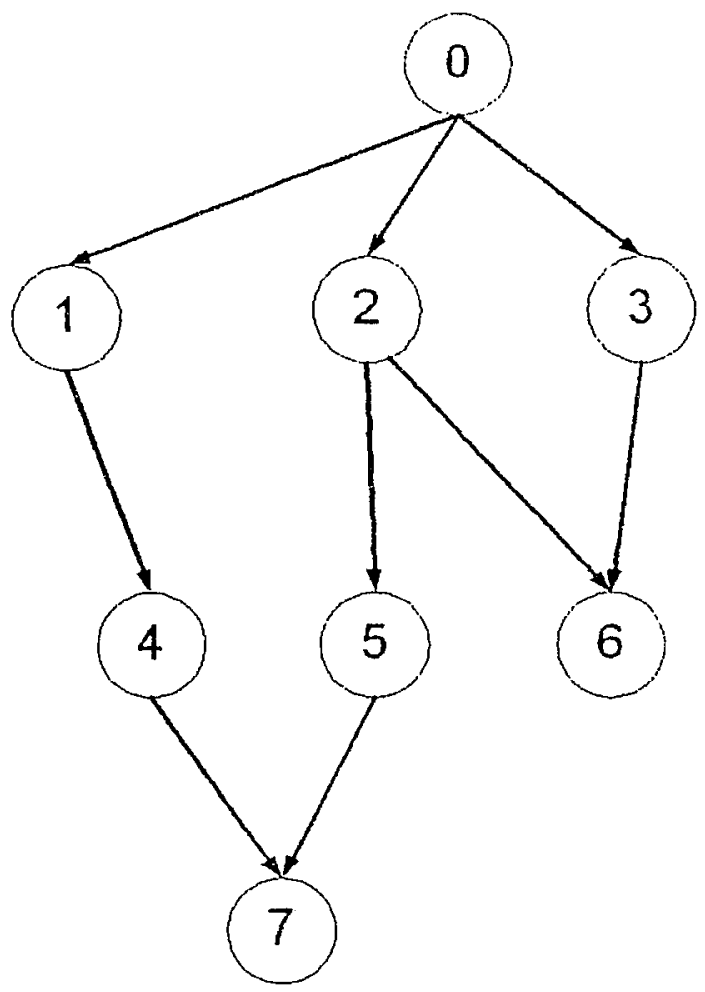

Figure 3.11: Randomly Generated Task Graph (Graph \#1)

\subsection{Pipelined Scheduler}

The functionality described in this sub-section occurs in the "Scheduling" block in Figure 3.1. The effectiveness of a given architecture alternative is evaluated by obtaining a pipelined schedule that is executed in the algorithm's scheduling phase. A pipelined schedule with a minimum period $P$ is an assignment of completion times of all tasks, $F(v)$, such that for all tasks $v$ in the system $0<=F(v)<:=P$. For a task $v$ 
with a data dependence $e=(u, v)$, where $u$ is a parent of $v$, the schedule time of $v$ must honour the following equation:

$$
\begin{array}{ll}
F(v) \geq F(u)+v_{\cdots+}+C(11, v) & \text { if } \operatorname{PSMatch}(u, v) \\
F(v) \geq r_{i w} & \text { otherwise }
\end{array}
$$

where,

$v_{e x e c}=$ execution time of task $v$

$C(u, v)=$ the overall communication time between mapped tasks $u$ and

$v(0$ if no communication)

$\operatorname{PSMatch}(u, v)=$ true if tasks $u$ and $v$ are located on the same pipelined stage, false otherwise

This definition requires that a task will not commence execution before receiving the required data from all parent tasks. The scheduler takes communication delays and resource usage into account when assigning tasks to processing elements. The algorithm utilizes the established RECOD retiming transformation to divide the task graph into multiple pipelined stages $[6,7]$.

The RECOD retiming transformation divides a task graph into multiple pipelined stages by inserting a cut-line which separates two tasks (parent and child) and defines the separation between two pipelined stages. The location of this cut-line is dependent on the parent and child's current pipeline stage, the parent and child's mapping, the length of the constraining path of the parent and the amount of information passed between the parent and child. 
Initially, the RECOD transformation is repeatedly used to divide the task graph into a maximum number of pipelined stages. After performing the RECOD retiming transformation, the scheduler attempts to assign completion times to all tasks to satisfy device performance requirements. If unsuccessful, the algorithm attempts to improve the task allocations iteratively in order to minimize device communication overhead. A flow diagram of the scheduler's operation is provided in Figure 3.12. 


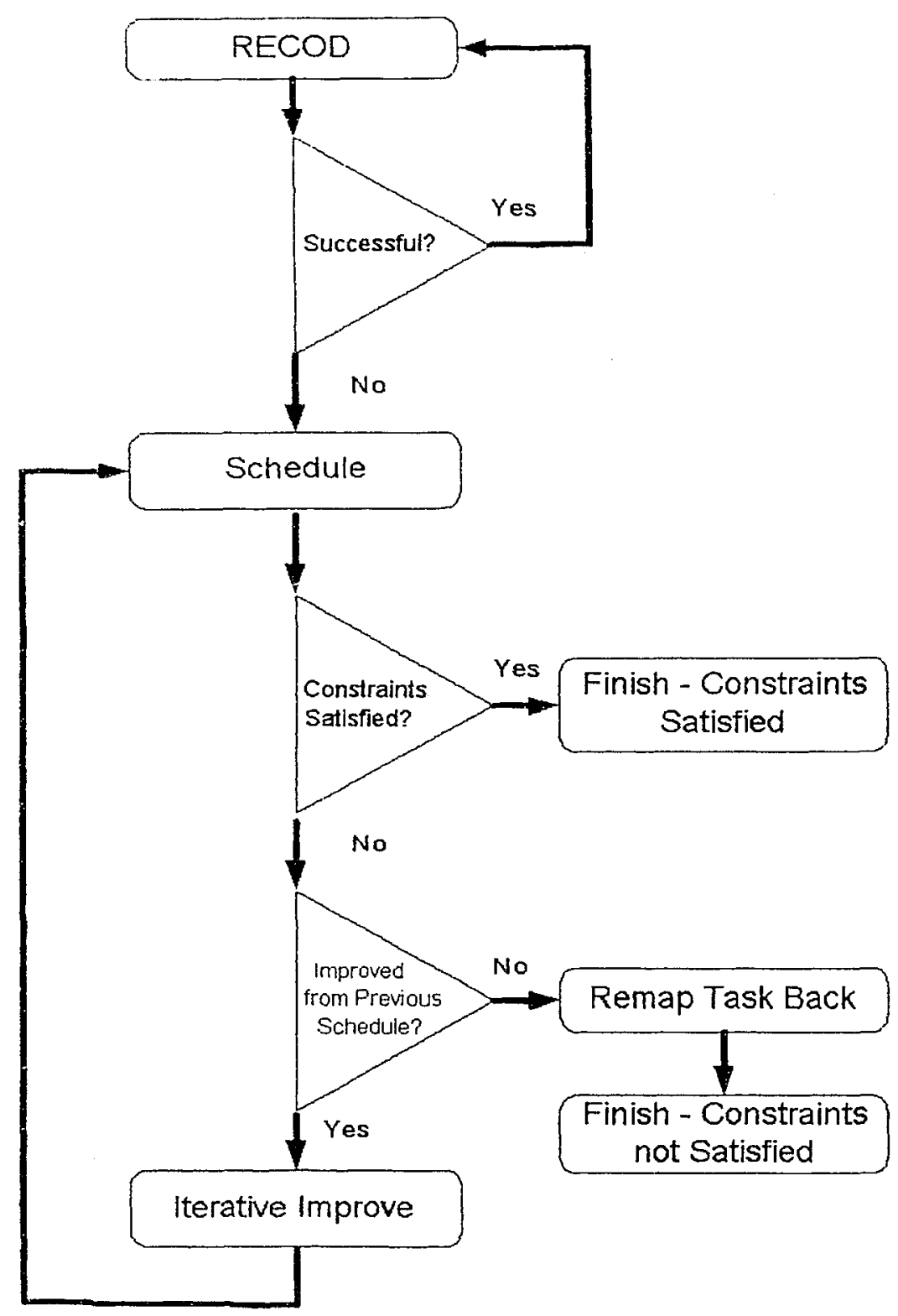

Figure 3.12: Hypercube Co-Synthesis Scheduler

For the purposes of scheduling ("Scheduling" block in Figure 3.1), each task from the input task graph is assigned to one of the three sets, $m, n$ and $p$. In order to specify the first set, pathLoadChild, pathLoadParent and pathLoad variable values are assigned to each task $v$. These values are defined as: 


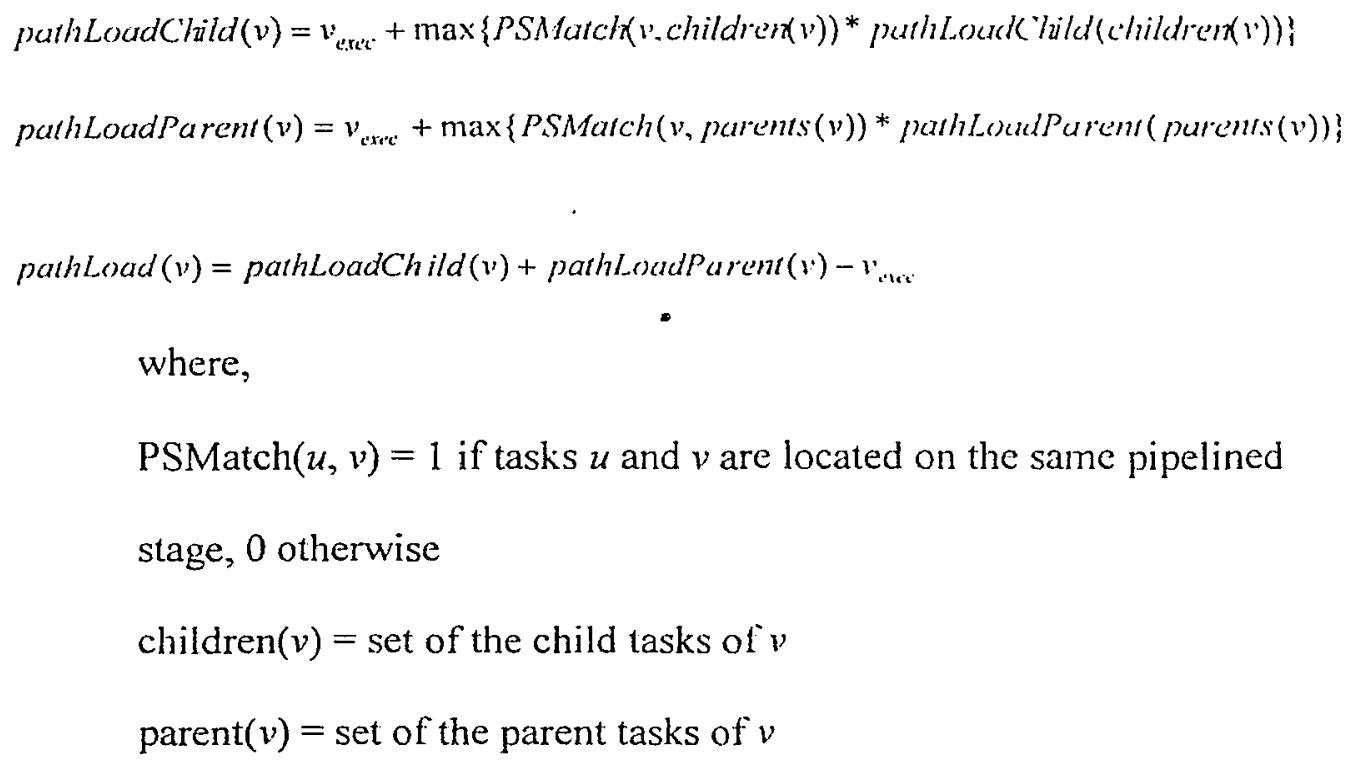

As is result, a task v's pathLoad is the value of the heaviest loaded path containing task $v$.

The first set of tasks $m$ is defined as:

$\exists m A T \rightarrow(\operatorname{pathLoad}(m)==\max \{\operatorname{pathLoad}(A T)\})$

where,

$\exists, \rightarrow$, and $==$ represent there exists, such that and equivalent

respectively

AT is the set of all tasks

Set $m$ is the set of tasks that are located on the constraining path and are scheduled first. Set $w^{\prime}$ consists of all the tasks having a path to $m$. The second set $n$, is defined as: $n=w-m$.

Set $n$ consists of tasks that are ancestors of all the tasks of set $m$ excluding the members of set $m$. 
The final set $p$, is defined as: $p=A T-m-n$. Set $p$ consists of the remaining ungrouped tasks and will be scheduled last.

These sets are used to prioritize the tasks for scheduling. The constraining path is the longest path through the graph in terms of the execution times of its tasks. When scheduling a task of set $m$ that has unscheduled parents, the parents are scheduled first. Dividing tasks into these set configurations prioritizes tasks located on the constraining path. The tasks on the constraining path are mist likely to adversely affect the target device performance and so the motivation is to schedule them first. If a system schedule is obtained that meets the performance requirements, the schedule function exits successfully, otherwise the scheduler attempts to improve iteratively. Excessive communications is a potential problem in hypercube topologies as they can slow down overall system performance by causing PEs to wait for data. When two tasks are located far from each other in the architecture, communication between them may require multiple hops. In order to alleviate this potential problem, the algorithm includes an iterative improver (as labeled in Figure 3.12) whose goal is to refine the task mapping. The value commLevel for each task is defined as:

$$
\begin{aligned}
& \text { commLevel }(v, P E)=\sum \operatorname{Com}(v, x)^{*} N h(v, x)+\sum \operatorname{Com}(u, v)^{*} N h(u, v) \\
& \text { where, } \\
& \operatorname{Com}(u, v)=\text { communication time from task } u \text { to } v \text { across a } \\
& \text { communication link } \\
& N(u, v)=\text { number of links (hops) that must be traversed to } \\
& \text { communicate between tasks } u \text { and } v
\end{aligned}
$$




$$
\begin{aligned}
& x=\text { children }(v) \\
& u=\text { parents }(v)
\end{aligned}
$$

A task $q$ is selected for ré-mapping based on the following equation:

$$
\begin{aligned}
& q=\arg \max \left[\frac{\operatorname{execTime}(v, \operatorname{execLoc}(v))+\operatorname{commLevel}(v, \operatorname{exec} L(v c(v))}{\operatorname{execTime}(v, A P E)+\operatorname{commLevel}(v, A P E)}\right] \\
& \text { where, } \\
& \text { exec } \operatorname{Loc}(v)=\text { the execution location (PE) of task } v \\
& \operatorname{exec} \operatorname{Time}\left(v, P E^{\prime}\right)=\text { the execution time of task } v \text { on } P E^{n} \\
& A P E=\text { the set of all processing elements }
\end{aligned}
$$

The above equation selects task $\mathrm{q}$ for mapping based on total performance gain. Task $q$ is subsequently remapped to a new processing element to improve the overall execution and communication times. This process is repeated as long as the scheduler reveals continued improvements in the overall solution. When this iterative process does not provide any further improvements, the scheduler exits unsuccessfully. This technique was incorporated in order to help minimize any unnecessary communications.

\subsection{Device Expansion}

The functionality described here is executed as part of the "Add Processing Element" block of the design flow given in Figure 3.1. This co-synthesis section is responsible 
for expanding the current system architecture to include an additional processing element (PE). The PE type can be a CPU or an application specific integrated circuit (ASIC). The primary focus of this function is to determine the most effective type of PE to be added. The addition of a PE should provide a maximum performance enhancement with a minimum additional hardware area. If the addition of a PE causes a violation of the hardware area constraint, the function exits unsuccessfully. Otherwise, the function will add a new PE and exit successfully. In order to make a knowledgeable decision on which $\mathrm{PE}$ to add, the algorithm estimates the ratio of expected performance improvement due to the increase in hardware area for all the available options. For these caiculations the variable, speedupSW is included as a preliminary estimate of performance improvement due to the addition of another CPU and defined as:

$$
\begin{aligned}
& \text { speedupSW }=T S W_{\text {exec }}(A T)^{*}(1-T P /(T P+1)) \\
& \text { where, } \\
& T S W_{\text {exec }}(v)=\text { the total execution time of all tasks mapped to SW from } \\
& \text { set } v \\
& T P=\text { the count of total CPUs in the current system architecture }
\end{aligned}
$$

The variable ratioSW is defined that estimates the performance to hardware area ratio for adding a CPU and is defined as:

$$
\begin{aligned}
& \text { ratios } W=E x L^{*} \text { speedup } S W / P A C \\
& \text { where, } \\
& \qquad P A C=\text { the area cost for the addition of another } \mathrm{CPU}
\end{aligned}
$$




$$
E x L=\text { the expected load of the new processor }
$$

ExL is provided to weight the decision of adding a new processor based on the expected load that processor would receive. ExL is defined as:

$$
\begin{array}{lc}
E x L=0 & \text { if } C S T<=T P \\
E x L=C S T /\left(k^{*} T P\right) & \text { if } C S T>T P \text { and } C S T<k^{*} T P \\
E x L=1 & \text { otherwise } \\
\text { where, } & C S T=\text { the count of tasks currently mapped to software }
\end{array}
$$

\begin{abstract}
As an estimation, it is assumed that if the current system architecture contains $k$ times as many tasks mapped to software as there are CPUs, then the newly added processor will be provided with a full task load. The value $k$ is user set. These equations were selected to quickly approximate the expected amount of speedup obtained by adding an additional CPU to the system.
\end{abstract}

With respect to the speedup and ratio factors for adding new ASICs, a task set $v$ is declared as the set of all tasks currently mapped to software. The speedupHW[v] and ratioHW $[v]$ array variables are then calculated for the set of tasks in $v$. Each individual ratioHW value represents an expected improvement factor for moving the given task from its current location in software to an ASIC implementation. The speedupHW[v] represents the difference between the execution time of task $v$ in software and in hardware. In order to define ratioHW the variables $I$ CouldBen and 
IWillBen are created. The $t$ CouldBen[v] variable is defined as the count of lasks having an identical hardware configuration pattern as task $v$. The tWillBen[v] variable is defined as the count of tasks having an identical hardware configuration pattern as task $v$ and is currently mapped to software. The ratioHW[v] is defined as:

$$
\begin{aligned}
& \text { ratioHW }[v]=\frac{\text { speedupHW[v] }}{A(v)}+\left(1-\frac{\text { speedupHW }[v]}{A(v)}\right) *\left(\frac{t \text { WillBen }[v]-1}{t \operatorname{CouldBen}[v]}\right) \\
& \text { where, } \\
& A(v)=\text { the area cost for the hardware implementation of task } v
\end{aligned}
$$

The above formulation encourages the selection of hardware solutions that can be reused by multiple tasks. All of the ratio values (both hardware and software) obtained are compared and the maximum value is used to decide the PE type to be employed in the expansion. When the PE being added is an ASIC ( $P E_{A S / C}$ ), the function assigns a maximum number of tasks with matching configuration patterns to the newly added ASIC, given that the following constraints are met:

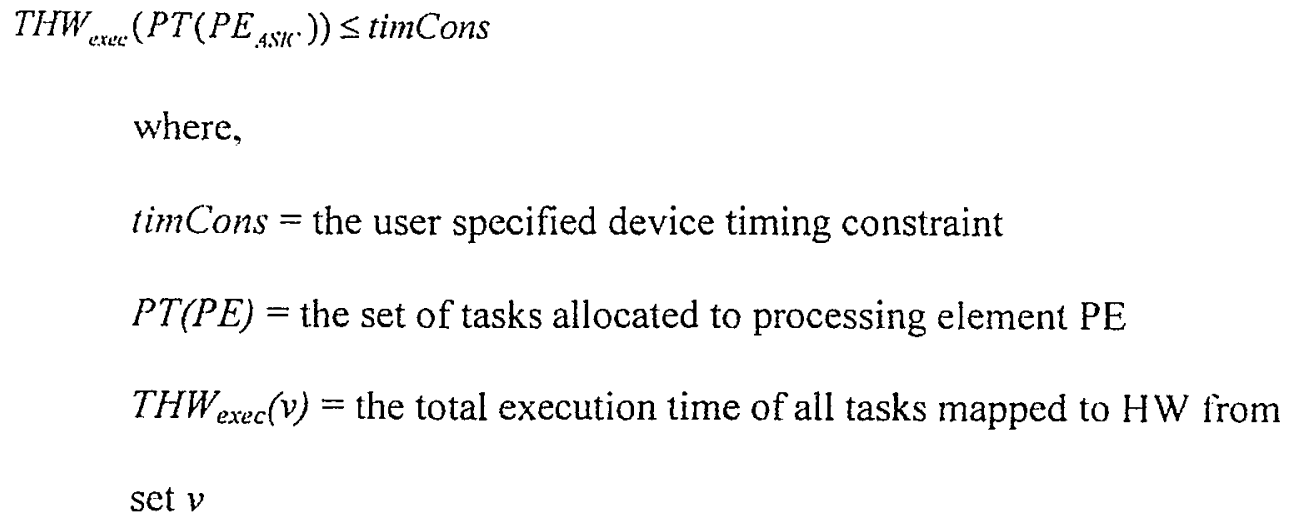


When the added PE is a CPU, the function must decide which tasks will be initially allocated to the new CPU. The task to be allocated to the new processor $P_{m}$. is selected from the processor identified by the variable $P_{\text {donor }}$ in the following equation:

$$
\begin{aligned}
P_{d \text { dimar }}= & \max \left(T S W_{\text {cxecc }}(P T(A P))\right) \\
& \text { where, } \\
& A P \text { is the set of all processors }
\end{aligned}
$$

The above equation ensures that the donor processor would always be the most heavily loaded. The task with the lowest communication overhead with respect to the other locally allocated tasks is selected for remapping from the donor process. This is intended to minimize the communication overhead and simplify the iterative process for the scheduler. After this task has been reallocated to the new PE, the selection of a donor processor and a task is repeated until the new CPU load reaches the average processor load. To accomplish this, the variable loadAverage $=T S W_{c a c} / T P$ is defined. where $T P$ is the total number of processors. This process is repeated until an allocation is obtained such that the difference between the load on the new processor and the average load is a minimum.

While adding new hardware, the algorithm tracks the overall area of the device to ensure an accurate prediction of the final system cost. The hardware area overhead associated with each communication interface located at each PE node is recorded, in addition to the cost of each communication link. 


\subsection{Communication Link Integration}

The functionality described here is executed in the "Synthesize Communication Links" block of the design flow provided in Figure 3.1. This section of the cosynthesis algorithm is responsible for connecting all the system PEs and assignment of communication links to form a hypercube topology. To limit the communication overhead. the processing elements with high levels of inter-communication are placed as close as possible in a hypercube topology. An array of communication coefficients is defined, com Coeff $[T P E]$ (where TPE = the count of total processing elements), for each processing element. The comm Coeff for processing element $P E_{n}$ with respect to $P E_{m}$ is defined as:

$$
\begin{aligned}
& P E A r r a\left[P E_{n}\right] \cdot \operatorname{com} \operatorname{Coeff}\left[P E_{n t}\right]=\sum \operatorname{Com}\left(\operatorname{PET}\left(P E_{n}\right), \operatorname{PET}\left(P E_{m}\right)\right) \\
& \text { where, } \\
& P E T(P E)=\text { the set of tasks currently allocated to } P E \\
& P E A r r a y=\text { the array of all processing elements }
\end{aligned}
$$

In order to clarify the process of assigning specific locations to each processing element within the framework of a hypercube architecture, the established binary naming convention for labeling nodes is utilized [29]. The binary naming convention in a $3 \mathrm{D}$ hypercube is shown in Figure 3.13. This naming convention is convenient for message routing and the number of communication hops between two nodes can be 
quickly determined by the number of bit-wise mismatches between the binary identifiers for each node.

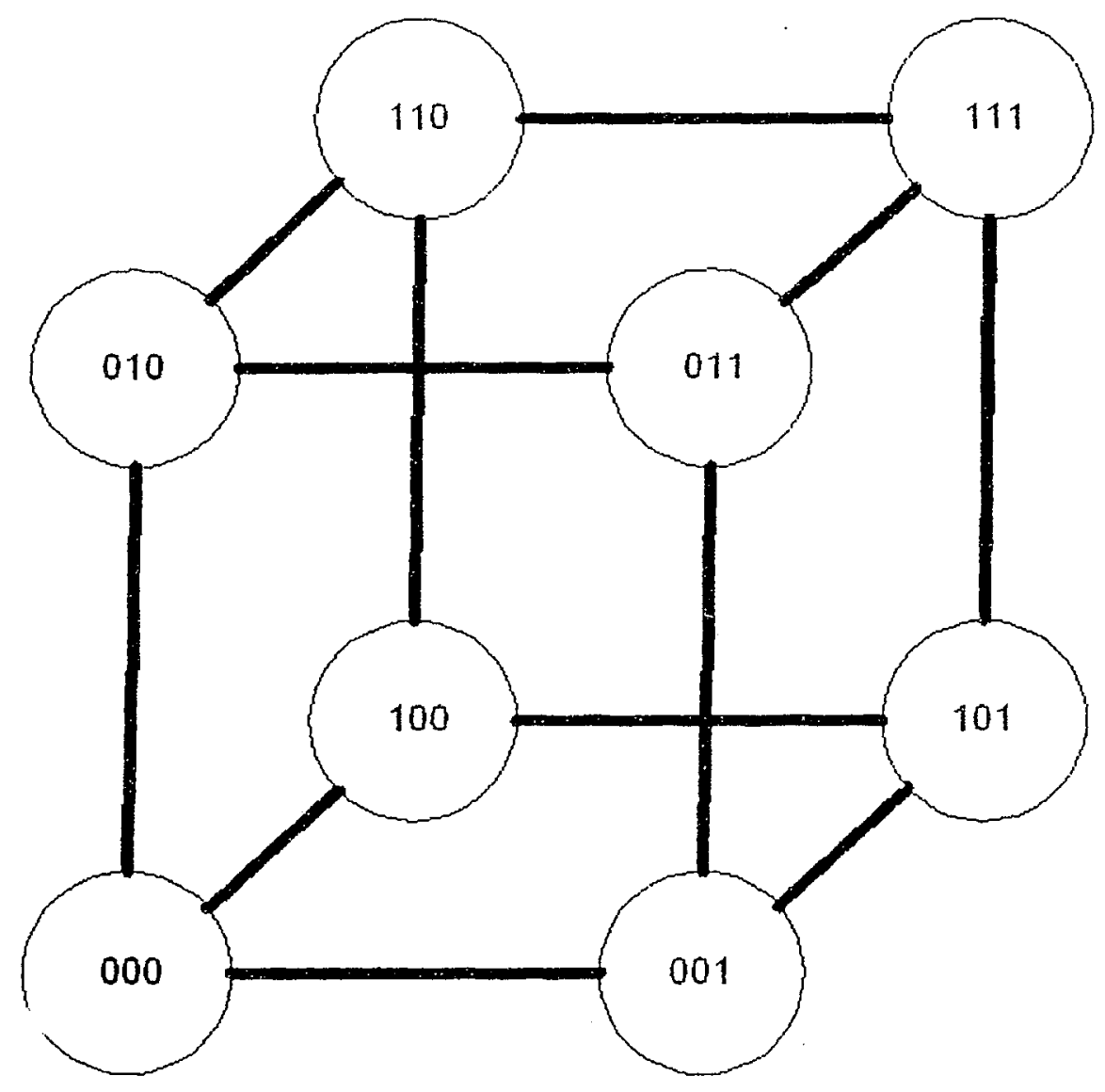

Figure 3.13: Binary Naming for Hypercube Nodes

Initially, the first CPU is placed at location 000. Subsequently, each location (hN) is filled by the PE named in the variable selectedPE by the following definitions:

$\exists p e$ in $A P E \rightarrow h e(h N, B L(p e))$

selectedPE $=\arg \max \left(\sum^{n \in P \cdot} P E A r r u y[n] . c o m(\operatorname{coc} f f[U P E])\right.$

where, 
$A P E=$ the set of all processing elements currently assigned to a specific hypercube location

$B L(p e)=$ the binary number that processing element pe is assigned to $h e(h N, h M)=$ the hypercube edge connecting binary location $h N$ to binary location $h M$ $U P E=$ the set of all processing elements currently unassigned to a specific hypercube location

The next PE to place in the hypercube is selected from the set of unassigned PEs. The PE is selected such that it has the largest communication coefficient with respect to the already assigned PEs that will be its neighbour. This process is repeated until all the PEs are allocated to hypercube nodes. This heuristic was selected to minimize the occurrence of long communication delays.

The co-synthesis algorithm does not require the hypercube architecture to be perfectly symmetrical. If all the processing elements are not a power of two, some of the PEs will have fewer communication links than the others. This is generally considered undesirable as one of the advantages of hypercube architectures lies in its innately fault-tolerant topology. Consequently, if the total number of processing elements is not a power of two, an additional communication link to compensate for the shortage in the most recently added PE is introduced. This communication link connects the $P E$ in the highest binary location (finalPE), with a destinationPE. In order to determine the destinationPE, the set of processing elements. neigh is defined as the 
set of PEs that share a neighbour with the finalJE. The processing element destination $P E$, is selected to be the task identified by:

$\max (P E A$ rray[finalPE].comCoeff $[n]) \quad$ while $n \in$ neigh

The above equation was selected to connect the final PE with the compatible PE with which it communicates most often. To illustrate this example consider Figure 3.14, where a 5-node hypercube is provided and the final communication link will connect the PE at node (100) to the shaded PE with which it communicates most. As previously mentioned, one of the advantages of hypercube topologies is its inherent fault tolerant capabilities. The motivation for the above heuristic is to eliminate the potential catastrophic failure that could occur if the communication link connecting node (000) and (100) fails. This heuristic preserves fault tolerance in nonsymmetrical hypercuhe systems. 


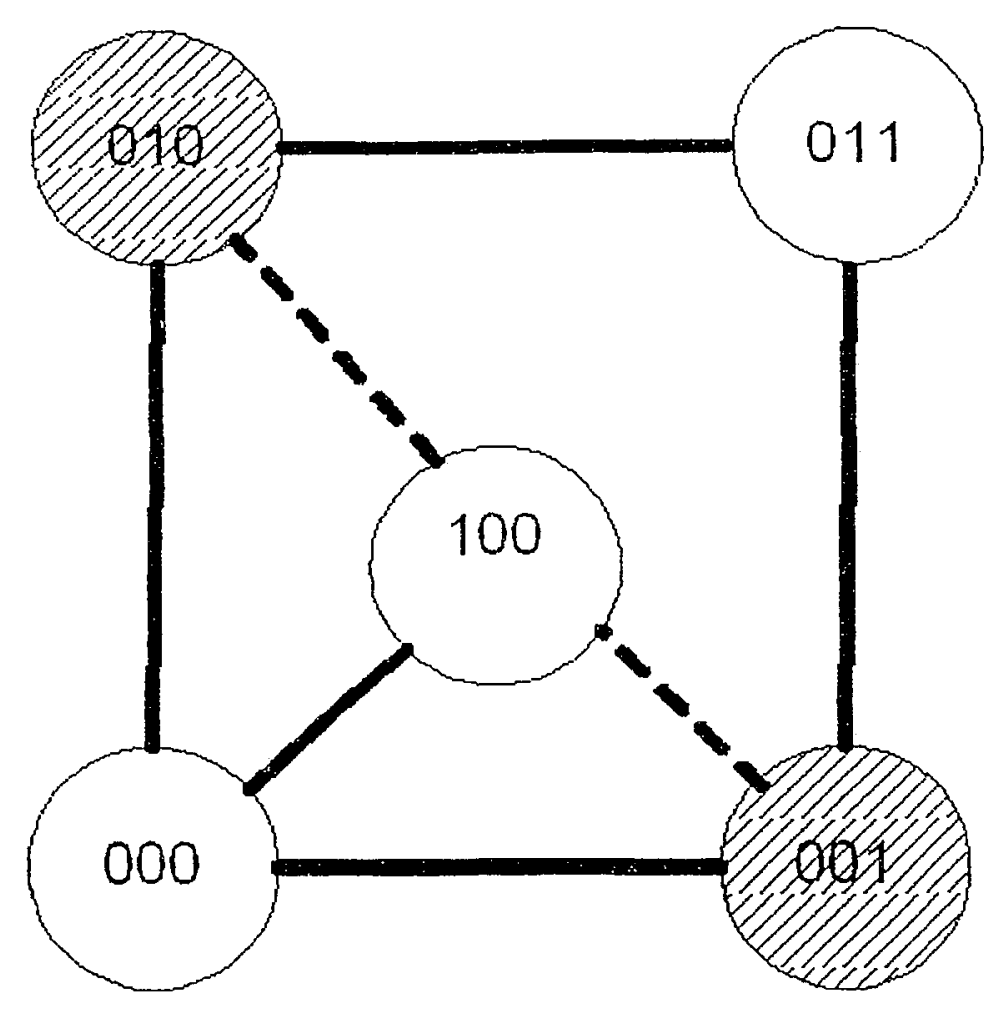

Figure 3.14: Non-Symmetrical 3-D Hypercube

In summary the approach consists of six phases. The first three are performed once. and the final three are repeatedly executed until a final device architecture has been generated. The first phase is responsible for clearly defining the device's functional and non-functional requirements. The second phase is concerned with converting the functional requirements into task graph form and gathering all of the relevant data needed in the later phases. The third phase adds fault detection tasks to the task graph, thus facilitating low overhead fault tolerance. The fourth phase involves evaluating the device's performance and determining if it meets non-functional performance requirements. The fifth phase is concerned with adding a processing 
element to the system. Finally, the sixth phase is responsible for synthesizing all of the device's communication links. 


\section{CHAPTER 4}

\section{IMPLEMENTATION AND EXPERIMENTAL RESULTS}

\subsection{Parallel MPEG-2 Decoding}

An effective demonstration of the capabilities of the co-synthesis algorithm would have to involve an application that is computationally intensive. Additionally, for demonstrative purposes it is beneficial to implement a device that is easily understood. A multiple PE embedded device was implemented, which is responsible for decoding 16 MPEG-2 video streams and simultaneously comparing their decoded images to predefined data. Such a device could be used in autonomous navigation or security systems. The device was fully specified in C language encompassing 9500 lines of code. A task graph representation was obtained by analysis of the specification and is provided in Figure 4.1. The functionality of the system is expressed as a graph of 22 tasks. The design space of the application has been tested in terms of device area and timing constraints in order to evaluate the performance of the presented approach. The test and experimental results are provided in Figure 4.2. The results of an optimal technique that exhaustively attempts each possible combination is also included. This is useful for demonstrating the quality of the algorithm and its results as compared to the optimal solution. 


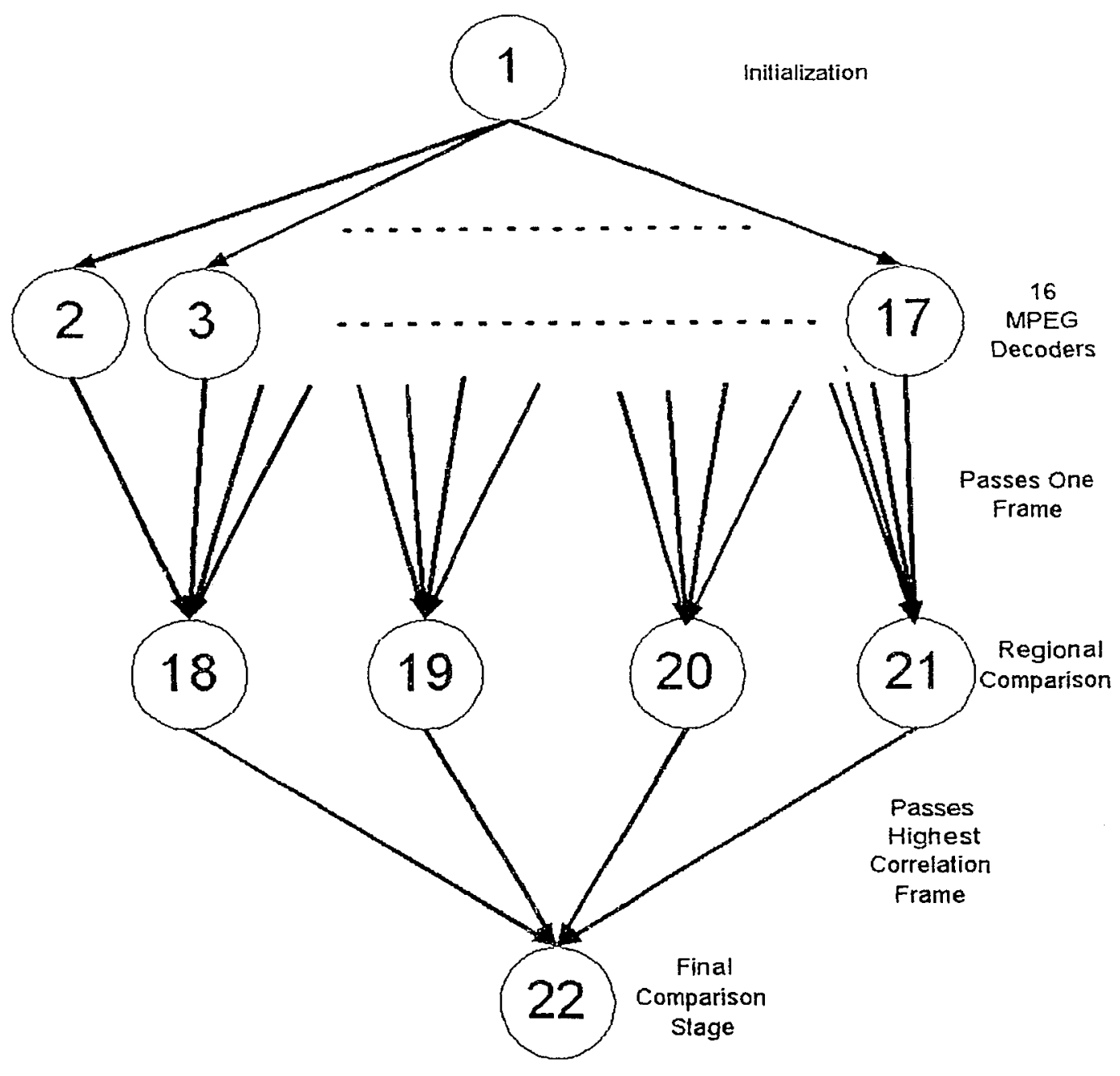

Figure 4.1: Parallel MPEG Decoding: Functional Task Graph

A set of hardware and software implementation data is provided to the algorithm. In order to establish the software execution times for each task, the $\mathrm{C}$ language based specification was profiled on a Pentium II $450 \mathrm{MHz}$ CPU. The algorithm library can consist of many CPU types however, for simplicity and practicality only the Intel Pentium II $450 \mathrm{MHz}$ CPU was included for this case study. Each of the tasks were fully implemented in hardware and profiled for performance and hardware area requirements on an Altera FLEX10KE FPGA. The only exception is the MPEG-2 
decoding tasks, where the pre-existing IP core from Amphion Semiconductor was utilized for the hardware implementation. Table 4.1 provides information regarding the quantity of each type of PE used for each of the design constraints studied.

The co-synthesis algorithm provides support for fault tolerant communications by generating the target device into a hypercube architecture. By using the group-based fault tolerance techniques (GBFT) previously outlined, a fault tolerant embedded device has been synthesized. The GBFT algorithm adds assertion and duplicatc/compare tasks to the task graph. Upon detection of a failure, the assertion/compare task signals one of the spare PEs to commence execution of the failed task. The type and number of spare PEs are defined by the user. It should be noted that in order to compare the algorithm with the optimal case the GBFT section of the algorithm had to be disabled. The GBFT algorithm would substantially increase the total number of tasks in the task graph. The optimal approach is extremely computationally intensive and unable to generate results for larger task graphs within a reasonable amount of time. Although node fault tolerant versions of this case study have been simulated, the data presented illustrates results obtained without the use of the GBFT portion of the algorithm. Further analysis that includes the error detection tasks added by the GBFT portion of the algorithm is provided in section 4.2 . 


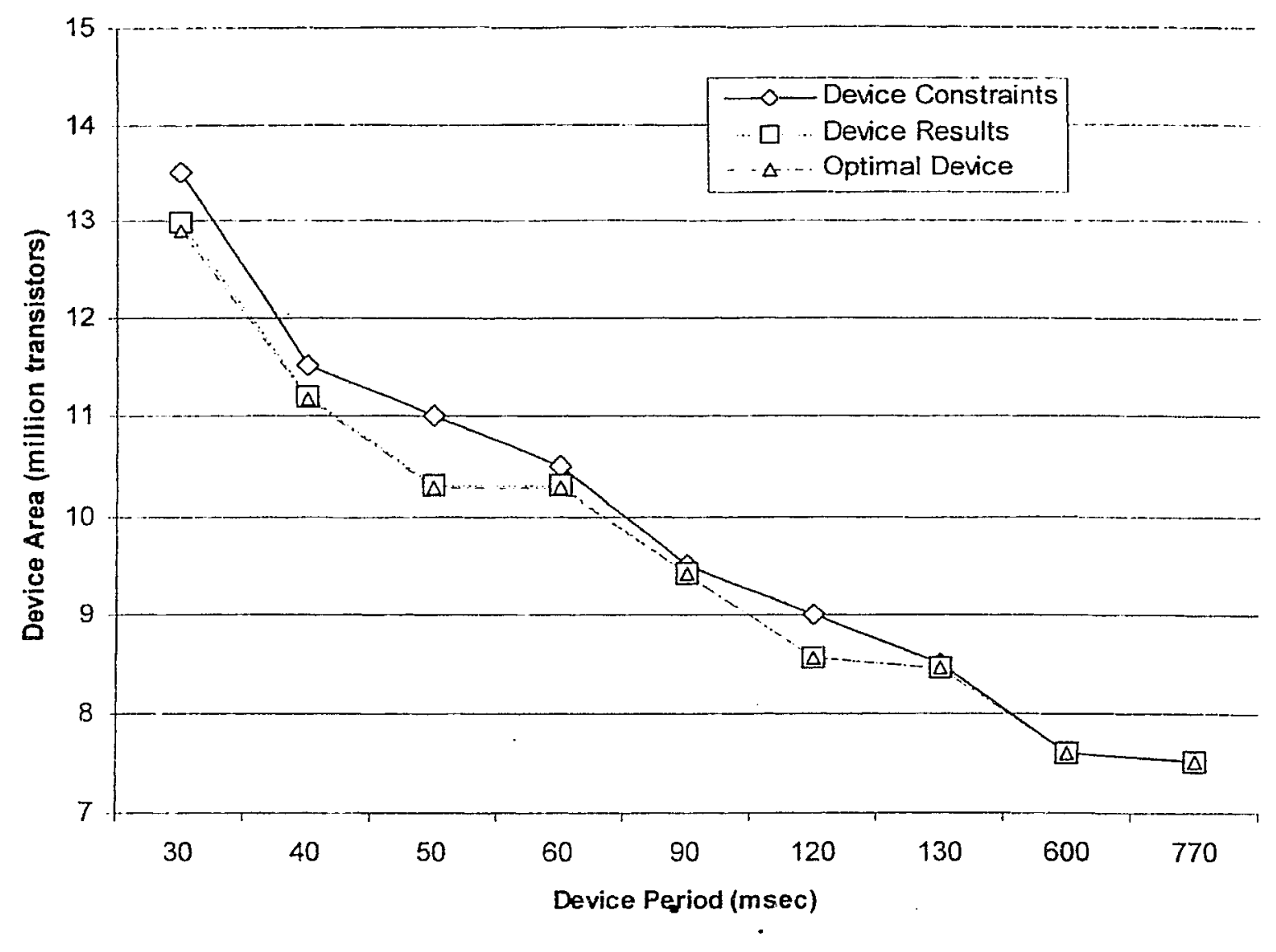

Figure 4.2: Design Space Exploration of Parallel MPEG Decoding

The results provided in Figure 4.2 demonstrate the ability of the method to synthesize embedded devices with varying design constraints. A comparison of hardware area to performance trade-offs can be very useful to an embedded engineer in order to examine differences between design alternatives. The results from using an exhaustive (optimal) mapping technique is also provided. The exhaustive approach attempts all the possible combinations and is very computationally intensive. Comparison results from the co-synthesis algorithm with those obtained by the optimal technique revealed that the algorithm produces embedded devices with $96.25 \%$ of the performance obtained by the optimal technique on average. 
Additionally, the embedded devices generated by the algorithm only utilized $0.62 \%$

(average) more hardware than the devices obtained by the optimal method.

Table 4.1: Processing Element Utilization of Design Space for MPEG Decoding

\begin{tabular}{|c|c|c|c|c|c|c|c|c|}
\hline $\begin{array}{c}\text { Area } \\
\text { Constraint } \\
\text { (millions of } \\
\text { transistors) }\end{array}$ & $\begin{array}{c}\text { Timing } \\
\text { Constraint } \\
\text { (msec) }\end{array}$ & $\begin{array}{l}\text { Initial } \\
\text { HW - } \\
\text { Task } 1\end{array}$ & $\begin{array}{c}\text { MPEG - } \\
\text { Decoding } \\
\text { Cores } \\
\text { Tasks 2-17 }\end{array}$ & $\begin{array}{l}\text { HW-Reg. } \\
\text { Comparison } \\
\text { Tasks 18-21 }\end{array}$ & $\begin{array}{c}\text { HW - Final } \\
\text { Comparison } \\
\text { Task } 22\end{array}$ & $\begin{array}{l}\text { Total } \\
\text { PEs }\end{array}$ & $\begin{array}{c}\text { Exec. } \\
\text { Time } \\
\text { (msec) }\end{array}$ & 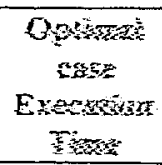 \\
\hline 13.5 & 30 & 1 & 6 & 1 & 1 & 10 & 35.2 & $-24 \sin$ \\
\hline 11.5 & 40 & 0 & 4 & 1 & 1 & 7 & 14.8 & $-\frac{1}{4} 6 \pi$ \\
\hline 11 & 50 & 0 & 3 & 1 & 1 & 6 & 11.2 & 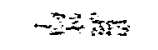 \\
\hline 10.5 & 60 & 0 & 3 & 1 & 1 & 6 & 13.5 & 等解 \\
\hline 9.5 & 90 & 0 & 2 & 1 & 1 & 5 & 7.9 & - zetis \\
\hline 9 & 120 & 0 & 1 & 1 & 1 & 4 & 4.2 & $-2+4$ \\
\hline 8.5 & 130 & 0 & 1 & I & 0 & 3 & 3.4 & $-\mathrm{ins}$ \\
\hline 7.6 & 600 & 0 & 0 & 1 & 0 & 2 & 1.6 & Ams \\
\hline 7.5 & 770 & 0 & 0 & 0 & 0 & 1 & 0.8 & - \\
\hline
\end{tabular}

Table 4.1 provides detailed information regarding the configuration of each solutisti that the co-synthesis algorithm produced. Given the design constraints provided is the first two columns, the algorithm generated a device that could meet specifications. The next four columns (3-6) provide the count of various ASIC circuits present in the final synthesized system. The total PEs column provides a count of the total number of PEs present in the given device. The count of CPUS in the table have not been included as there is only one CPU for each case. The execution time column stowe 
the length of time the algorithm took to execute on a Pentium IV in order to synthesize the device. The final column provides the approximate execution times from performing exhaustive co-synthesis. Figure 4.3 provides the final device architecture for the first test case provided in table 4.1. Figure 4.4 provides the final device architecture for the second test case. The architecture for the fifth test case is provided in Figure 4.5.

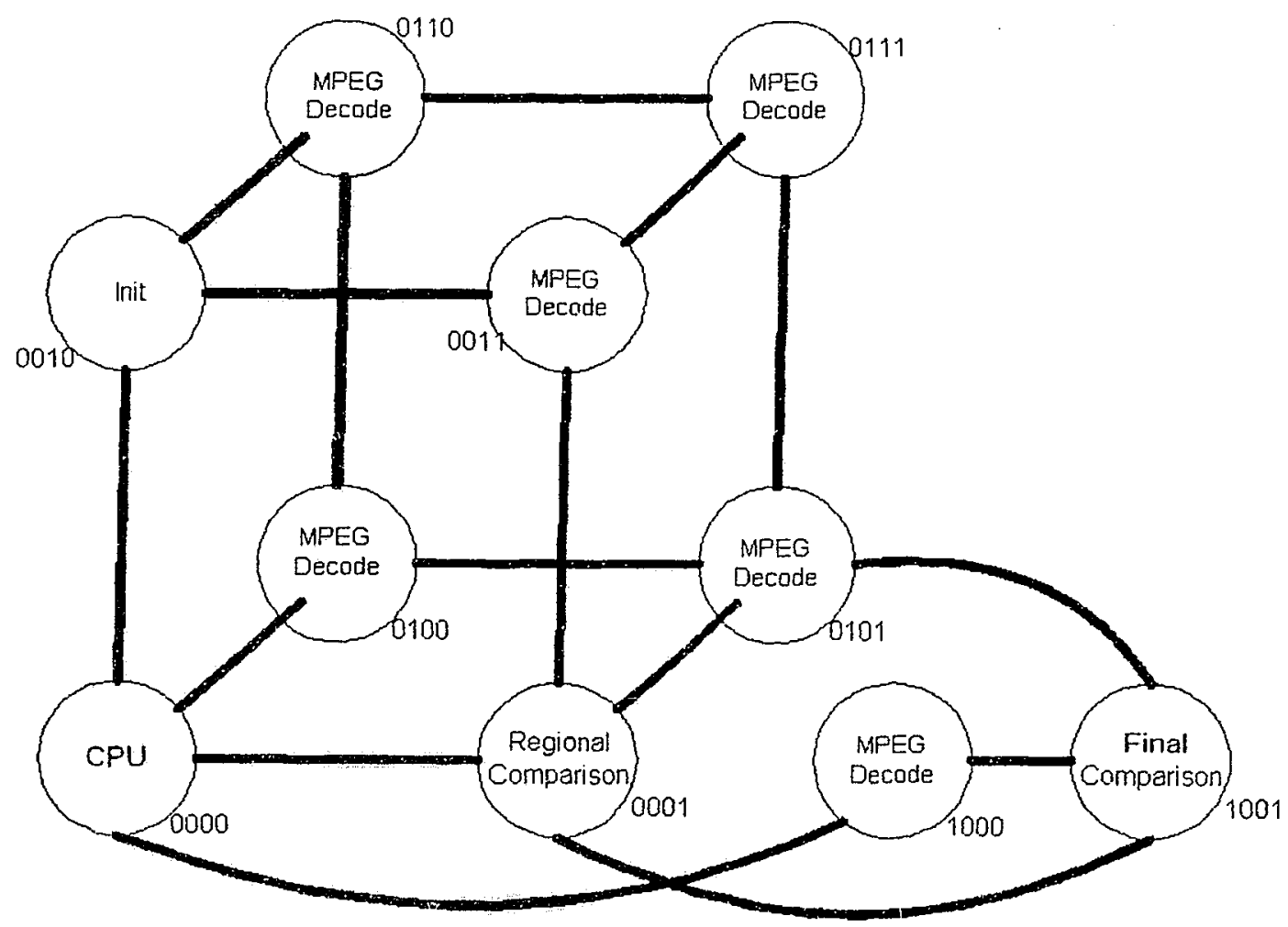

Figure 4.3: Parallel MPEG Decodis g: Architecture for $1^{\text {st }}$ Test Case 


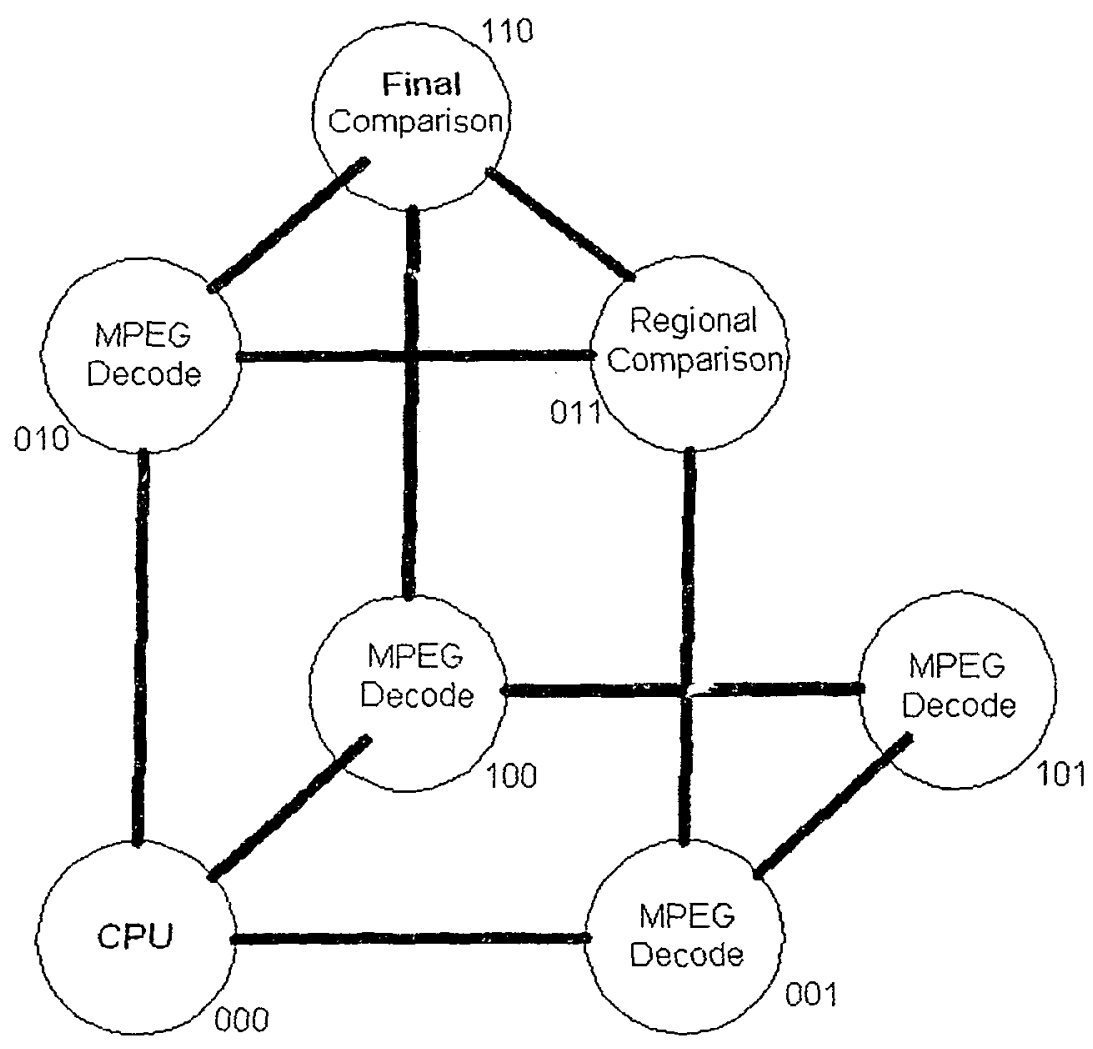

Figure 4.4: Parallel MPEG Decoding: Architecture for $2^{\text {nd }}$ Test Case 


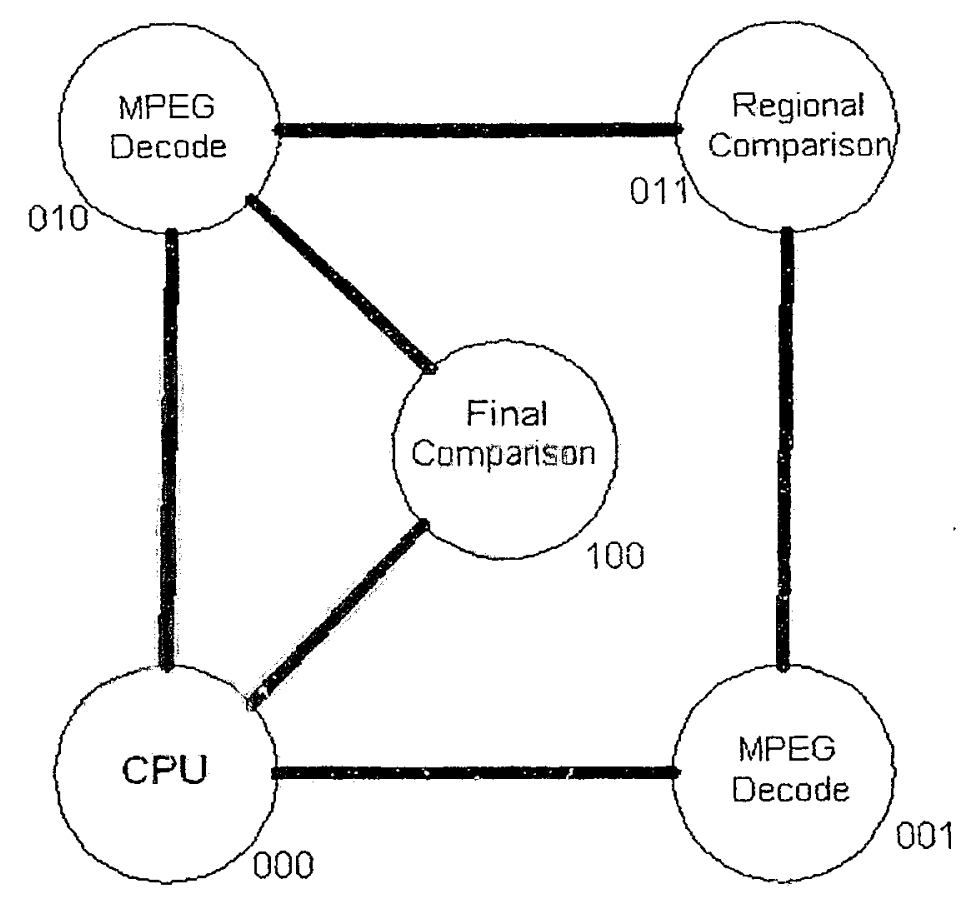

Figure 4.5: Parallel MPEG Decoding: Architecture for $5^{\text {th }}$ Test Case

\subsection{Parallel Block Matching}

An effective demonstration of the capabilities of the co-synthesis algorithm would have to involve an application that is computationally intensive. Additionally, for demonstrative purposes it is beneficial to implement a device that is easily understood. In the previous case study a device has been implemented that utilizes 16 MPEG decoders. MPEG decoding is the process by which a compressed video file is uncompressed for the purpose of viewing or accessing the raw image data. For the second case study, the block matching algorithm has been implemented. MPEG and other video compression formats are designed for fast transmission / easy storage of 
video data. In order for such a format to be useful, the decoding process must be relatively fast. In order to efficiently compress the video data for easy decoding, a computationally intensive encoding algorithm must be run. Block matching is the most time consuming component of MPEG encoding. Block matching analyzes the macroblocks from the input image and compares them with the surrounding area on the reference image. By analyzing all possible locations, an exhaustive block matching algorithm will generate motion vector data indicating the movement of a given macroblock from one image to the next.

A multiple PE embedded device responsible for performing the block matching algorithm has been implemented. Such a device could be used for any application involving the acquisition of video data. The device is fully specified in $\mathrm{C}$ language. A task graph representation is obtained by analysis of the specification and is provided in Figure 4.6. The functionality of the system is expressed as a graph of 22 tasks. The group based fault tolerance (GBFT) techniques previously outlined have been used and the resultant task grouping is provided in Figure 4.7. The GBFT grouping results in the addition of the assertion tasks shown in Figure 4.8. The final resultant task graph after GBFT has been performed and each group has merged to become a single task is provided in Figure 4.9. Further discussion of how the GBFT algorithm groups tasks together is provided in section 3.2.4. 


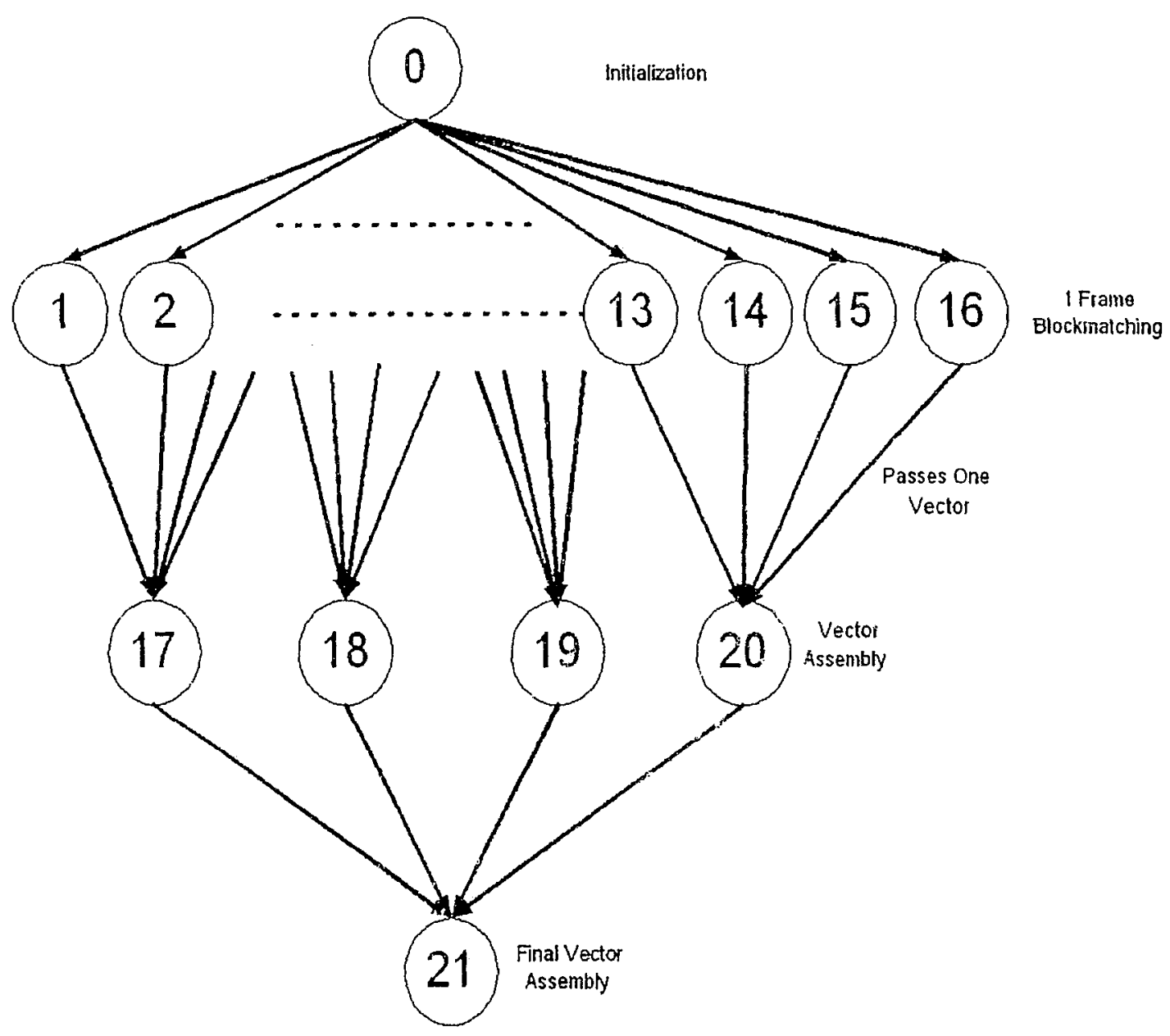

Figure 4.6: Block Matching: Functional Task Graph

Reproduced with permission of the copyright owner. Further reproduction prohibited without permission. 


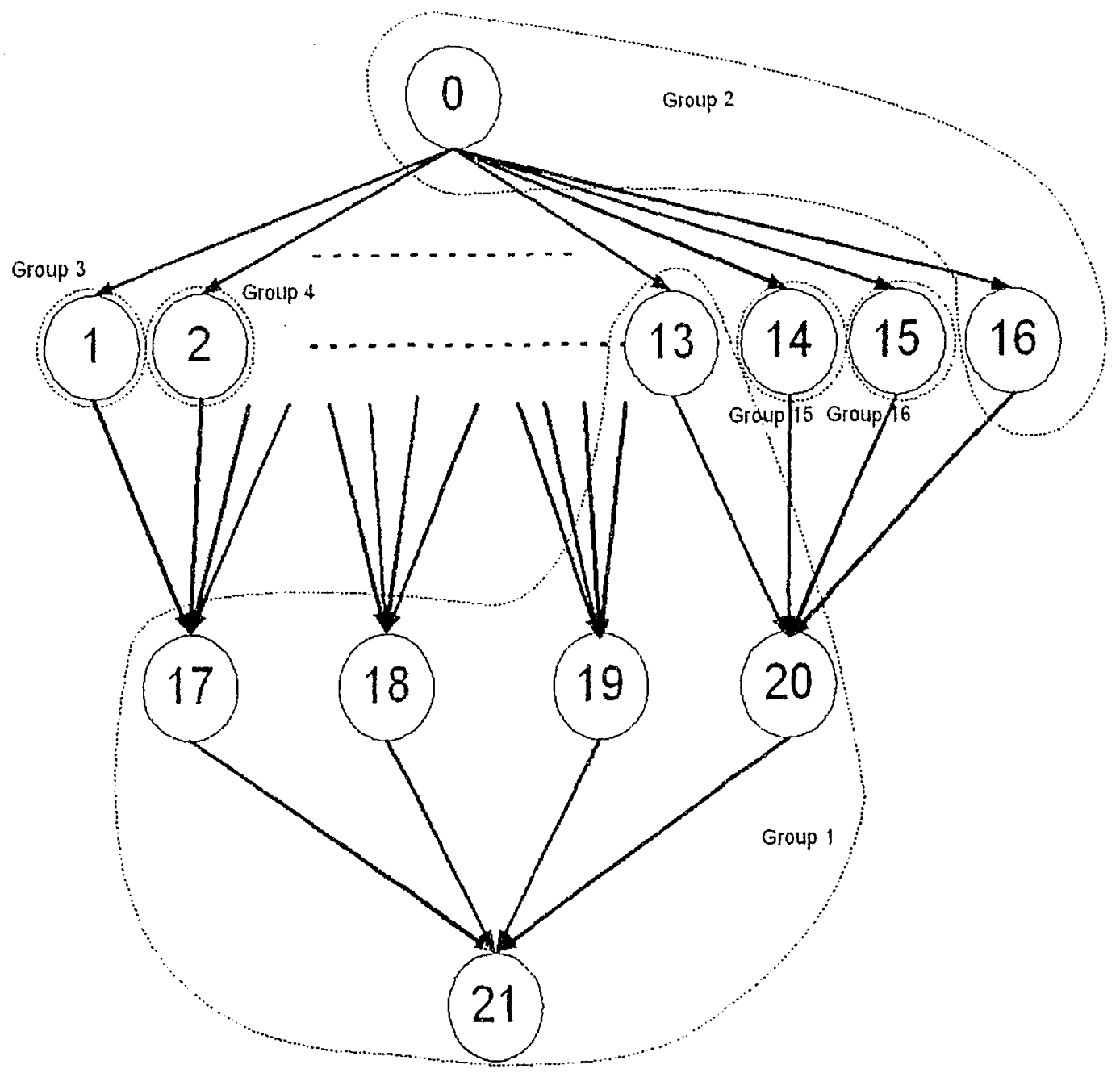

Figure 4.7: Block Matching Task Graph: GBFT Grouping 


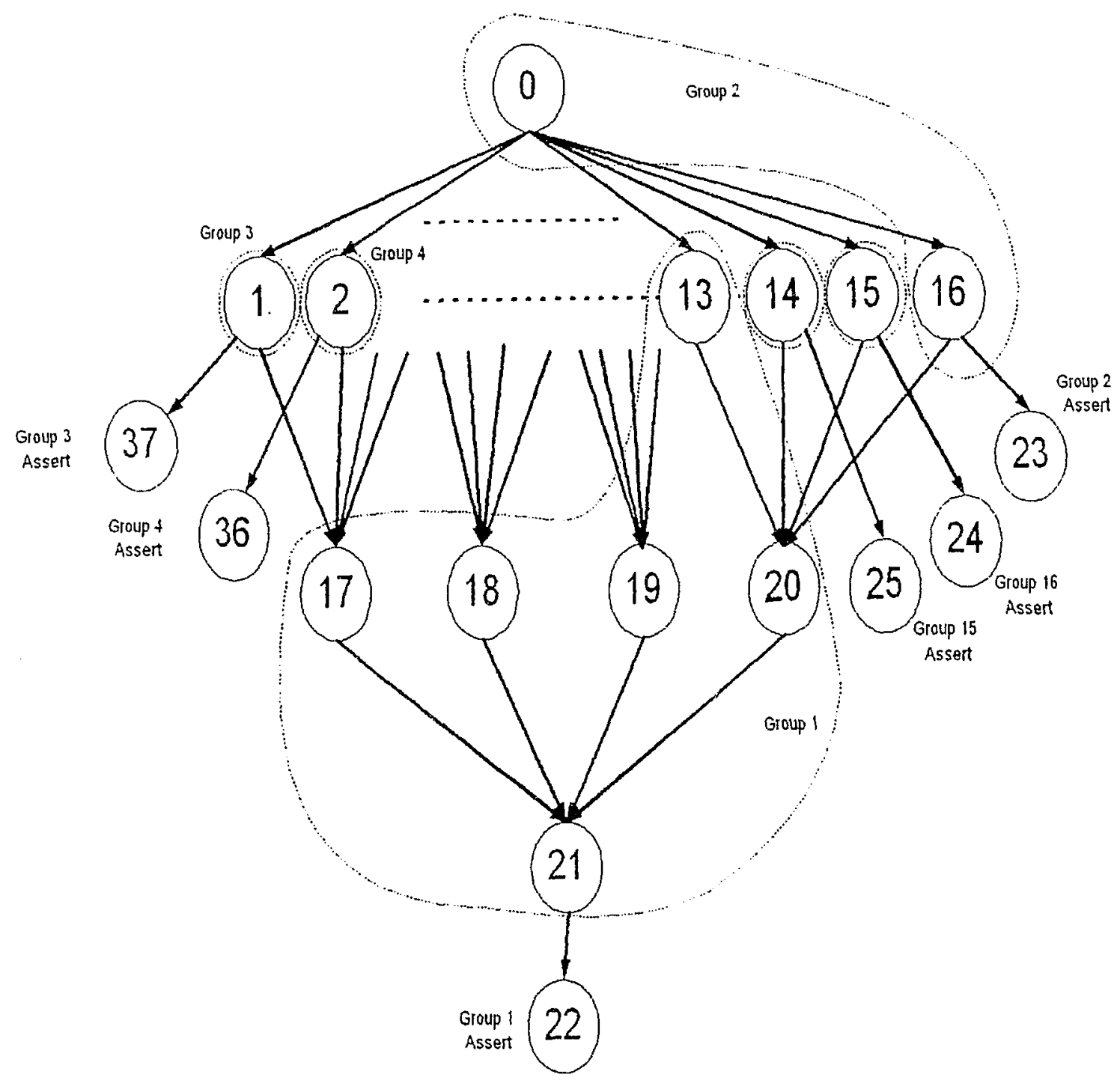

Figure 4.8: Block Matching Task Graph with Added Assertion Tasks 


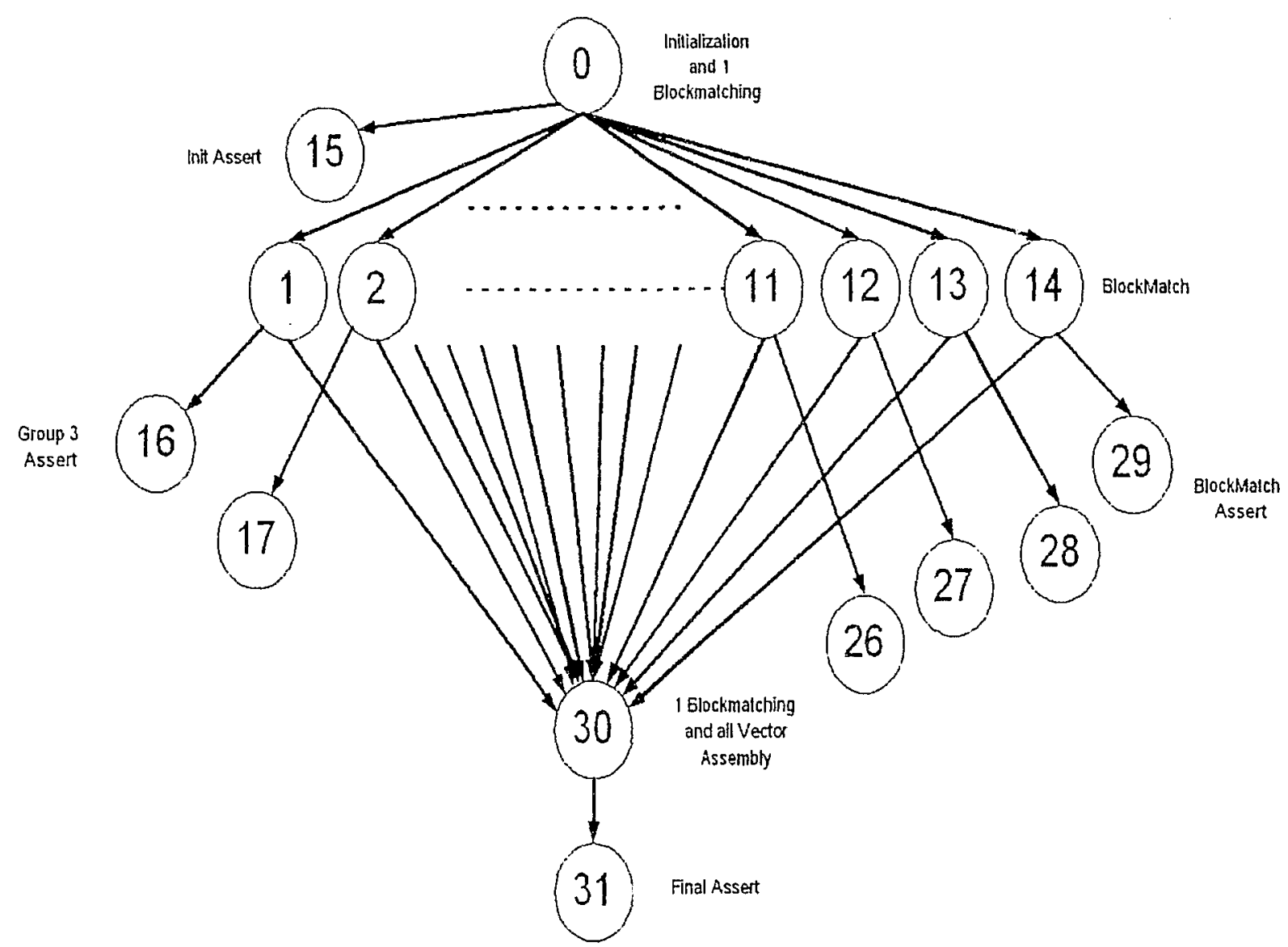

Figure 4.9: Block Matching: Final Resultant Task Graph

The most complex task in the device outlined is the block match task. In order to obtain pertinent hardware performance and area information for all tasks, the functionality needs to be implemented in hardware. This task has been implemented with the design provided in Figure 4.10. Yang et al. have presented an entire motion estimation architecture which utilizes a sub-circuit responsıble for error calculations that is similar to the implementation in Figure 4.10 [50]. Dutta and Wolf have presented a flexible motion estimation architecture [17] based on the research of Yang et al. In the design presented, the diff circuit calculates the absolute difference between the two input values. The absolute difference between the corresponding 
pixel values of the two input images is the error at the given point. The adder circuit adds the current errcr to the previously calculated errors to sum the overall error for a given vector. The comparison circuit compares the two input values and if the new value is lower than the old value, the circuit outputs the new value and latches the current I and $\mathrm{J}$ values into an internal register. These $\mathrm{I}$ and $\mathrm{J}$ values represent the current motion vector. The control unit tracks the current state of the circuit and signals all sub circuits, registers and outputs accordingly. A formal description of the functionality of the device is provided in the VHDL code in Appendix A.

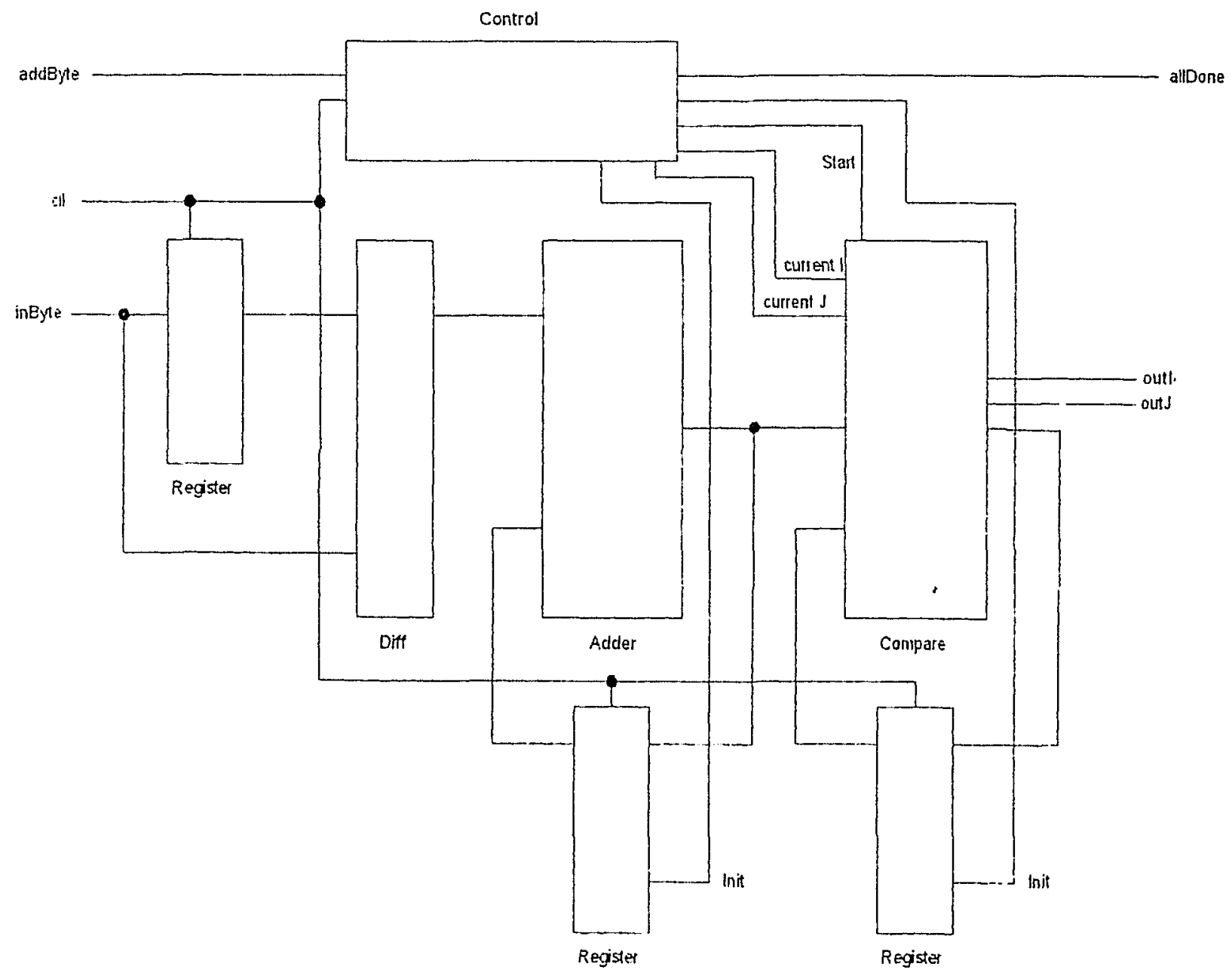

Figure 4.10: Block Match Circuit Design 
This block match circuit operates using a state machine which is administered in the control unit and is provided in Figure 4.11. State 0 is the initial state when the circuit is waiting for input. In state 1 the circuit has just received an even numbered count of inputs and as a result, the adder register is signaled to load the adder circuit generated value. In state 2 the circuit has just received an uneven number of inputs. If the circuit has received 512 input values, the circuit transitions to state 3 and signals the compare sub-circuit to begin execution, otherwise the circuit returns to state 1 . In state 3 the compare register is signaled to load the compare circuit generated error value. In state 4 the adder register is initialized and the incoming data is loaded into the input register. 


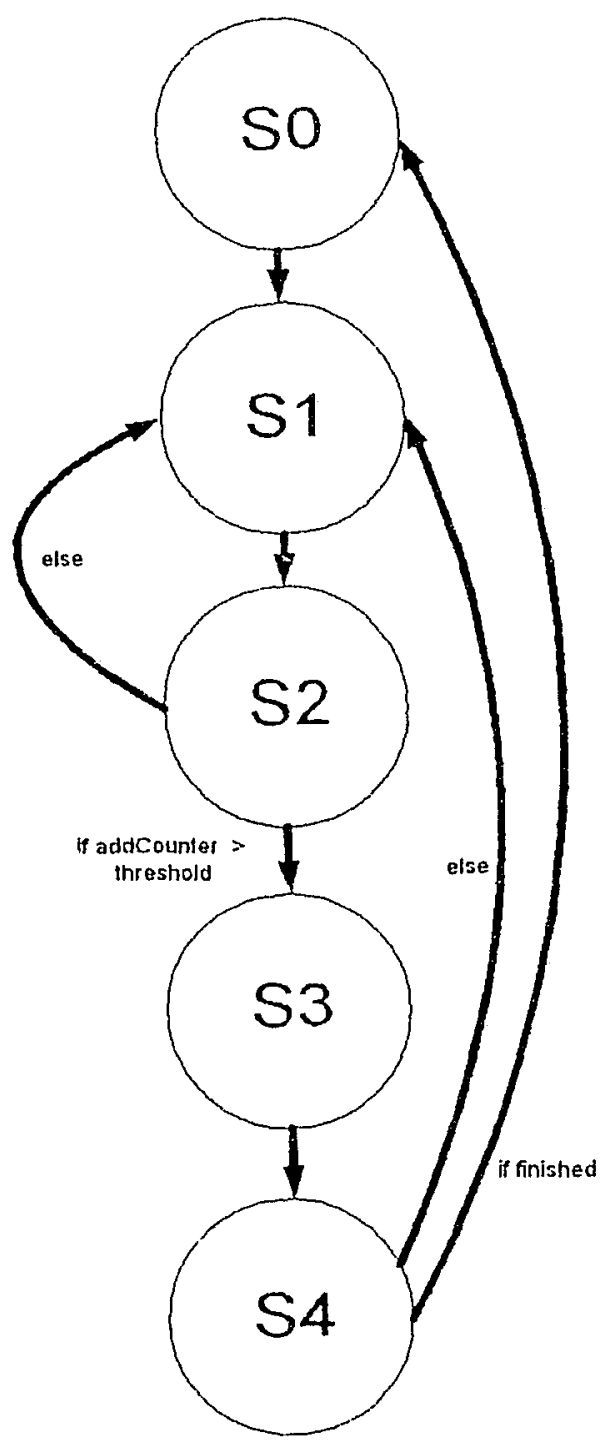

Figure 4.11: Block Match State Machine

The design space of the application in terms of device area and timing constraints has been tested in order to evaluate the performance of the approach. The test and experimental results are provided in Figure 4.12. A set of hardware and software implementation data was provided to the algorithm. In order to establish the software exccution times for each task, the $C$ language based specilication was profiled on an ARMTTDMI processor. The algorithm library can consist of many CPU types, 
however, for simplicity and practicality only the ARM7TDMI has been included for this case study. Each of the tasks were fully implemented in hardware and profiled for performance and hardware area requirements on a Xilinx VirtexE XCV2000E FPGA. Table 4.2 provides information regarding the quantity of each type of PE used for each of the design constraints studied.

The co-synthesis algorithm provides support for fault tolerant inter-PE communications by generating the target device into a hypercube architecture. By using the group-based fault tolerance (GBFT) method, fault tolerant embedded devices have been synthesized. The GBFT algorithm adds assertion and duplicate/compare tasks to the task graph. Upon detection of a failure, the assertion/compare task signals one of the spare PEs to commence execution of the failed :ask. The type and number of spare PEs are defined by the user.

In the previous case study the results were compared with those of an exhaustive technique. The exhaustive technique is very computationally intensive. On the 22 node task graph provided in the previous case study, the exhaustive technique required approximately 24 hours of execution time. The parallel block matching case study consists of 32 nodes (after GBFT) and as a result the exhaustive technique is far too computationally intensive to gather comparative results within a reasonable amount of time. As a result, in Figure 4.12 only the device constraints have been provided to our program and the resultant output provided by the algorithm have been included. 


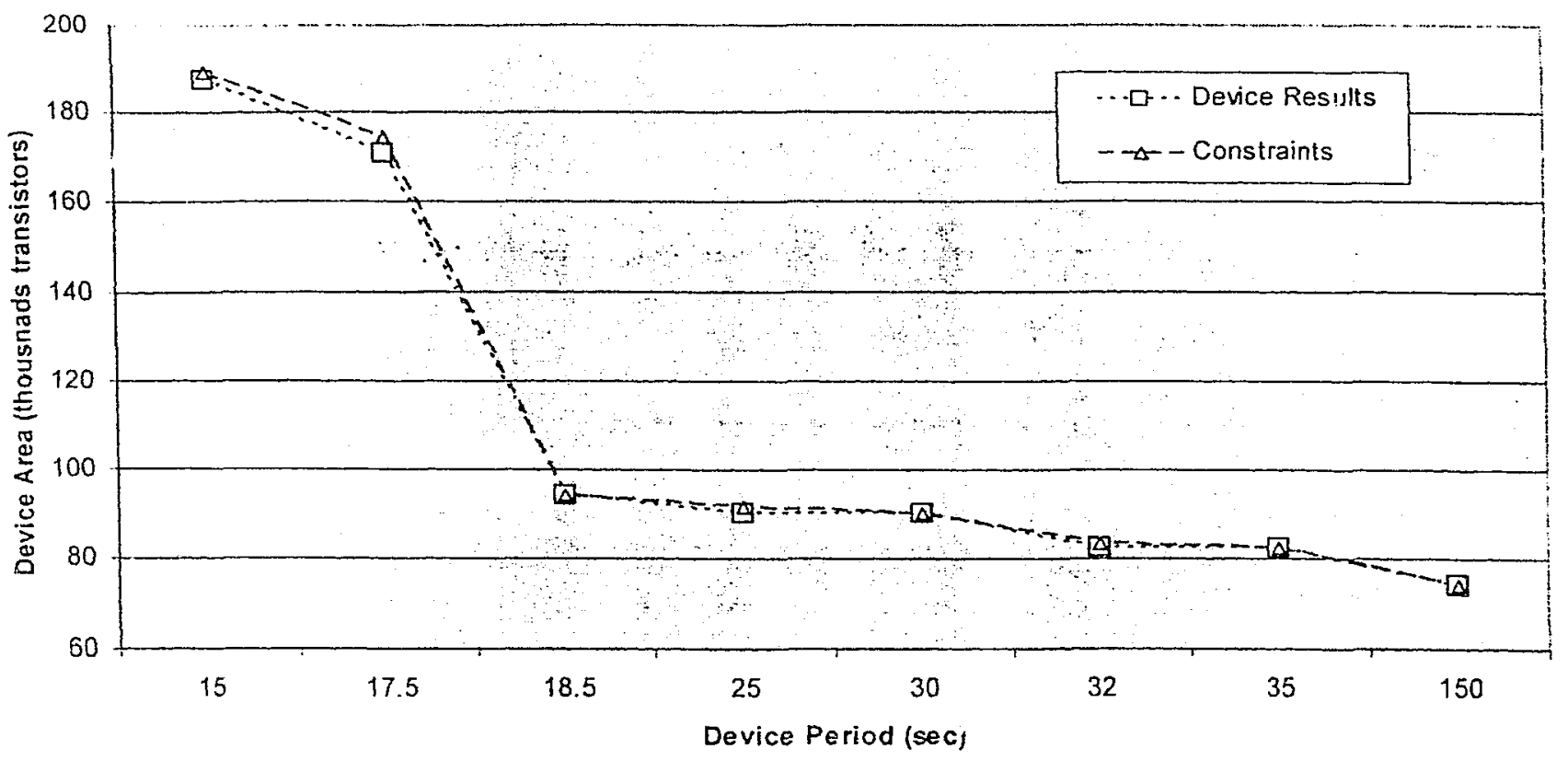

Figure 4.12: Design Space Exploration of Parallel Block Matching

The resulis provided in Figure 4.12 demonstrate the algorithm's ability to synthesize embedded devices with varying design constraints. A comparison of hardware area to performance trade-offs can be very useful to an embedded engineer in order to examine differences between design alternatives.

Reproduced with permission of the copyright owner. Further reproduction prohibited without permission. 
Table 4.2: Processing Element Utilization of Design Space for Block Matching

\begin{tabular}{|c|c|c|c|c|c|c|c|}
\hline $\begin{array}{c}\text { Area Constraint } \\
\text { (thousands of } \\
\text { transistors) }\end{array}$ & $\begin{array}{c}\text { Timing } \\
\text { Constraint } \\
\text { (sec) }\end{array}$ & $\begin{array}{c}\text { CPU } \\
\text { cores }\end{array}$ & $\begin{array}{c}\text { Block } \\
\text { Matching } \\
\text { Cores }\end{array}$ & $\begin{array}{c}\text { Block match } \\
\text { Assert Cores }\end{array}$ & $\begin{array}{c}\text { Final } \\
\text { Assert } \\
\text { Cores }\end{array}$ & $\begin{array}{c}\text { Total } \\
\text { PEs }\end{array}$ & $\begin{array}{c}\text { Exec. } \\
\text { Time } \\
\text { (msec) }\end{array}$ \\
\hline 189 & 15 & 2 & 3 & 1 & 1 & 7 & 342.4 \\
\hline 174 & 17.5 & 2 & 2 & 1 & 1 & 6 & 233.7 \\
\hline 94 & 18.5 & 1 & 2 & 1 & 1 & 5 & 144.0 \\
\hline 91 & 25 & 1 & 2 & 1 & 0 & 4 & 112.5 \\
\hline 90 & 30 & 1 & 2 & 1 & 0 & 4 & 153.7 \\
\hline 83 & 32 & 1 & 1 & 1 & 0 & 3 & 100.3 \\
\hline 82 & 35 & 1 & 1 & 1 & 0 & 3 & 112.5 \\
\hline 74 & 150 & 1 & 0 & 0 & 0 & 1 & 2.4 \\
\hline
\end{tabular}

Table 4.2 provides detailed information regarding the configuration of each solution that the co-synthesis algorithm produced. Given the design constraints provided in the first two columns, the algorithm generated a device that could meet specifications. The next four columns (3-6) provide the count of various ASIC circuits present in the final synthesized system. The total PEs column provides a count of the total number of PEs present in the given device. The count of initialization cores and final cores have not been included as there are none implemented in hardware for each case. The final column provides the length of time the algorithm took to execute in order to synthesize the device. 
In order to further demonstrate the efficacy of the approach a prototype device has been constructed based on the design generated by the hardware-software cosynthesis algorithm. A device based on the third set of design constraints provided in table 4.2 has been constructed. The algorithm was provided with an area constraint of 20000 gates in excess of the area of the initial CPU (74209 gates). The algorithm was also provided with a performance (period) constraint of 18.5 seconds. After cosynthesis, the algorithm generated a final device arrhitecture as seen in Figure 4.13. The design consists of a single CPU, two block matching PE circuits. one block match assert circuit and one final assert circuit. Additionally, in order to support fault tolerance one additional spare block matching circuit and two repeater circuits have been included in the design.

The algorithm predicted a final device area of 19683 gates in excess of the initial CPU area (74209). One block matching processing element and two repeater processing elements were added to support fault tolerance. A single block matching circuit has been synthesized and determined to utilize 3451 gates. The addition of three more processing elements also results in the addition of six new communication links. Each additional link results in the addition of two communication interfaces. Each additional communication interface has been synthesized and determined to utilize 354 gates. Finally, in order to suppori software to hardware communications on the ARM rapid prototyping platforn, additional interface circuitry is required. This circuitry was synthesized and determined to utilize 2866 gates. The algorithm also predicted a device period of 18.49 seconds. 
The sum of all of the hardware area predictions are as follows:

$\begin{array}{ll}19863 & \text { Gate count of initial hardware in excess of initial CPU } \\ 74209 & \text { Gate count of initial CPU } \\ 3451 & \text { Gate count of } 1 \text { additional block match circuit } \\ 4248 & \text { Gate count of } 12 \text { additional interface circuits }(12 * 354) \\ +\quad 2866 & \text { Gate count of ARM circuitry required for HW/SW comm. } \\ =\quad 104637 & \text { Total gate count }\end{array}$

The entire device represented by Figure 4.13 was implemented in over 2500 lines of VHDL and C code. A listing of the code is provided in Appendix A. Synthesis of the final device's non CPU hardware revealed an actual gate count of 28368 transistors. Adding the total gate count of the initial CPU (74209) yields a final device actual gate count of 102577 transistors.

The completed hardware-software device was profiled for performance. It was determined to have a period of 17.02 seconds. 


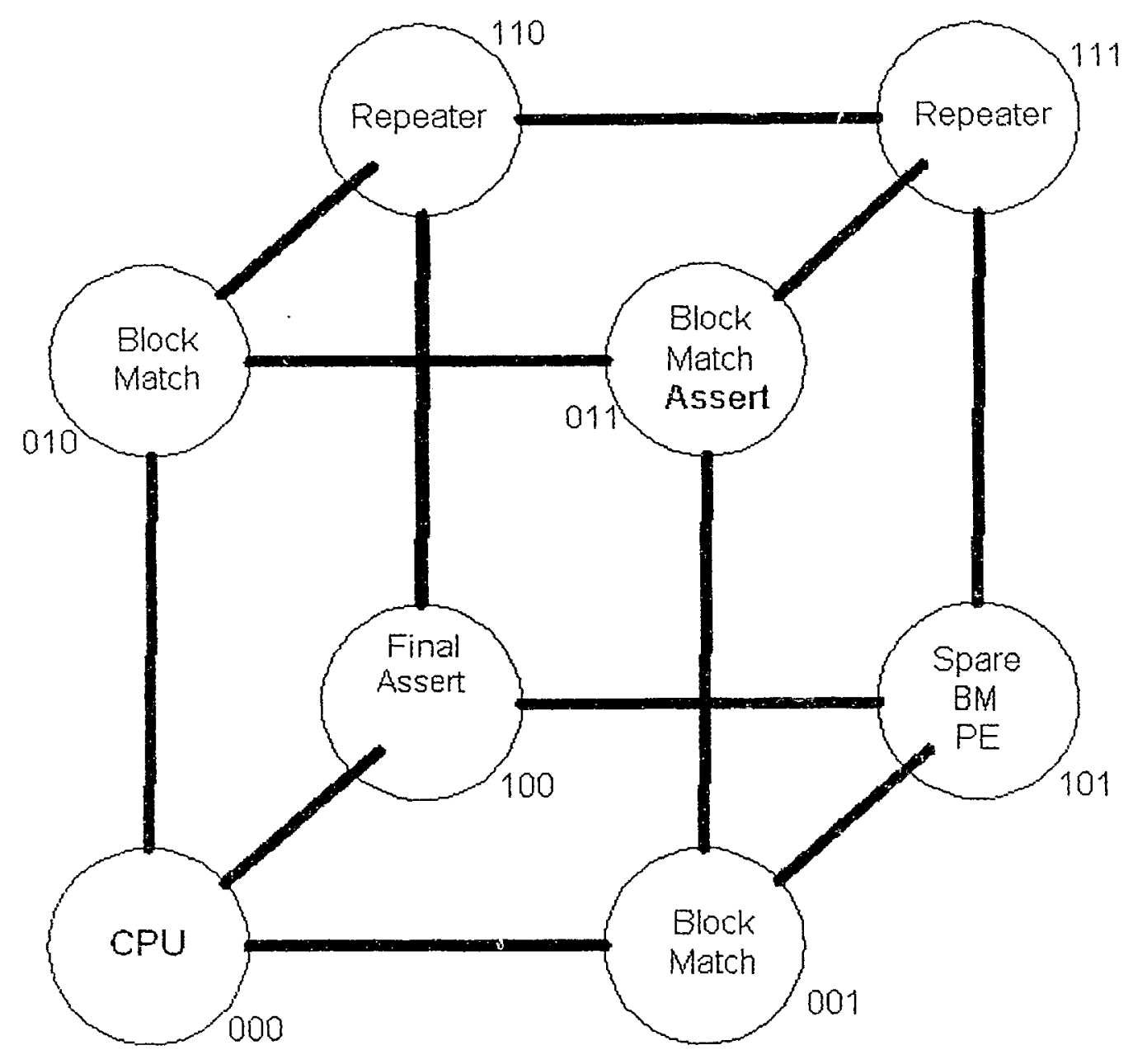

Figure 4.13: Final Prototype Device Architecture

The group based fault tolerance method developed allows the user to manually select the quantity and type of spare processing elements. In order to support fault tolerance but to also limit the fault tolerance overhead one spare block matching processing element has been included. Additionally, in order to simplify the hypercube topology and for ease of link fault tolerance, two repeater processing elements have been included. The repeater processing elements consist solely of communication 
interface circuitry, and are only responsible for repeating incoming data on the appropriate outgoing link.

In order to test the device's functionality, an input image and a reference image have been provided as input. The device then calculated the motion vectors for each of the 16 macroblocks in the images. The reference image is provided in Figure 4.14. The input image is provided in Figure 4.15. The macroblock numbering convention used is provided in Figure 4.16. The generated vector data is provided in Table 4.3.

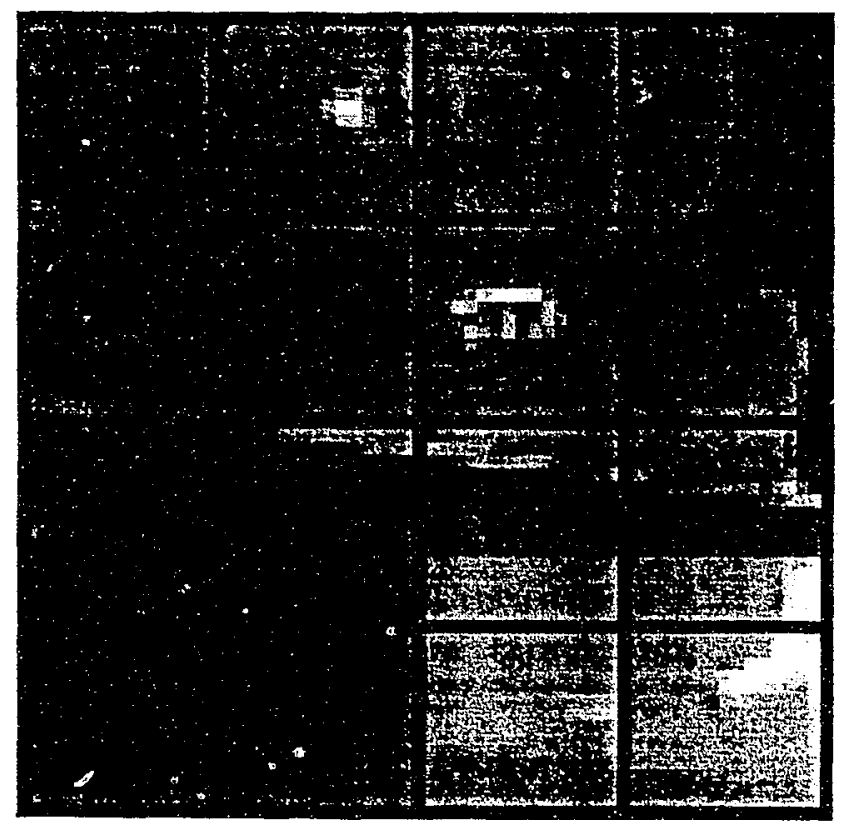

Figure 4.14: Reference Image 


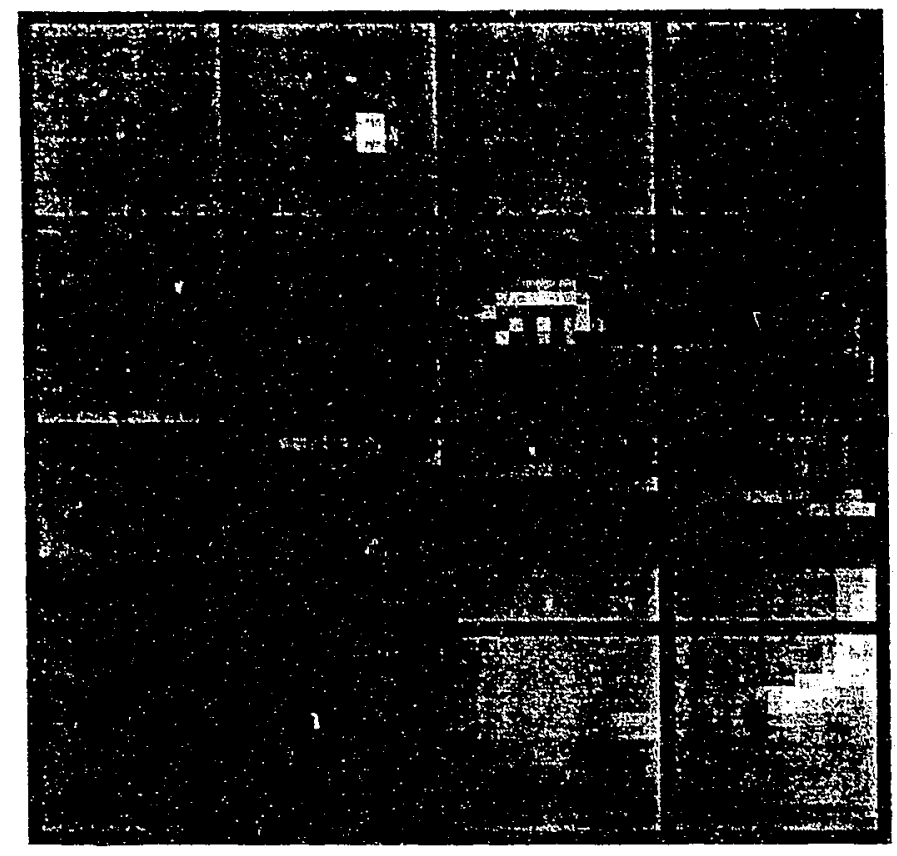

Figure 4.15: Input Image

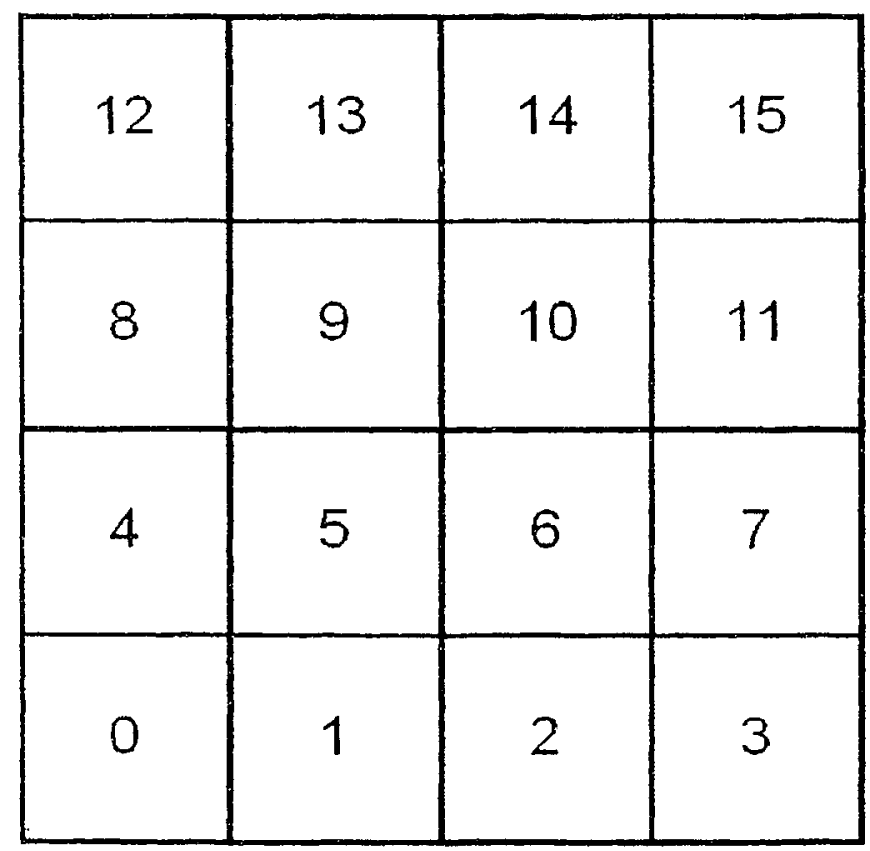

Figure 4.16: Macroblock Identification 
Table 4.3: Generated Motion Vectors

\begin{tabular}{|c|c|c|}
\hline Macrobiock & Row Shift & Column Shife \\
\hline 0 & 0 & 0 \\
\hline 1 & 0 & 0 \\
\hline 2 & 0 & 0 \\
\hline 3 & 0 & 0 \\
\hline 4 & 0 & 0 \\
\hline 5 & -1 & -1 \\
\hline 6 & 0 & 0 \\
\hline 7 & 0 & 0 \\
\hline 8 & 0 & 0 \\
\hline 9 & 0 & 0 \\
\hline 10 & 0 & 0 \\
\hline 11 & 0 & 0 \\
\hline 12 & 0 & 0 \\
\hline 13 & -2 & 0 \\
\hline 14 & 0 & 0 \\
\hline 15 & 0 & 0 \\
\hline
\end{tabular}

In order to confirm that the device correctly supports fault tolerance error states were simulated. Random error conditions in various block matching subcomponents were simulated by using a random number generator. After an error state has been detected, all further communication to the block matching PE where the error was detected is rerouted to one of the spare block matching PEs. Additionally. the set of tasks executed on the PE where the error was detected must be re-executed on the newly activated spare PE. This results in a degradation of overall system performance for the period in which the error was detected. The average measured period of the device when an error was detected is 20.94 seconds. The output results from the cases where an error was detected is identical to those generated by the device when no error was detected. 


\subsection{Discussion of Experimental Results}

A design methodology for medium to large-scale fault-tolerant embedded systems has been presented. The objective has been to introduce a tool to aid the embedded systems developer to create hypercube devices meeting performance, cost and reliability constraints within a reasonable amount of time. To achieve this goal efficiently and effectively, a series of equations were developed to govern pipelined scheduling, task reallocation, addition of processing elements, configuration of processing elements within a hypercube topology and synthesis of inter-PE communication links. One of the predominant motivations is to minimize undesirable multi-hop communications that can occur in hypercube systems. Additionally, the innate link-fault tolerant nature of hypercube architectures is preserved. Support for fault tolerance has been developed through the use of the group based fault tolerance technique.

In order to demonstrate the efficacy of the new group based fault tolerance technique, the algorithm was compared to both cluster and task based fault tolerance. To the best of our knowledge cluster and task based fault tolerance are the only other published methods governing the addition of error detection functionality at the task graph level. The experimental results showed that on average the group based fault tolerance method yields a $9.8 \%$ improvement in fault tolerant overhead over the cluster based fault tolerance method and a $61 \%$ improvement over the task based fault 
tolerance method. These results demonstrate the efficacy of the group based fault tolerance method.

In order to evaluate the efficacy of the co-synthesis approach the results of an optimal algorithm have been provided for the test cases of the first case study. The exhaustive technique is a simple approach that attempts all possible combinations and is extremely computationally intensive. This exhaustive technique was implemented for the sole purpose of evaluating the results of the algorithm with the optimal solution. On average the approach yielded devices that under-performed the optimal case by $3.75 \%$. Additionally, the algorithm resulted in designs that use $0.62 \%$ more hardware area than that of the optimal approach. These results show only small deviations between the algorithrn's solutions and the ideal ones, indicating that the proposed method yields high quality solutions. To the best of our knowledge, this approach is the first co-synthesis algorithm developed targeting embedded hypercube systems. Comparing the results of this approach with, for instance, a co-synthesis algorithm developed to target bus systems would be inconclusive due to the differences in the target architectures. Consequently, the comparison has been limited to that of the optimal method.

Another common approach for comparing various co-synthesis or partitioning techniques is to evaluate their respective execution times. Some timing analysis of the algorithm has been performed with relation to the case studics presented in this paper (see Tables $4.1 \& 4.2$ ). It can be observed that as the hardware constraint rises, 
so does the execution time. As more hardware is added to the system, more itcrations of the algorithm must be executed. The average execution time for the algorithm (across both case studies) is $84.92 \mathrm{~ms}$. Synthesizing the 22-task embedded device (first case study) for the given set of input requirements would take approximately 24 hours to complete using the optimal approach. The execution time of such an approach is impractical for the comparable task graphs of the case study devices. Additionally, it is expected that the execution time would increase exponentially if the number of tasks in the device specification increases. This was verified when attempting to use the optimal method on the 32-node task graph from the second case study. The optimal execution time was observed to be prohibitive and as a result no optimal cases were completed. In the cases where the optimal technique could be used, it was found to produce marginally better designs but its execution time was too large and impractical. Both algorithms were executed on a Pentium IV $2.8 \mathrm{GHz}$ system with $512 \mathrm{MB}$ of memory. The execution timing results indicate that the approach is efficient and its execution time is not a serious impediment to its performance. The first case study presented in this paper is fully implemented and executed in simulation.

In order to further confirm the effectiveness of the devices that the algorithm produces. a prototype of the second case study device has been built using a rapid prototyping platform based on the ARM CPU and Xilinx VirtexE FPGA. 
The results show a final device gate count of 102577 transistors. As discussed in the previous section, the algorithm predicted a total device gate count of 104637 transistors. These predicted results yield a $2.0 \%$ error. This error has been attributed to the estimates on the cost of the communication link interfaces. The interface gate count was estimated by synthesizing a standard communication interface. The largest source of the discrepancy comes from the communication link interface circuitry that accompanies the CPU processing element. Due to the complex nature of the CPU, this interface circuitry deviates considerably from that of the other processing elements. Additionally, the VHDL compiler attempts to optimize the device area which may reduce the overall device gate count. The existence of these discrepancies was not realized until the final device was completed, as a result the modeling of these costs into the actual system was not possible.

The results show a final device performance period of 17.02 seconds. The algorithm predicted a final device period of 18.49 seconds. This is an $8.6 \%$ error. This error has been attributed to the observed inconsistency in the time measurement functions available for use with the ARM CPU. During profiling, the same functionality was repeatedly measured for timing. The execution time of the same software functional block was observed to typically vary by approximately $10 \%$ and in some cases as much as $40 \%$. Due to this discrepancy, estimated execution times based on the maximum measured values were selected. This is believed to be the main source of error contributing to the overall device period estimate being $8.6 \%$ higher than the final measured value. 
It should be noted that with a period of 17.02 seconds, the prototype device is slow. This is due to us using an ARM7TDMI processor. The ARM7 is widely used in small scale embedded systems and at 74,209 transistors, it is an extremely small processor. By comparison, an old desktop CPU such as the Intel Pentium II, consists of 7.5 million transistors which is approximately 100 times larger. Additionally the communication bus on the rapid prototyping platform is also slow. 


\section{CHAPTER 5}

\section{CONCLUSIONS AND FUTURE WORK}

The hardware-software co-synthesis of an embedded system architecture involves the partitioning of a system specification or functional description into hardware and software modules so as to meet a series of non-functional requirements such as cost and performance. Various distributed embedded device architectures have been presented previously, including bus, hierarchical and hypercube. Hypercube architectures are particularly suitable for use in fault tolerant and high performance devices. To the best of our knowledge, the first co-synthesis algorithm has been presented that will automatically generate fault tolerant hypercube architecture based embedded devices. The newly developed group based fault tolerance, a technique for adding fault tolerance to an embedded device at the task graph level has also been presented. The co-synthesis algorithm consists of six main steps: specification defining device functional and non-functional requirements, profiling - evaluating the functional specification for performance and area utilization, group based fault tolerance - adding fault tolerance to the device at the task graph level, scheduling evaluating the performance of the current device architecture, addition of processing elements - improving device performance by adding more hardware, and the synthesis of communication links - arranging all processing elements within a hypercube topology and synthesizing all communication links. The algorithm attempts to minimize the occurrence of multiple hops during inter-task 
communications. This helps to reduce the overall system communication overhead and thus increases device performance.

A security/navigation device has been implemented that is responsible for decoding $16 \mathrm{MPEG}$ video streams in parallel. The co-synthesis algorithm's final architecture results were compared with that of the optimal case. This comparison revealed only minor deviations between the co-synthesis algorithm's generated devices and that of the optimal technique. These minor deviations illustrate how the co-synthesis algorithm is capable of generating high quality solutions.

A second device has been implemented to illustrate the algorithm's ability to generate fault tolerant devices. This device is responsible for performing the block matching algorithm which is a computationally intensive and an essential component of MPEG encoding. Additionally, in order to further demonstrate the efficacy of the approach, a prototype device based on the algorithm's results has been implemented. The final prototype device's area and timing values were compared with the algorithm's predicted values and revealed only modest deviations from the anticipated results. Error conditions were also simulated on the prototype device to confirm correct fault tolerant device functionality. This demonstrates the co-synthesis algorithm's ability to generate fault tolerant devices and to accurately predict device area and performance costs. 
During the synthesis and development of the block matching case study, available equipment was limited to a small ARM7TDMI CPU connected to a Xilinx FPGA via a slow communication medium. This resulted in a slow performing but inexpensive device. Future work will entail the development of an additional prototype based on a faster and larger CPU. Additionally, the system will have a faster FPGA for the application specific circuitry and higher speed hardware-software communications. This will further demonstrate our co-synthesis algorithm's capabilities by synthesizing and building a prototype device with more practical performance and area constraints.

The co-synthesis algorithm presented, targets fault tolerant embedded devices and takes as input functional and non-functional requirements. The non-functional requirements include performance and area. In order to further enhance the algorithm's ability to synthesize fault tolerant devices, future work will entail adapting the algorithm to include reliability as a non-functional requirement. CPUs, ASICs and communication links all have an inherent reliability factor and the overall device reliability will be a function of the selection of differing processing elements, links and how they are interconnected. 


\section{REFERENCES}

[1] P. Athanas, H. F. Silverman, "Processor reconfiguration through instruction-set metamorphosis", IEEE Computer, vol. 26, no. 3, pp. 11-18, March 1993.

[2] S. Bakshi and D. Gajski, "Partitioning and Pipelining for PerformanceConstrained Hardware/Software Systems," IEEE Transactions on Very Large Scale Integration Systems, vol. 7, no. 4, Dec. 1999.

[3] E. Barros, W. Rosenstiel, and X. Xiong, "A method for partitioning UNITY language to hardware and software," Proceedings European Design Automation Conference, Sept 1994, pp. 220-225, Grenoble, France.

[4] C. Bolchini, L. Pomante, F. Salice, D. Sciuto, "Online Fault Detection in a Hardware/Software Co-Design Environment: System Partitioning", Proceedings IEEE International Symposium on System Synthesis, Oct. 2001, pp. 51-56, Montreal, Canada.

[5] Jui-Ming Chang, Massoud Pedram, "Codex-dp: Co-Design of Communicating Systems Using Dynamic Programming", IEEE Transactions on Computer-Aided Design of Integrated Circuits and Systems, vol. 19, no. 7, July 2000.

[6] K. S. Chatha and R. Vemuri, "Hardware-Software Partitioning and Pipelined Scheduling of Transformative Applications," IEEE Transactions on Very Large Scale Integration Systems, vol. 10, no. 3, June 2002.

[7] K. S. Chatha and R. Vemuri, "RECOD: a retiming heuristic to optimize resource and memory utilization in HW/SW codesigns," Proceedings International Workshop on Hardware/Software Codesign, March 1998, pp. 139-143, Seattle, USA.

[8] J. Choquette, M. Gupta, D. McCarthy, J. Veenstra, "High performance RISC microprocessors", IEEE Micro, pp. 48-55, Aug 1999.

[9] L.A. Cortes, P. Eles, Z. Peng, "Formal coverification of embedded systems using model checking", Proceedings Euromicro Conference, Sept. 2000, vol. 1, pp. 106-

113, Maastricht, Netherlands.

[10] L.A. Cortes, P. Eles, Z. Peng, "Verification of embedded systems using a Petri net based representation", Proceedings International Symposium on System Synthesis, Sept. 2000, pp. 149-155, Madrid, Spain.

[11] J. G. D'Ambrosio and X. Hu, "Configuration-level hardware/software partitioning for real-time systems," Proceedings International Workshop HardwareSoftware Co-Design, Sept. 1994, pp. 34-41, Grenoble, France.

[12] S. K. Das, M. C. Pinotti anr" ". Sarkar, "Optimal and load balanced mapping of parallel priority queues in hypercubes," IEEE Transactions on Parallel and Distributed Systems, vol. 7, issue 6, pp. 555-564, June 1996.

[13] B. P. Dave and N. K. Jha, "COFTA: Hardware-software co-synthesis of heterogeneous distributed embedded system architectures for low overhead fault tolerance," IEEE Transactions on Computers, vol. 48, no. 4, April 1999.

[14] B. P. Dave and N. K. Jha. "COHRA: Hardware-Software Cosynthesis of Hierarchical Heterogeneous Distributed Embedded Systems," IEEE Transactions on Computer Aided Design of Integrated Circuits and Systems, vol. 17, no. 10, Oct. 1998. 
[15] B. P. Dave, G. Lakshminarayana and N. K. Jha, "COSYN: Hardware-software co-synthesis of heterogeneous distributed embedded systems," IEEE Transactions on VLSI Systems, vol. 7, no. 1, March 1999.

[16] R. Dick and N. K. Jha, "MOGAC: A Multiobjective Genetic Algorithm for Hardware-Software Cosynthesis of Distributed Embedded Systems," IEEE Transactions on Computer-Aided Design of Integrated Circuits and Systems, vol. 17. no. 10, pp. 920-935, Oct. 1998.

[17] S. Dutta and W. Wolf "A Flexible Parallel Architecture Adapted to BlockMatching Motion-Estimation Algorithms", IEEE Transactions on Circuits and Systems for Video Technology, Vol. 6, No. 1, pp. 74-86, Feb. 1996.

[18] R. Ernst, J. Henkel, and T. Benner, "Hardware-software cosynthesis for microcontrollers," IEEE Design \& Test, vol. 10, pp. 64-75, Dec. 1993.

[19] F. C. Filho, P. Maciel, E. Barros, "A Petri Net Based Approach For Hardware/Software Partitioning", Symposium on Integrated Circuits and Systems Design, Sept. 2001, pp. 72-77, Pirenopolis, Brazil.

[20] M.R. Garey and D. S. Johnson, Computers and Interactability: A Guide to the Theory of NP-Completeness. San Francisco, CA: Freeman, 1979.

[21] C. Ghezzi, M. Jazayeri, D. Mandrioli, Fundamentals of Software Engineering, Prentice Hall, Upper Saddle River, NJ, 1991.

[22] R. Gupta and G. DeMicheli, "Hardware/software cosynthesis for digital systems," IEEE Design \& Test of Computers, pp. 29-41, Sept. 1993.

[23] R. Gupta and G. DeMicheli, "System-level synthesis using re-programmable components", Proceedings European Conference on Design Automation, Mar. 1992, pp. 2-7, Brussels, Belgium.

[24] J. Henkel, R. Emst, "An Approach to Automated Hardware/Software Partitioning Using a Flexible Granularity that is Driven by High-Level Estimation Techniques", IEEE Transactions on Very Large Scale Integration Systems, vol. 9, no. 2, April 2001. [25] J. Hou and W. Wolf, "Process partitioning for distributed embedded systems," Proceedings International Workshop on Hardware/Software Codesign, Sept. 1996. pp. 70-76, Pittsburgh, USA.

[26] P.-A. Hsiung, "Hardware-software timing coverification of concurrent embedded real-time systems", IEE Proceedings Computers and Digital Techniques, pp. 83-92, March 2000.

[27] B. A. Izadi, F. Ozguner, "Real-time fault-tolerant hypercube multicomputer," IEE Proceedings Computers and Digital Techniques, vol. 149, no. 5, pp. 197-202, Sep. 2002.

[28] A. Kalavade and E. A. Lee, "A hardware-software codesign methodology for DSP applications," IEEE Design \& Test, vol. 10, pp. 16-28, Sept. 1993.

[29] G. N. Khan, G. S. Hura, G. Wei, "Distributed Recovery Block Based Faulttolerant Routing in Hypercube Networks," Proceedings IEEE Canadian Conference on Electrical and Computer Engineering, May 2002, pp. 603-608, Winnipeg, Canada.

[30] D. Kirovski and M. Potkonjak, "System-level synthesis of low-power real-time systems," Proceedings Design Automation Conference, June 1997, pp. 697-702. Anaheim, USA. 
[31] P. V. Knudsen, J. Madsen, "PACE: A dynamic programming algorithm for hardware/software partitioning", Proceedings $4^{\text {th }}$ International Workshop on Hardware/Sofiware Codesign, pp. 85-92, 1996, Pittsburgh, USA.

[32] K. Kuchcinski, "Constraints-driven scheduling and resource assignment," $A C M$ Transactions on Design Automation of Electronic Systems, vol. 8, no. 3, pp. 355-383, July 2003.

[33] Yu-Kwong Kwok and I. Ahmad, "Dynamic critical-path scheduling: An effective technique for allocating task graphs to multiprocessors," IEEE Transactions on Parallel Distributed Systems, vol. 7, pp. 506-521, May 1996.

[34] L. E. LaForge, "Self-Healing Avionics for Starships," Proceedings IEEE Aerospace Conference, March 2000, vol. 5, pp. 499-519, Big Sky, USA.

[35] L. E. LaForge, K. F. Korver and M. S. Fadali, "What Designers of Bus and Network Architectures Should Know about Hypercubes," IEEE Transacions on Computers, vol. 52, no. 4, April 2003.

[36] Y. Li and W. Wolf, "Hardware/Software Co-Synthesis with Memory Hierarchies," IEEE Transactions on Computer-Aided Design of Integrated Circuits and Systems, vol. 18, no. 10 pp. 1405-1417, Oct. 1999.

[37] Huiqun Liu, D. F. Wong, "Integrated Partitioning and Scheduling for Hardware/Software Co-Design", Proceedings International Conference on VLSI in Computers and Processors, Oct. 1998, pp. 609-614, Austin, USA.

[38] J. Madsen, J. Grode, P.V. Knudsen, M.E. Peterson, A. Haxthausen, "LYCOS: the lyngby co-synthesis system", Design Automation for Embedded Systems, vol. 2, no. 2, pp. 195-236, 1997.

[39] D.D. Mitchell, D.C. Ku, F. Mailhot, and T. Truong, "The Olympus Synthesis System for digital design", IEEE Design and Test Magazine, pp.37-53, Oct. 1990.

[40] A. Osterling, T. Benner, R. Ernst, D. Herrmann, T. Scholz, and W. Ye, "The COSYMA system", Hardware/Software Co-Design: Principles and Practice, pp. 263-281. Kluwer Academic Publishers, Amsterdam, 1997.

[41] D. A. Patterson, J. L. Hennessy, Computer Architecture A Quantitative Approach $2^{\text {nd }}$ Edition, Morgan Kaufman Publishers Inc., San Francisco, CA, 1996.

[42] S. Prakash and A. Parker. "SOS: Synthesis of application-specific heterogeneous multiprocessor systems." Journal of Parallel and Distributed Computing, vol. 16, pp. 338-351, Dec. 1992.

[43] K. Rajan, L. M. Patnaik and J. Ramakrishna, "High-speed parallel implementation of a modified PBR algorithm on DSP-based EH topology," IEEE Transactions on Nuclear Science, vo ! i, issue 4, pp. 1658-1672, Aug. 1997.

[44] E. Salminen, T. Hamalainen, T. Kangas, K. Kuusilinna, J. Saarinen, "Interfacing multiple processors in a system-on-chip video encoder", International Symposium on Circuits and Systems, May 2001, vol. 4, pp. 478-481, Sydney, Australia.

[45] Aviral Shrivastava. Mohit Kumar, Sanjiv Kapoor, Shashi Kumar, M.

Balakrishnan, "Optimal Hardware/Software Partitioning for Concurrent Specification using Dynamic Programming". International Conference on VLSI Design, Jan. 2000, pp. 110-113, Calcutta. India. 
[46] S. Srinivasan and N. K. Jha, "Hardware-Software Co-Synthesis of Fault-Tolerant Real-Time Distributed Embedded Systems," Proceedings European Design Automation Conference, Sept. 1995, pp. 334-339, Brighton, UK.

[47] W. Wolf, "An architectural co-synthesis algorithm for distributed, embedded computing systems," IEEE Transactions on VLSI Systems, vol. 5, pp. 218-229, June 1997.

[48] W. Wolf, Computers as Components, Morgan Kaufman Publishers Iric., San Diego, CA, 2001.

[49] S. Yajnik, S. Srinivasan, N. K. Jha, "TBFT: A Task Based Fault Tolerance Scheme for Distributed Systems," Proceedings International Conference on Parallel and Distributed Computing Systems, Oct. 1994, pp. 483-489, Las Vegas, USA. [50] Kun-Min Yang, Ming-Ting Sun and Lancelot Wu, "A Family of VLSI Designs for the Motion Compensation Block-Matching Algorithm", IEEE Transactions on Circuits and Systems, vol.36, no.10, pp. 1317-1325, Oct. 1989.

[51] Yao-Ming Yeh and Yiu-Cheng Shyu, "Efficient distributed schemes for embedding binary trees into incomplete hypercubes," Proceedings International Conference IEEE Region 10, Aug. 1994, vol. 1, pp. 182-186, Singapore.

[52] T.Y. Yen, W. Wolf, "Multiple-process behavioral synthesis for mixed hardwaresoftware systems", Proceedings $8^{\text {th }}$ International Symposium on System Synthesis, Sept. 1995, pp. 10-15, Cannes, France. 


\section{Appendix A - Prototype Device Code Listing}

The following VHDL and C code define the final prototype device described in section 4.2 and illustrated in figure 4.13. The device is a symmetrical three dimensional hypercube that performs the block matching algorithm.

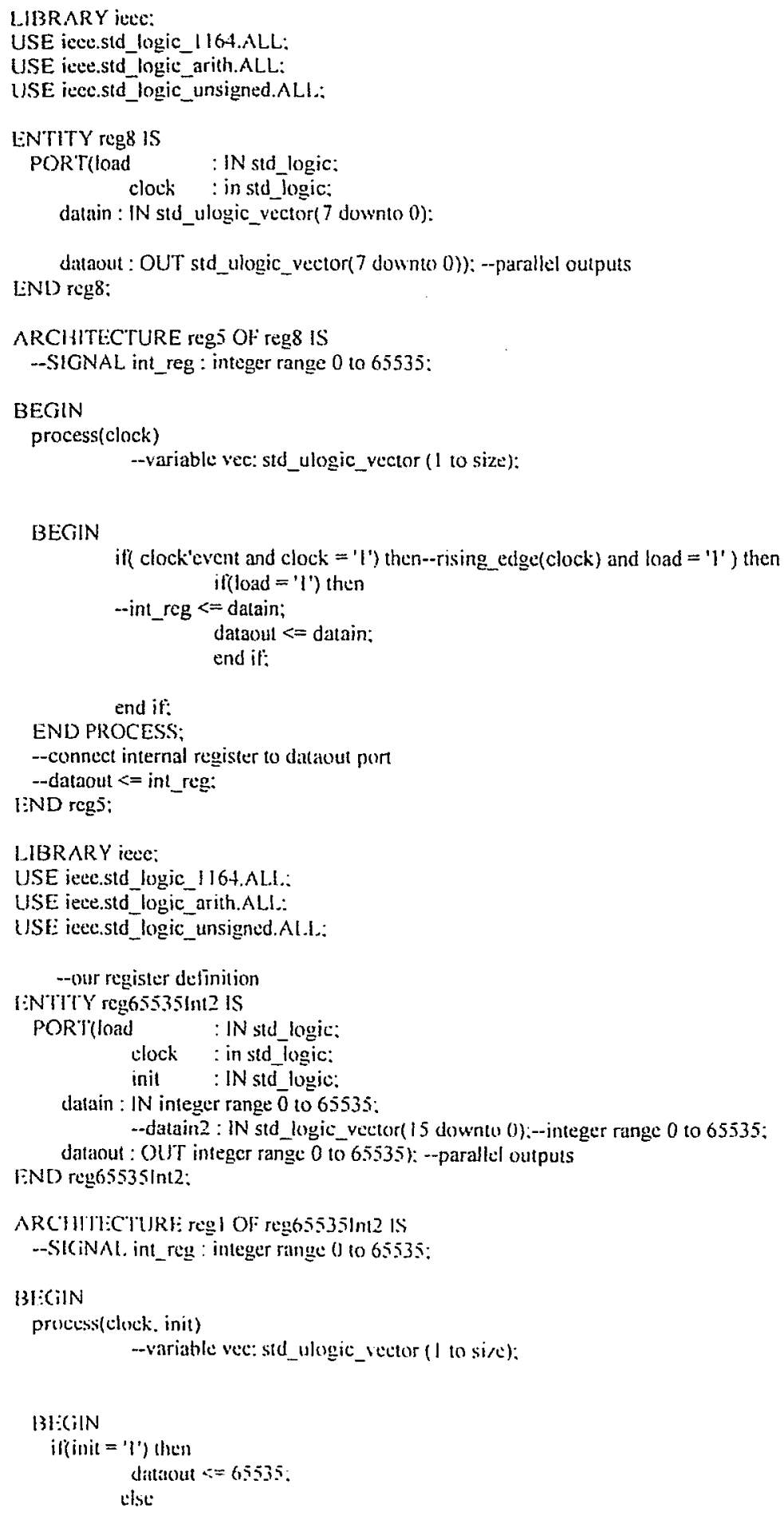




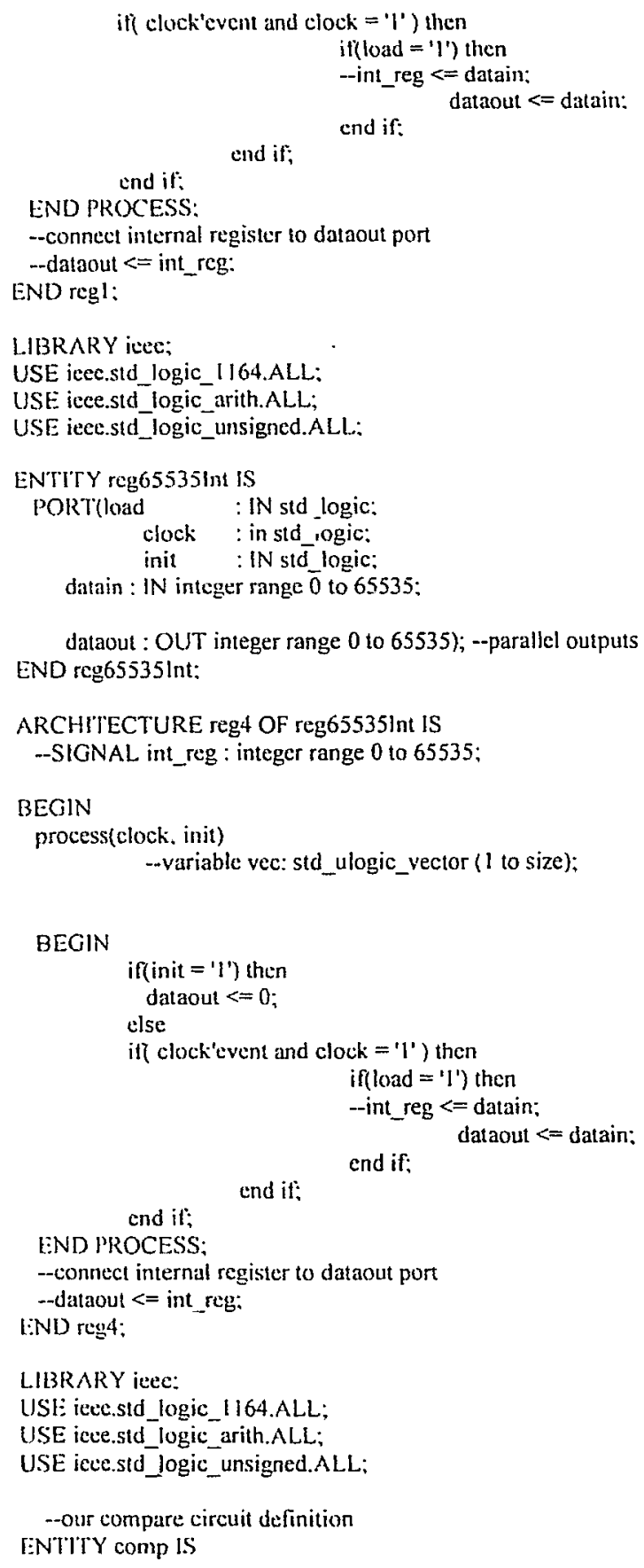


BEGIN

process(start)

begin

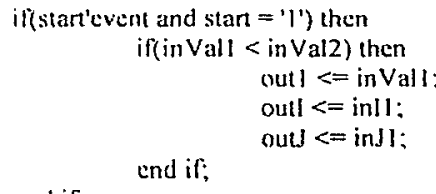

end if;

end process:

-oull < = outTemp;

END compl:

LIBRARY IEE:

USE icEe.std_logic_1164.Al.l:;

USE ieee.std_logic_arith.ALL:

USE icee.std_logic_unsigned.AL.L:

--our add circuit definition

ENTITY add IS

PORT(in1: IN integer range 0 to 65535: --paraltel inputs

in 2 : IN integer range 0 to 65535 : -parallel inputs

out : OUT integer range 0 to 65535): --parallel outputs

END add:

ARCHITECTURE addI OF add IS

signal outTemp : INTEGER;

BEGIN

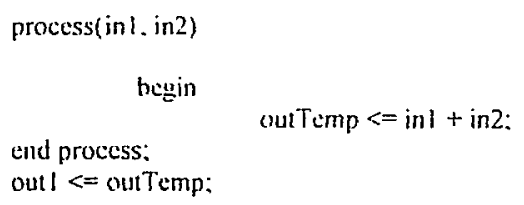

FND add:

LIBRARY IECE:

USI: ieet.std lugic 1164.Al.1:

USE iece.sId_logic_arith.ALL:

USE: iece.std_lngic_unsigned.All:

-our difference circuit delinition

ENTITY difTIS

POR7(inl: IN Sid_ulogic_vector(7 DOWNTO 0): --parallel inputs in2 : IN Stu_ulogic_vector(7 DOWNTO 0): -parallel inpuss l:NI) dill: out1 : OUT integer range $0-606535)$; --parallel outputs

ARCHITECTURI dIMI OH: diTI IS signal sut lemp : INTI:Cil:R:

B3I:CiIN

$$
\begin{aligned}
& \text { process(in) inn) } \\
& \text { variable val. INTTECil:R; } \\
& \text { variathle val! : INTECit:R } \\
& \text { variable val2 : INTECit:R: } \\
& \text { variathe b : INIt:cit:R: }
\end{aligned}
$$


BHECIN

$$
\begin{aligned}
& \text { val }:=0 \text {; } \\
& \text { b : }=1 \text {; } \\
& \text { for } y \text { in } 0 \text { to } 7 \text { loop } \\
& \text { if(inl }(y)=\text { 'l') then } \\
& \text { end if; } \\
& b:=b * 2 \text {; } \\
& \text { - end loop;--for y } \\
& \text { vall }:=\text { val; } \\
& \text {.val }:=0 \\
& \mathrm{~b}:=1 \text {; } \\
& \text { for } y \text { in } 0 \text { to } 7 \text { loop } \\
& \text { if } \left.\operatorname{in} 2(y)={ }^{\prime} l^{\prime}\right) \text { then } \\
& \text { val :=val }+\mathrm{b} \text {; } \\
& \text { end if; } \\
& b:=b * 2 \text {; } \\
& \text { end loop:--for } y \\
& \text { val2 }:=\text { val; } \\
& \text { if(vall < val2) then } \\
& \text { else } \\
& \text { out Temp }<=(\text { val2 }- \text { vall }) \text {; } \\
& \text { end if; } \\
& \text { out Temp }<=\text { (vall - val2); }
\end{aligned}
$$

END PROCESS;

out $1<$ out Temp;

END diffl:

I.IBRARY icec;

USE icet.std_logic_1164.ALL;

USE iee.std_logic_arith.ALL:

USE isce.std_logic_unsigned.ALL;

--our register detinition

ENTTTY reg IS

$$
\begin{gathered}
\text { PORT(load : IN sid_logic; } \\
\text { clock }
\end{gathered}
$$

datain : IN Sitd_ulogic_vector(7 DOWNTO 0); --parallel inputs

dataout : OUT Std_ulogic_vector(7 DOWNTO 0)); --parallel outputs IEND reg:

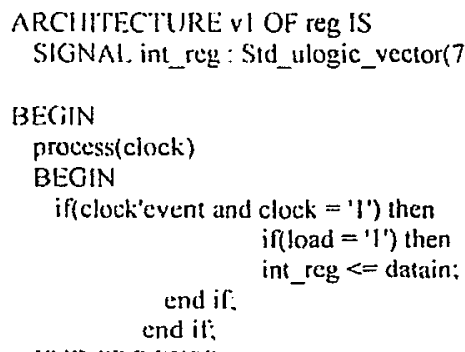

--connect internal register to dataout port

dalioul $<=$ int reg:-- when timeT'oOutput = 'l' else "ZZZZTZZZZ":

END VI:

L.JBRARY ICLE:

USH: iece.stl_logic _I 64.AI.L:

USt: iece.std hogic arith. ALL:

USE: iecc.std_legic_unsigned.ALL: 
ENTITY blockmatch IS

PORT

END blockmatch:

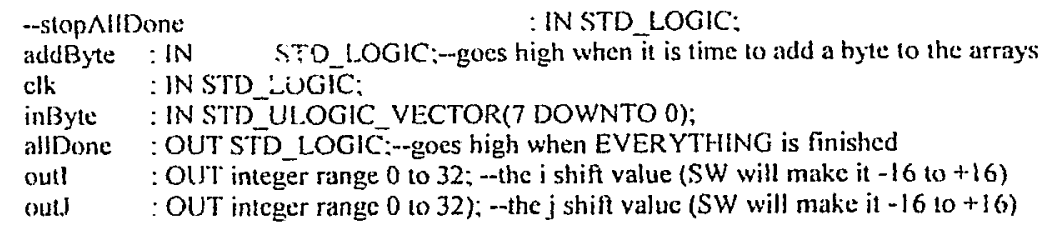

ARCHITECTURE description OF blockmatch IS

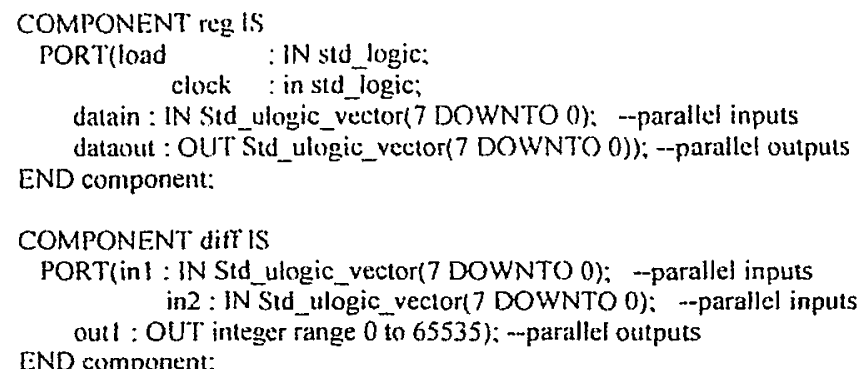

END component:

component add is

PORT(in : IN integer range 0 to 65535: --parallel inputs

in $2: 1 \mathrm{~N}$ inleger range 0 to 65535; - -parallel inputs

outl : OUT inleger range 0 to 65535 ); --parallel outputs

END component:

coinponent comp IS

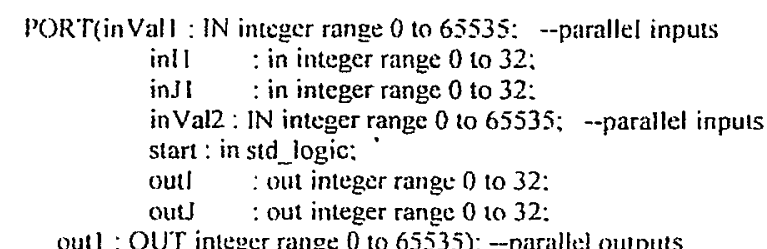

I:ND component:

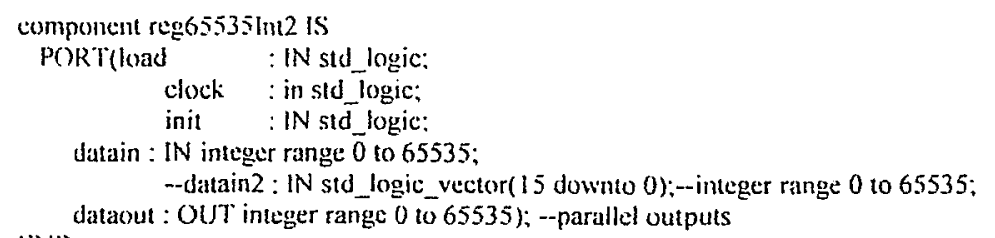

ENI component:

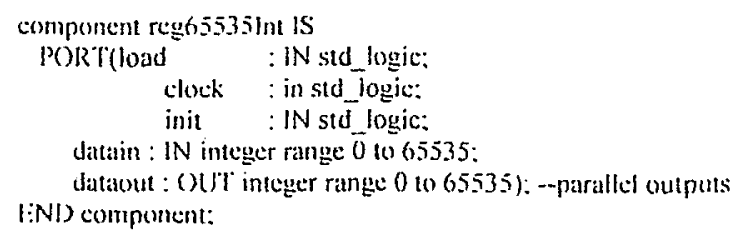

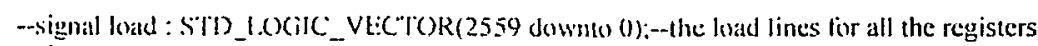

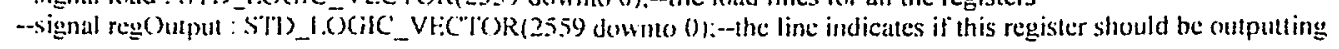


signal regActiveOut : STD_ULOGIC_VECTOR(7 downto 0):

--signal regOldOu : STD ULOGIC_VECTOR(7 downto 0);

signal diffl Out : integer range 0 to 65535 ;

signal addRegOut : integer range 0 to 65535 ;

--signal luad2ndReg : STD_LOGIC_VECTOR(1088 downto 0);

--signal reg2ndOutput: STD_LOGIC__VECTOR(1088 downto 0);

signal iVal : integer range 0 to 32 ; --generated by control circuitry to tell conparator what index is being compared

signal jVal : integer range 0 to $32 ;-$ as above

signal compOut : integer range 0 to 65535 ;

signal iOut: integer range 0 to 32 ;

signal jout: integer range 0 to 32 ;

signal bestError Yet : integer range 0 to 65535 ;

signal loadCompReg : STD_LOGIC:

signal inilCompReg: STD_LOGIC:

-signal regAdderln2 : integer range 0 to 65535 ;

--signal regActiveTimeToOutput : std_logic_vector(255 downto 0);

--signal regOldTimeToOutput : std_logic_vector(2303 downto 0);

signal addOut : integer range 0 to 65535 :

signal loadAddReg : std logic;

signal initAdaReg: std_logic:

signal loadinReg : std_Jogic;

signal reg2ndOut : std_logic_vector( 15 downto 0 );

--subtype WORD8 is STD_LOGIC_VECTOR (7 downto 0);

-type ARRAY 1089 is array ( 1088 downto 0) of WORD8;

-signal reg2ndOut : ARRAY 1089 ;

signal compStart : std_Jogic;

BEGIN

inRegActiveLabel: reg PORT MAP(loadinReg, clk, inBytc, regActiveOut):

dit7Circuit: diff port map(regActiveOut, inByte, difflOut);

add I: add port map(diffiout, addRegOut, addOut):

addRegLabel: reg65535Int port map(loadAddReg, clk, initAddReg, addOut, addRegOut):

compl: comp port map(addOut, iVal, jVal, bestErrorYet, compStart, iOut, jOut, compOut);

compRegister: reg65535Int2 port map(loadCompReg, clk, initCompReg. compOut, bestError Yet):

--templnRegOut $<=$ regActiveOut:

-tempDiffOut $<$ dimOut;

-tempAddRegOut $<=$ addRegOut:

--tempAddOut $<=$ addOut:

-tempCompRegOut <= bestErrorYet;

-tempCompOut $\quad<=$ compOut;

process(clk)

variable addCounter : integer;

variable compCounter: integer;

variable $\mathrm{i}$ : integer;

variable $\mathrm{j}$ : integer;

the adding

$$
\text { variable state }
$$

: integer : $=0 ;-0$ means we are loading values. I means we are doing

bcgin

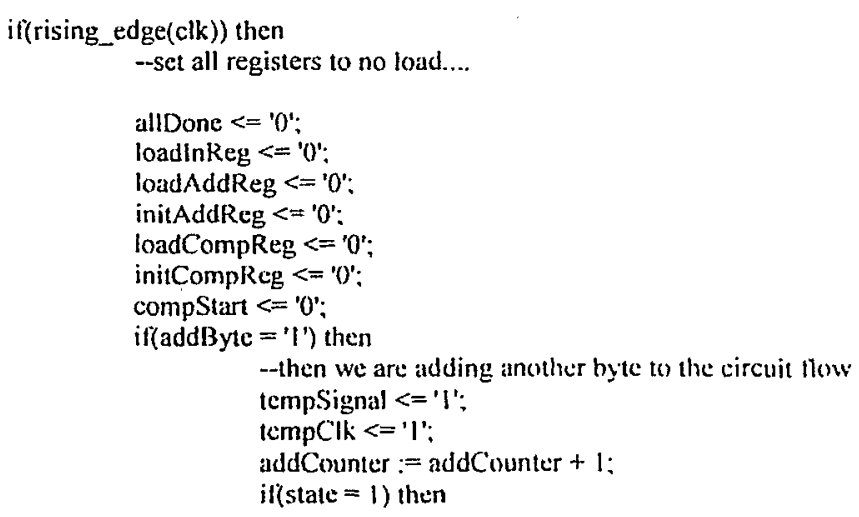




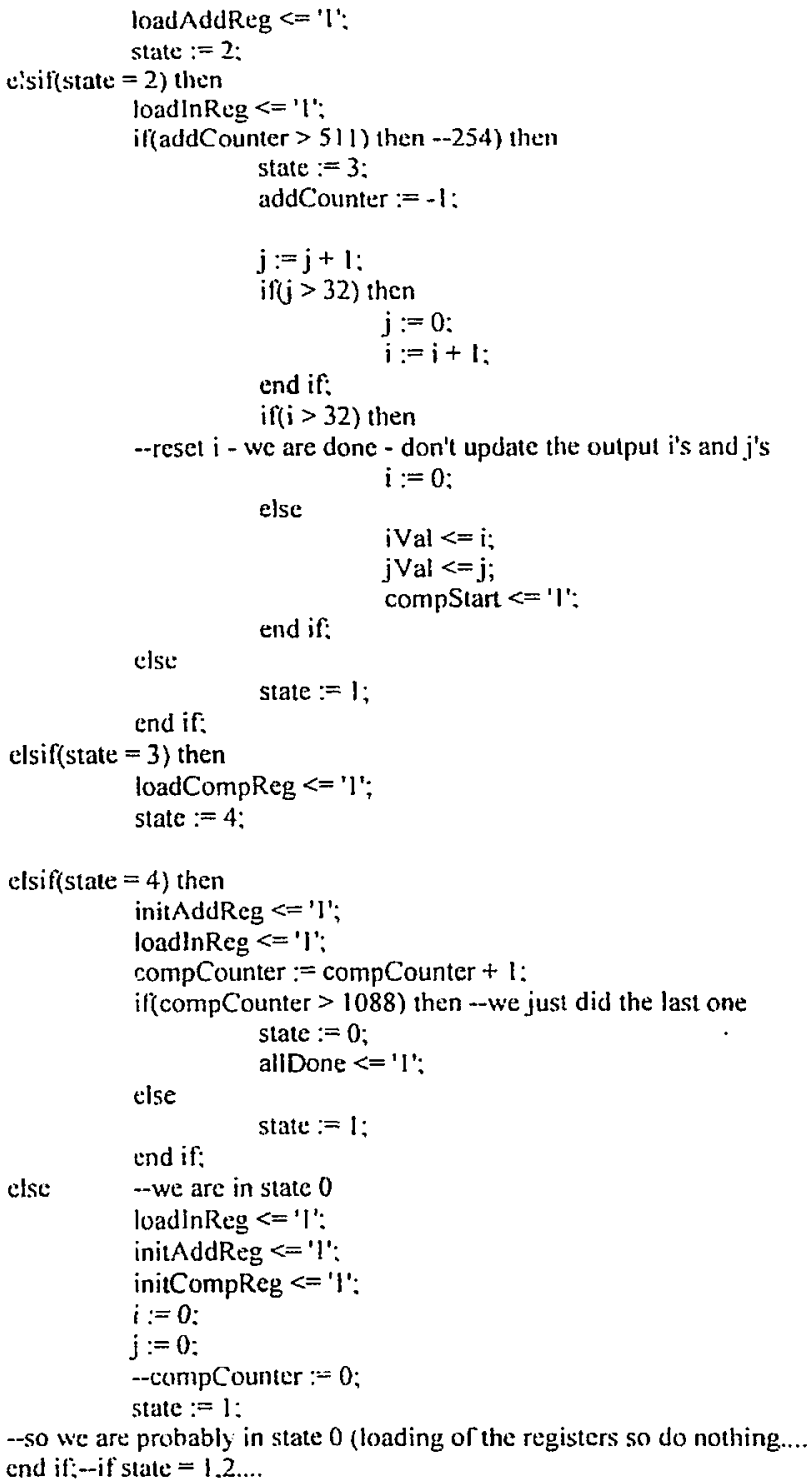

F:ND description:

I.HBRARY iete:

USF: ievestd logic 1164. Al.L:

USI: ices:std logic arith.Al.1.

USE ices.std_logic unsignted.Al.l:

E:NITTY PI: BlockMatch IS

PORTI

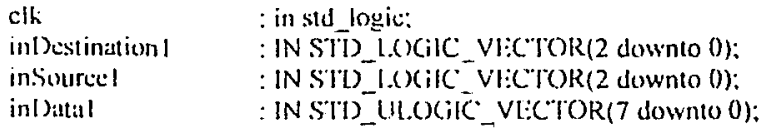




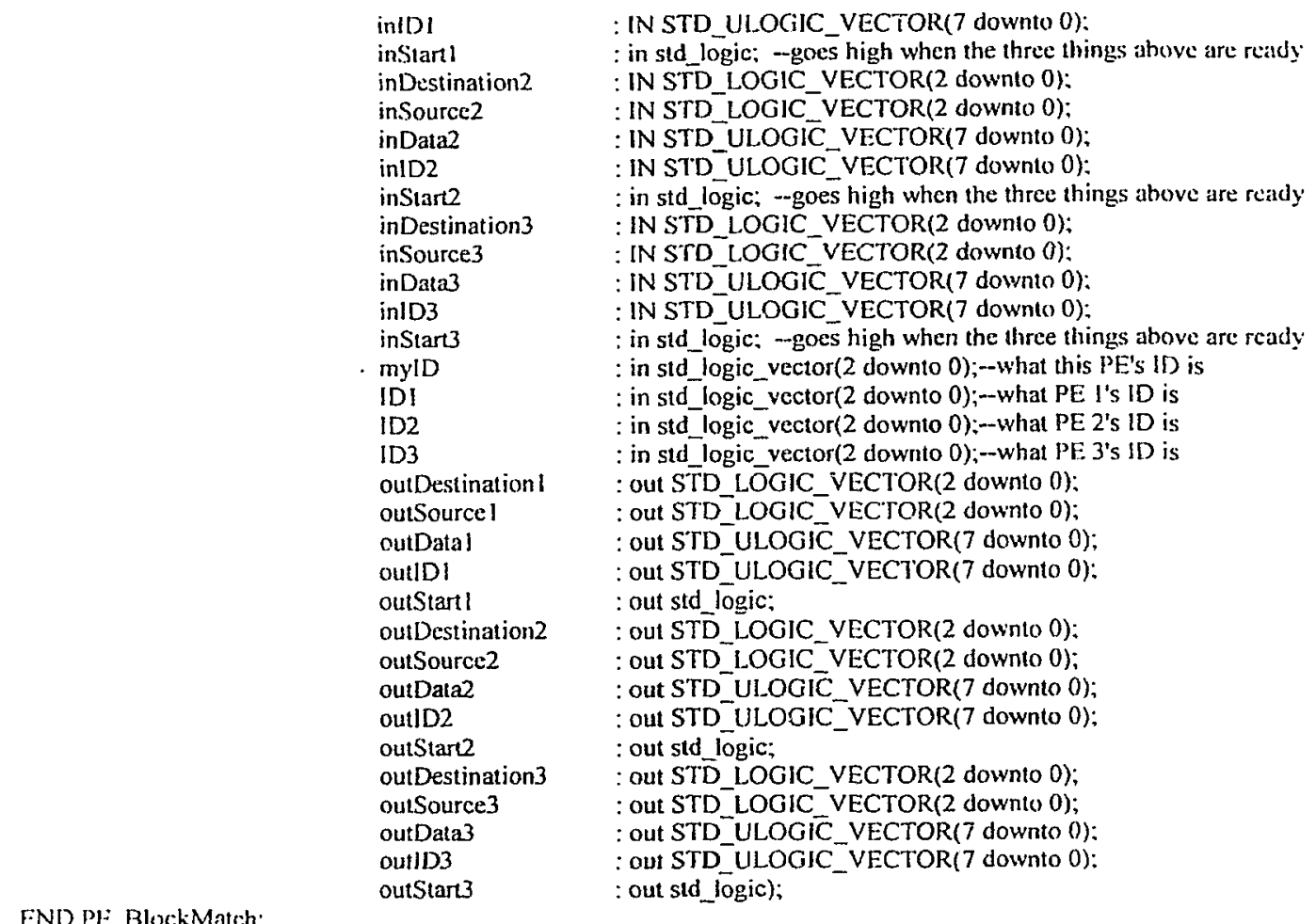

ARCHITECTURE PEdescription OF PE_BlockMatch IS

component blockmatch IS

PORT(

addByte : IN

clk :IN STD LOGIC:

inByte :IN STD ULOGIC VECTOR(7 DOWNTO 0);

alIDone : OUT STD LOGIC;-goes high when EVER YTHIING is finished

outl :OUT integer range 0 to 32 ; - the i shift value ( $S W$ will make it $-1610+16$ )

END component;

out : OUT integer range 0 to 32 ); --the $j$ shift value ( $S W$ will make it $-1610+16$ )

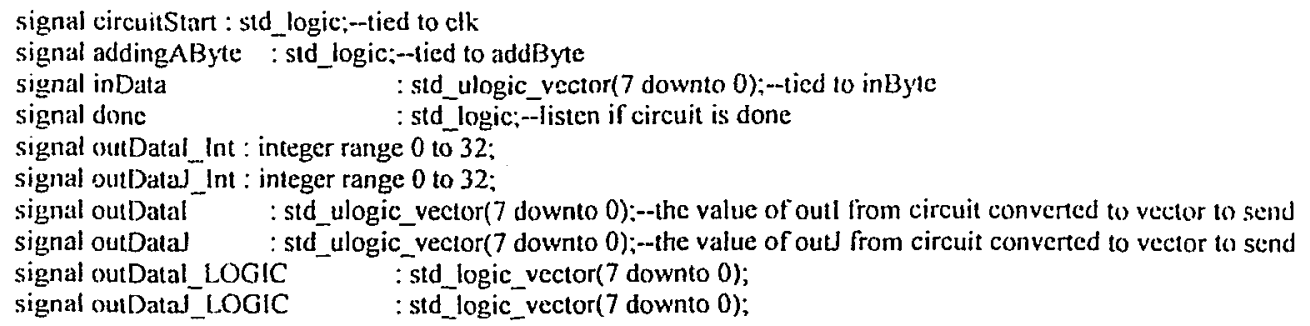

BM: blockmatch port map(addingAByte, clk, inData, done, outDatal_Int, out1)atid_Int):

$\operatorname{process}(\mathrm{clk})$

variable destCounterl : integer range 0 to 3 ; variable destCounter2: integer range 0 to 3 ; variable destCounter 3 : integer range 0 to 3 :

variable iTemp : integer range 0 to 32 : 


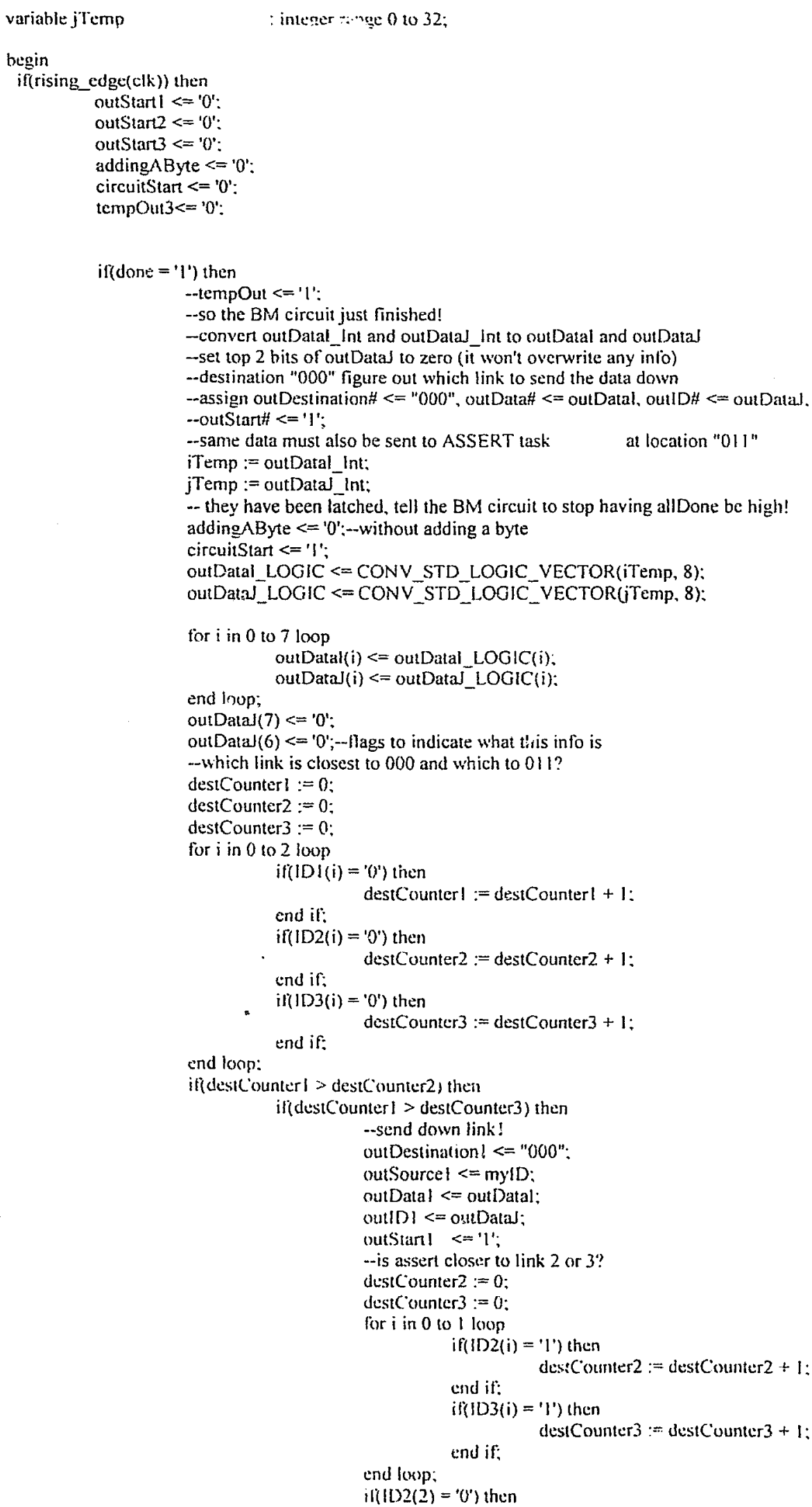

113 


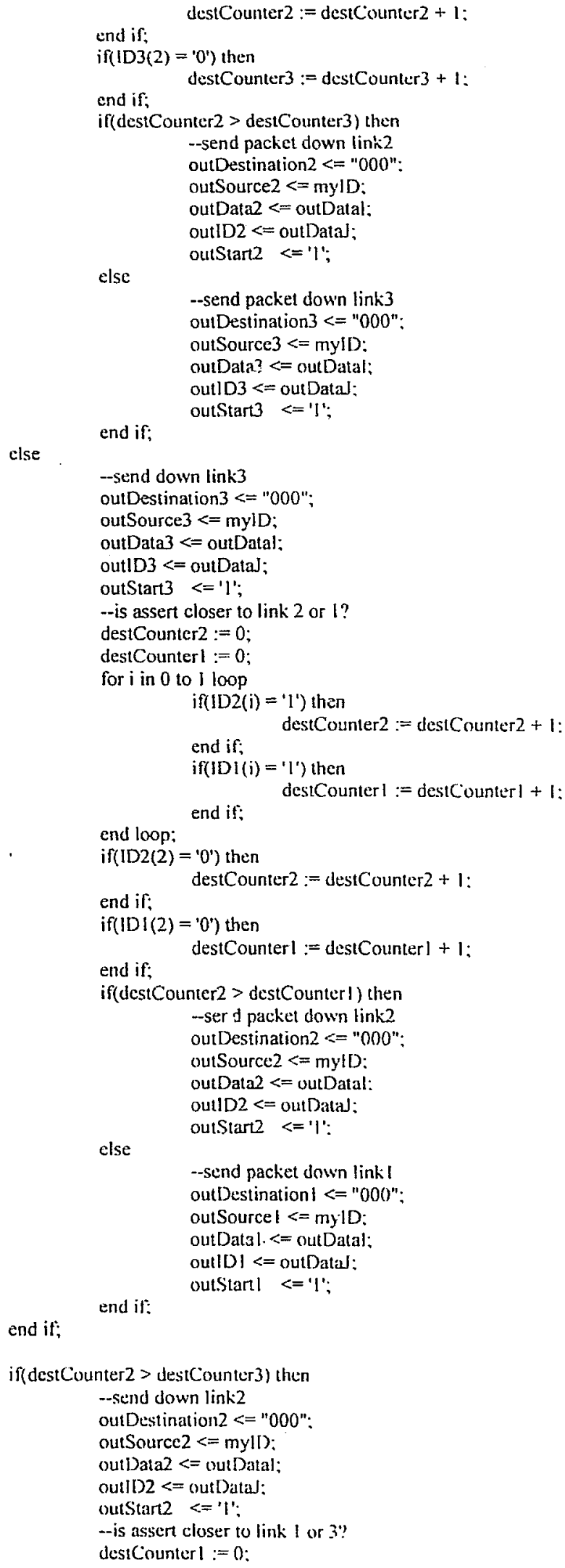




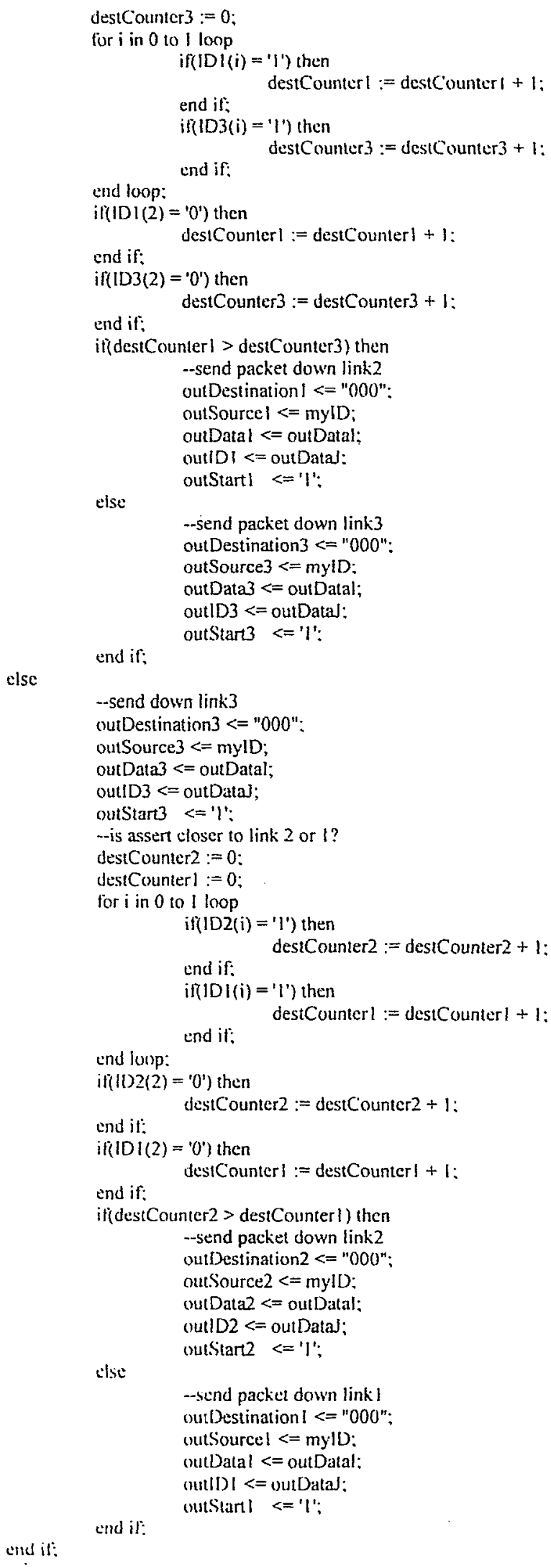

end it: 


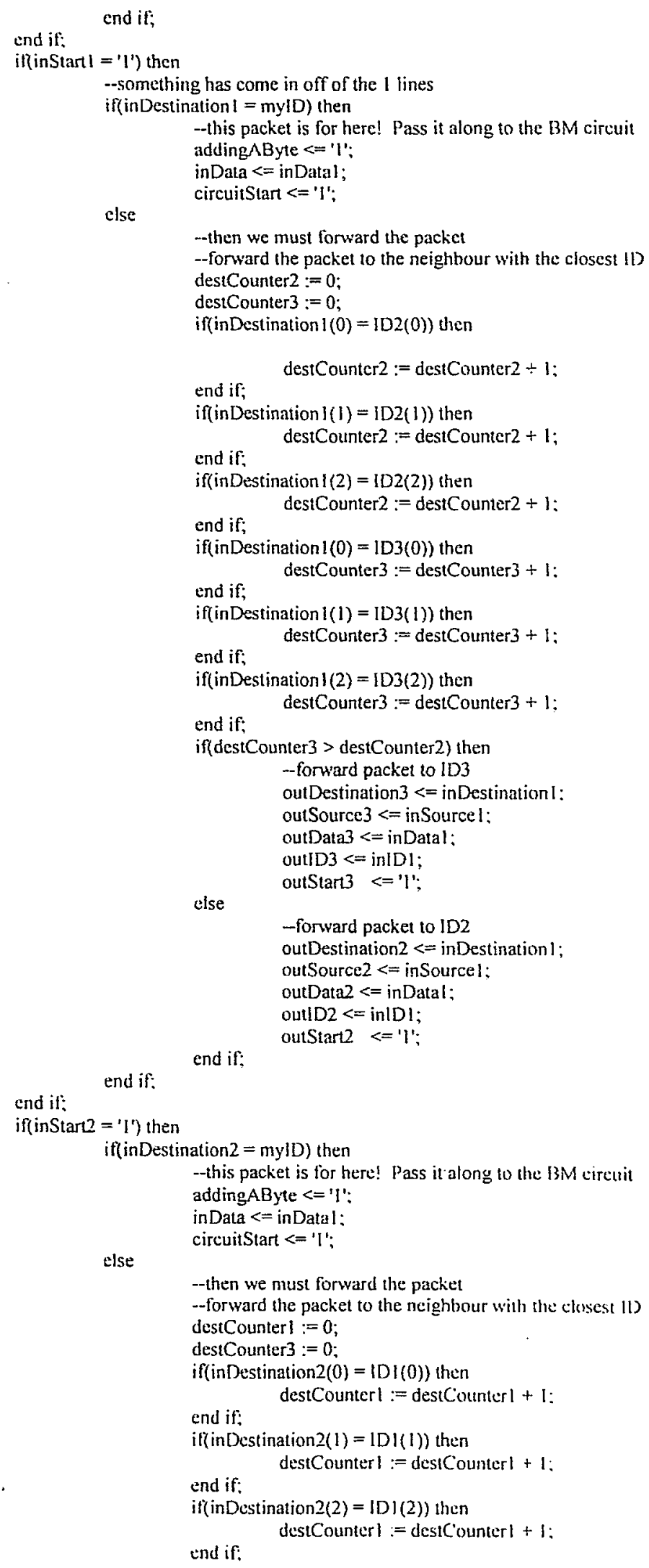




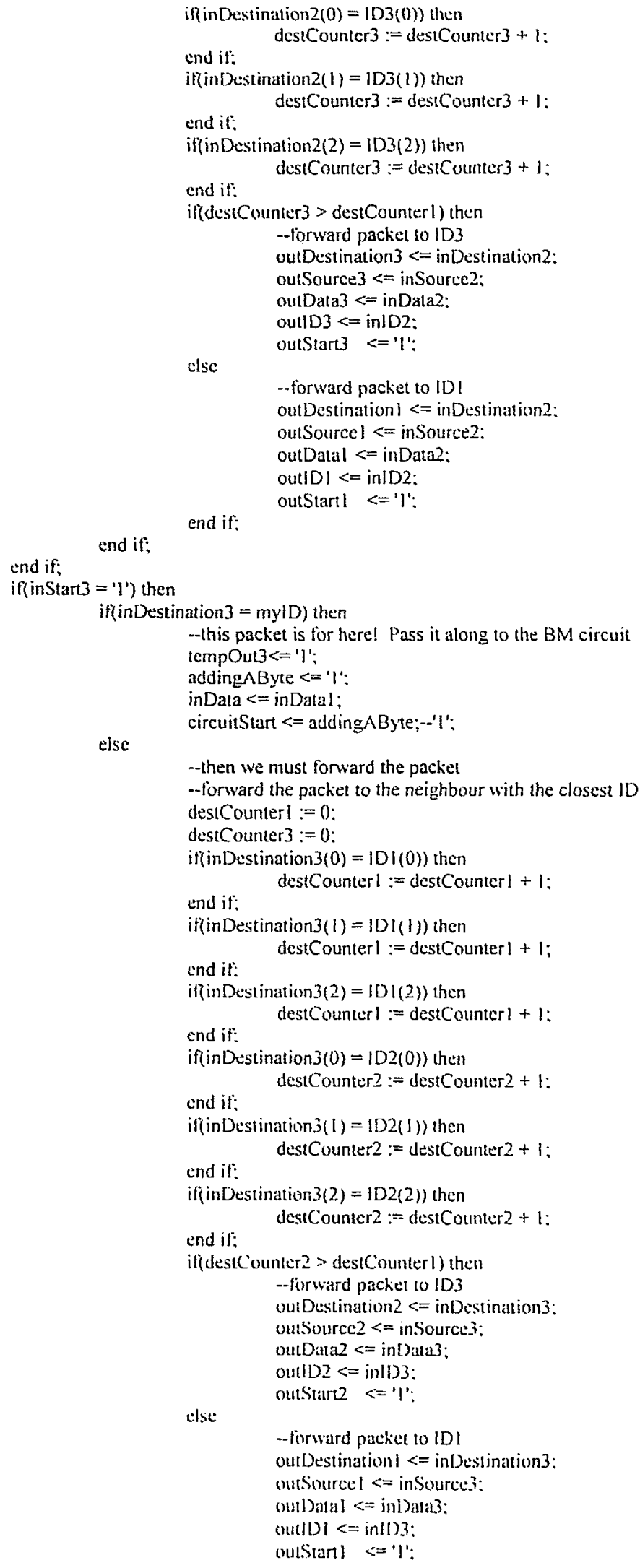


end if;

end if:

end if:

end process:

end PEdescription:

end if;

LIBRARY itee:

USE iece.std_logic_1164.ALL:

USE icce.sid_logic_arith.ALL:

USE ieer.std_logic_unsigned.ALL;

ENTITY PE_BM_Asser IS

PORTI

\begin{tabular}{|c|c|}
\hline clk & in std logic; \\
\hline inDestination 1 & : IN STD_LOGIC_VECTOR(2 downto 0): \\
\hline inSourcel & : IN STD_LOGIC_VECTOR(2 downto 0): \\
\hline inDatal & : IN STD_ULOGIC_VECTOR(7 downto 0$)$; \\
\hline inIDI & : IN STD_ULOGIC VECTOR (7 downto 0): \\
\hline Start! & in std_logic; -goes high when the three things above are ready \\
\hline Destination 2 & :IN STD_LOGIC_VECTOR(2 downto 0); \\
\hline ISource2 & :IN STD_LOGIC_VECTOR(2 downto 0$)$; \\
\hline Data2 & :IN STD_ULOGIC_VECTOR $(7$ downto 0$)$ \\
\hline inID2 & : IN STD ULOGIC VECTOR(7 downto 0$)$; \\
\hline instart2 & : in std logic; -goes high when the three things above are ready \\
\hline inDestination 3 & : IN STD_LOGK_VECTOR(2 downto 0); \\
\hline inSource3 & :IN STD_LOGIC_VECTOR(2 downto 0); \\
\hline inData3 & :IN STD_ULOGIC_VECTOR $(7$ downto 0$)$ : \\
\hline inID3 & : IN STD ULOGIC VECTOR(7 downto 0 ); \\
\hline inStart3 & : in std_logic: -goes high when the three things above are ready \\
\hline mylD & : in std_logic_vector(2 downto 0);--what this PE's ID is \\
\hline IDI & : in std_logic_vector $(2$ downto 0$)$;--what PE I's ID is \\
\hline ID2 & : in std_logic_vector(2 downto 0);--what PE 2's ID is \\
\hline & : in std_logic_vector(2 downto 0 );--what PE 3's ID is \\
\hline outDestinat & : out STD_LOGIC_VECTOR(2 downto 0): \\
\hline outSource I & : out STD_LOGIC_VECTOR(2 downto 0); \\
\hline outDatal & : out STD_ULOGIC_VECTOR(7 downto 0): \\
\hline outlDi & :out STD_ULOGIC_VECTOR(7 downto 0): \\
\hline outStart I & : out std_logic: \\
\hline Destination 2 & : out STD_LOGIC_VECTOR(2 downto 0): \\
\hline Source 2 & : out STD LOGIC VECTOR( 2 downto 0$)$; \\
\hline outData2 & : out STD_ULOGiC_VECTOR(7 downto 0): \\
\hline outlD2 & : out STD_ULOGIC_VECTOR(7 downio 0); \\
\hline outStarl2 & : out std_logic: \\
\hline outDestinati & : out STD_LOGIC_VECTOR(2 downto 0): \\
\hline outSource3 & : out STD_LOGIC_VECTOR(2 downto 0): \\
\hline outData3 & : out STD_ULOGIC_VECTOR(7 downto 0 ): \\
\hline IDD3 & : out STD_ULOGIC_VECTOR(7 downto (0): \\
\hline WtStart3 & out std_logic); \\
\hline
\end{tabular}

ENDPE_BM_Assert:

outStart

ARCHITECTURE PEAsseridescription OF PE_BM_Assert IS

begin

process(clk)

variable destCounterl : integer range 0 to 3 :

variable desiCounter 2 : integer range 0 to 3 :

variable desiCounter 3 : integer range 0 to 3 ;

variable sendt:rror : integer range 0 to 1 ;--yoss high if error is delected (so a packet must be sert to the --control so that that PE is now neglected)

variahlc hadlBM : std_logic_vector(2 downto 0);--represents which $13 \mathrm{M}$ is taulty

begin

iffrising edge(c)k ) then outStartl <= '0': 


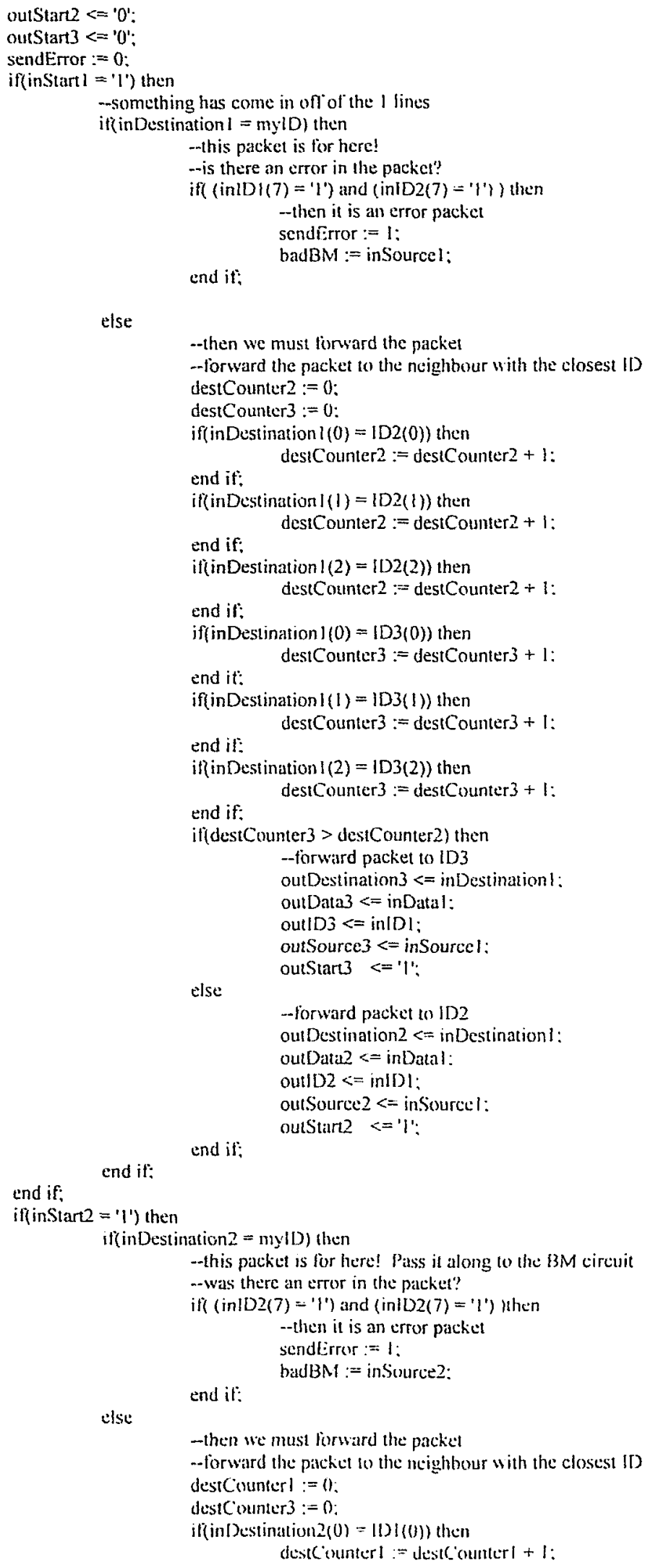

else

-then we must forward the packet

--Forward the packet on the neighbour with the closest ID destCounicer : $=0$ :

destcounter $3:=0$

il (inD Destination $2(0)=\mid 1) \mid(0))$ then

destcenterl : $=$ destcounterl +1 . 


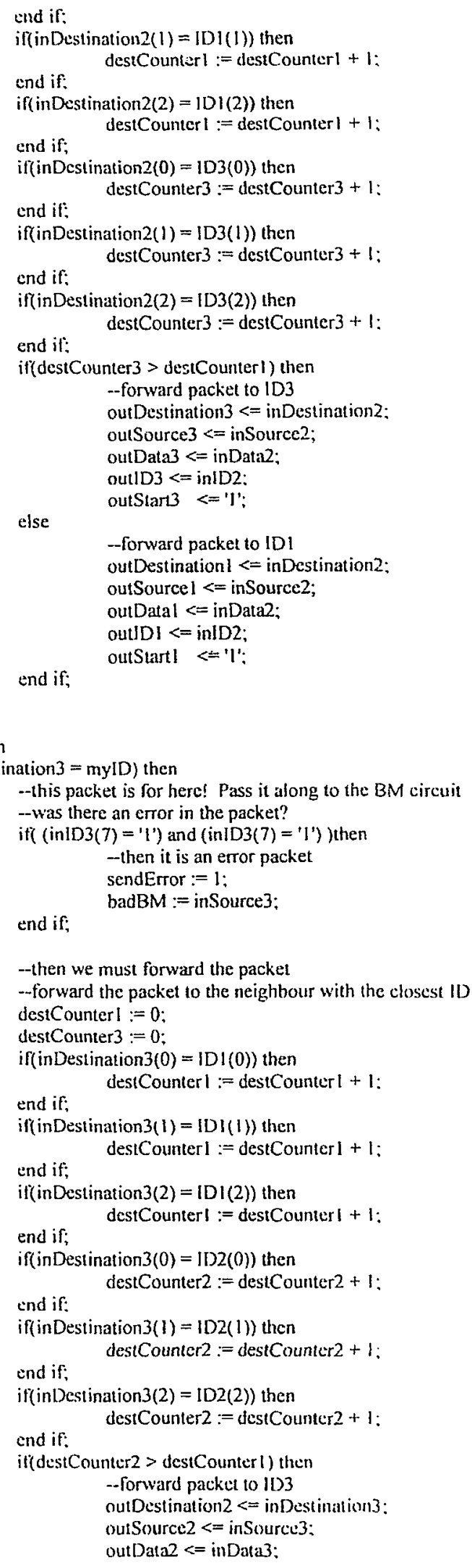




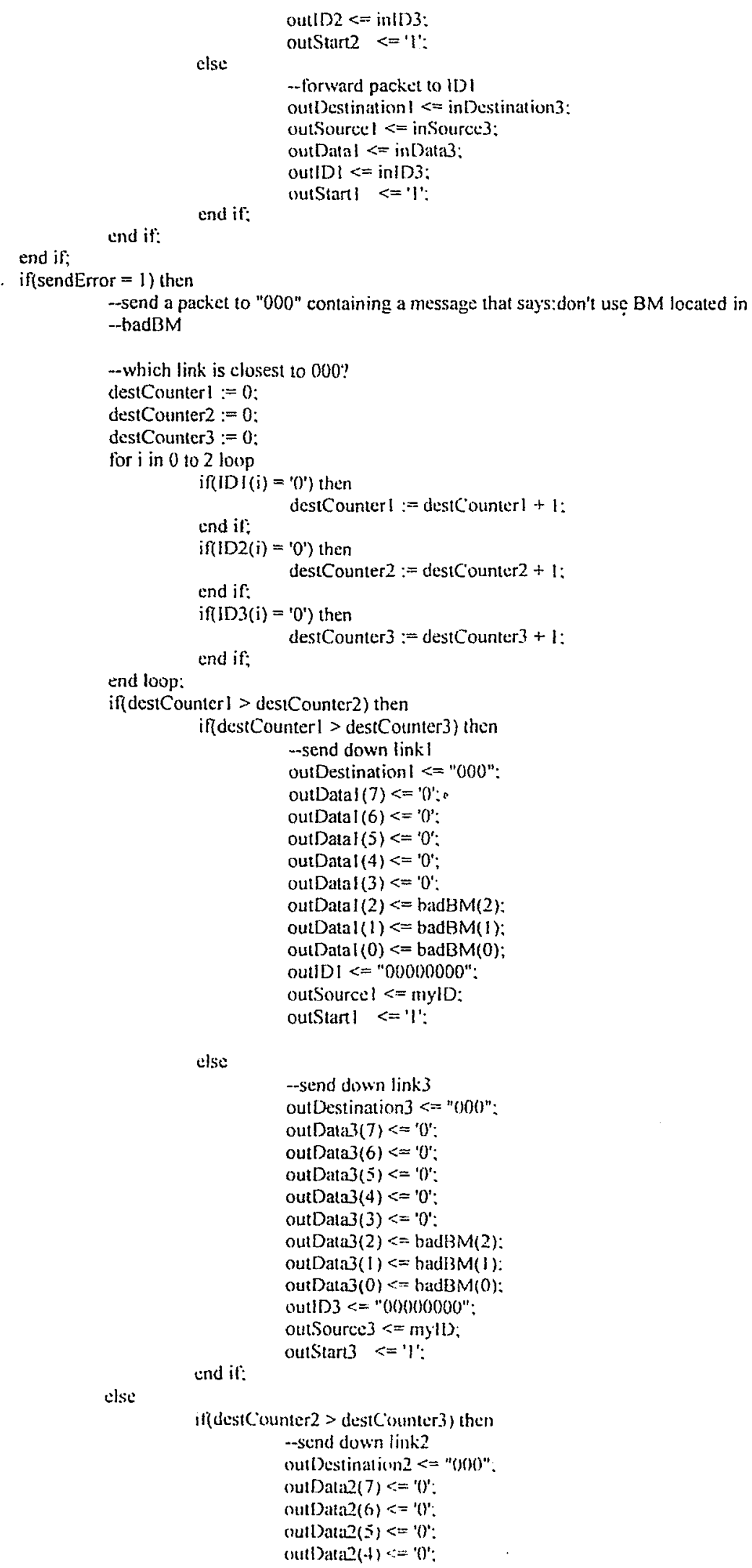




\begin{tabular}{|c|}
\hline $\begin{array}{l}\text { outData2 }(3)<=\text { '0' } \\
\text { outData2 }(2)<=\text { badBM }(2) \text {; } \\
\text { outData2 }(1)<=\text { badBM }(1) \text {; } \\
\text { outData2 }(0)<=\text { badBM }(0) \\
\text { outID2 }<=\text { "00000000": } \\
\text { outSource2 }<=\text { myID: } \\
\text { outStart }<=1 \text { : }\end{array}$ \\
\hline 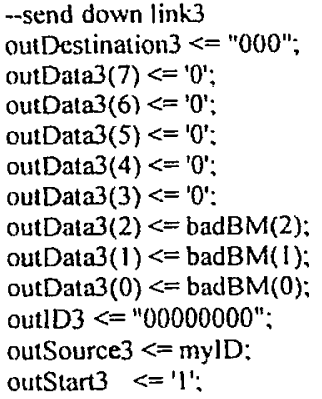 \\
\hline
\end{tabular}

end if:

end PEAssertdescription;

end process;

LIBRARY iee:

USE iceestd_logic 1164.ALL:

USE ieee.sid logic_arith.ALL;

USE iceesstd_logic_unsigned.ALL;

ENTITY PE_Final_Asser IS

PORT.

\begin{tabular}{|c|c|}
\hline clk & in std_logic; \\
\hline inDestination 1 & IN STD_LOGIC_VECTOR(2 downto 0$)$ : \\
\hline inSourcel & IN STD_LOGIC_VECTOR $(2$ downto 0$)$ : \\
\hline inDatal & IN STD_ULOGIC_VECTOR(7 downto 0); \\
\hline iniD! & : IN STD_ULOGIC_VECTOR(7 downto 0); \\
\hline inStart & in std logic; -goes high when the three things above are ready \\
\hline inDestination 2 & IN STD_LOGIC_VECTOR(2 downto 0): \\
\hline inSource 2 & : IN STD_LOGIC_VECTOR(2 downto 0): \\
\hline inData2 & : IN STD_ULOGIC_VECTOR(7 downto 0): \\
\hline inlD2 & IN STD_ULOGIC_VECTOR(7 downto 0$)$ : \\
\hline inStart2 & in std_logic; --goes high when the three things above are ready \\
\hline inDestination 3 & : IN STD LOGIC VECTOR(2 downto 0$)$ : \\
\hline inSource3 & IN STD_LOGIC_VECTOR (2 downto 0); \\
\hline inData3 & IN STD_ULOGIC_ VECTOR(7 downto 0): \\
\hline inID3 & :IN STD_ULOGIC_VECTOR(7 downto 0); \\
\hline instart3 & in std logic; --goes high when the three things above are ready \\
\hline mylD & in std logic vector (2 downto 0$)$; - what this PE's ID is \\
\hline IDI & in std_logic_vector(2 downto 0 );--what PE I's ID is \\
\hline ID2 & in std_logic_vector( 2 downto 0$)$; --what PE 2's ID is \\
\hline ID3 & in std_logic_vector(2 downto 0$)$;--what PE 3 's ID is \\
\hline outDestination 1 & : out STD LOOGIC_VECTOR(2 downto (0): \\
\hline outSourcel & : out STD_LOGIC_VECTOR(2 downto 0); \\
\hline outDatal & out STD_ULOGIC_VECTOR(7 downto 0): \\
\hline outlD 1 & out STD_ULOGIC_VECTOR(7 downto (0): \\
\hline outStart 1 & out std_logic; \\
\hline outDestination? & out STD_LOGIC_VECTOR(2 downto 0): \\
\hline outSource? & out STD_LOGIC_VECTOR(2 downto 0); \\
\hline outData2 & out STD_ULOGIC_VECTOR(7 downto 0): \\
\hline OutID2 2 & : out STD ULOGIC_VECTOR(7 downto 0): \\
\hline outStart2 & out sid_logic: \\
\hline outDestination3 & out STD_LOGIC_VECTOR(2 downto 0): \\
\hline outSource3 3 & out STD_LOGIC_VECTOR(2 downto 0): \\
\hline outData3 & out STD_ULOGIC_VECTOR(7 downto 0): \\
\hline
\end{tabular}


FND PE_Final_Assert:

$\begin{array}{ll}\text { outlD3 } & \text { : out STD_ULOGIC_VECTOR }(7 \text { downto } 0): \\ \text { outStart3 } & \text { : out std_Fgic): }\end{array}$

ARCHITECTURE PEFinalAssertdescription OF PE_Final_Assen IS

begin

process(clk)

variable destCounter 1 : integer range 0 to 3 ;

variable destCounter 3 : integer range 0 to 3 :

variable sendErtor : integer range 0 to 1 ;--gocs high if error is detected (so a packet must be sent to the -control so that that $P E$ is now neglected)

variable badBM : std logic vector(2 downto 0$)$;--represents which $B M$ is faulty

begin

if(rising_edge(clk)) then

outStartl $<=$ ' 0 ':

outStart2 $<=$ ' 0 ':

outStart3 $<=$ ' 0 ';

sendError $:=0$;

if(inStart $\left.1={ }^{\prime}{ }^{\prime}\right)$ then

--something has come in off of the 1 lines

if(inDestination $1=$ mylD) then

-this packet is tor here!

-is there an error in the packet?

if $($ inIDI $(7)=1$ ') and $($ inID2 $(7)=11))$ then

-then it is an error packet

sendError :=1;

end if;

badBM := inSourcel ;

else

-then we must forward the packet

-forward the packet to the neighbour with the closest ID

destCounter $2:=0$ :

destCounter $3:=0$ :

if $($ inDestination $1(0)=1 D 2(0))$ then

destCounter $2:=$ destCounter $2+1$ :

end if:

if $($ inDestination $/(1)=11) 2(1))$ then

destCounter2:= destCounter2 +1 :

end it:

if (inDestination $(\underline{2})=11 \mathrm{I} 2(2))$ then

destCounter $2:=$ destCounter $2+1:$

end if:

if (inl)estination $l(0)=1103(0))$ then

end if:

destCounter $3:=\operatorname{destCounter} 3+1$ :

if(inDestination $1(1)=1 \mathrm{D} 3(1))$ then

destCounter $3:=\operatorname{destCounter} 3+1$ :

end if:

iff(inDestination $(\{2)=1 \mathrm{D} 3(2))$ then

destCounter $3:=$ destCounter $3+1$;

end if:

iff(destCounter $3>$ destCounter2) then

-forsvard packet to ID3

outDestination $3<=$ inl)estinationl:

outData $3<=$ infatal:

outli) $3<=$ inll)!:

(nutSource $3<=$ in.Source]:

ilse

outstart3 <= '1';

-forward packes to ID2

oul Destination $2<:=\mathrm{jul}$ )estination l:

(utl)aul $<=$ inlatal: 


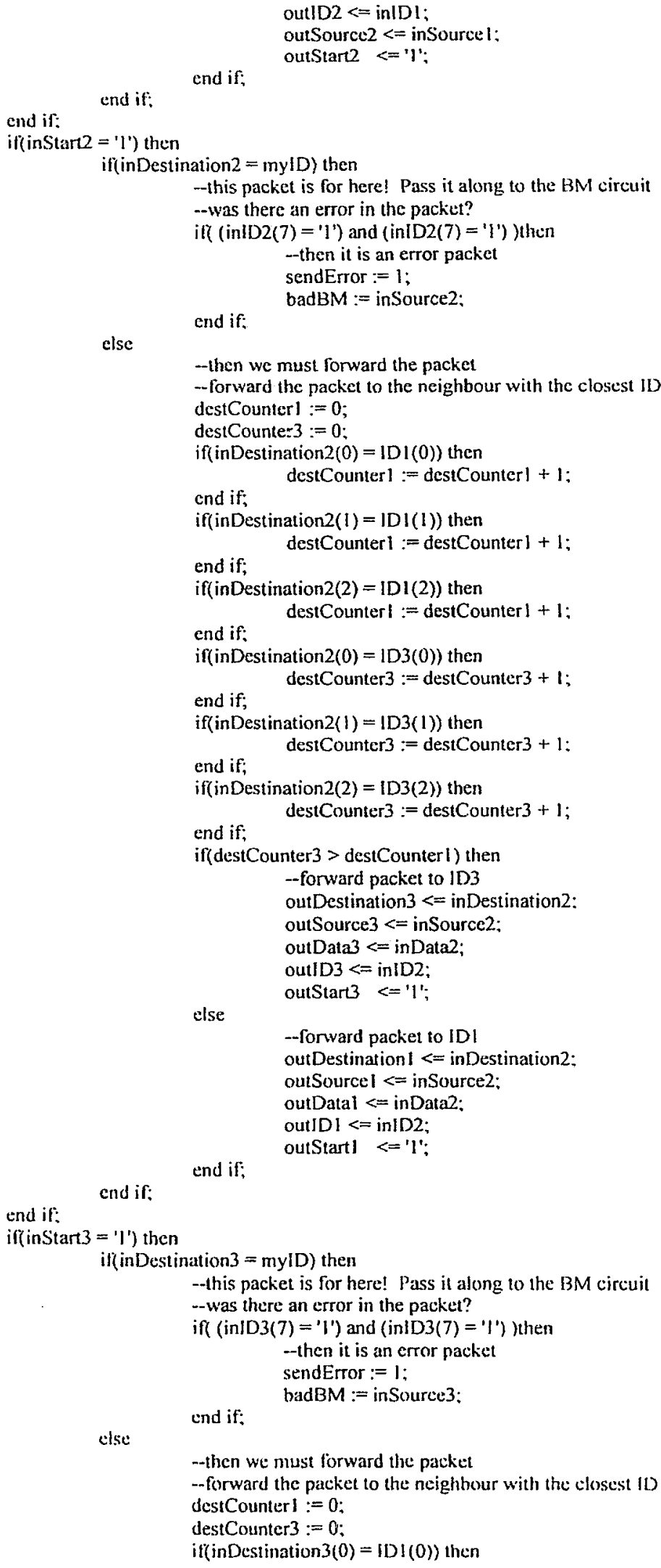


end if:

destCounterl := destCounterl +1 :

if(inDestination $3(1)=1 \mathrm{D} \mid(1))$ then

destCounterl:= destCounter +1 :

end if:

if $($ inDestination $3(2)=|D|(2))$ then

destCounterl $:=$ destCounterl +1 :

end if:

if (inDestination $3(0)=[D 2(0))$ then

destCounter $2:=\operatorname{destCounter} 2+1$;

end if:

if (inDestination $3(1)=102(1)$ ) then

destCounter $2:=$ destCounter $2+1$;

end if:

if ( inDestination $3(2)=1 D 2(2))$ then

destCounter $2:=\operatorname{destCounter} 2+1$ :

end if:

if(destCounter $2>\operatorname{destCounter~} 1)$ then

-forward packet to ID3

outDestination $2<=$ inDestination 3 ;

outSouree 2 < = inSource3:

outData2 $<=$ inData 3 :

outlD2 $<=$ inlD3:

else

outStart2 <= 'I':

-forward packet to IDI outDestination $1<=$ inDestination3; outSourcel $<=$ inSource3;

outDatal $<=$ inData3:

outiDI $<=$ inID3;

end if:

outStarl <='l'.

end if:

end if;

if(sendError $=1)$ then

-send a packet to "000" containing a message that says:don't use BM located in -badBM

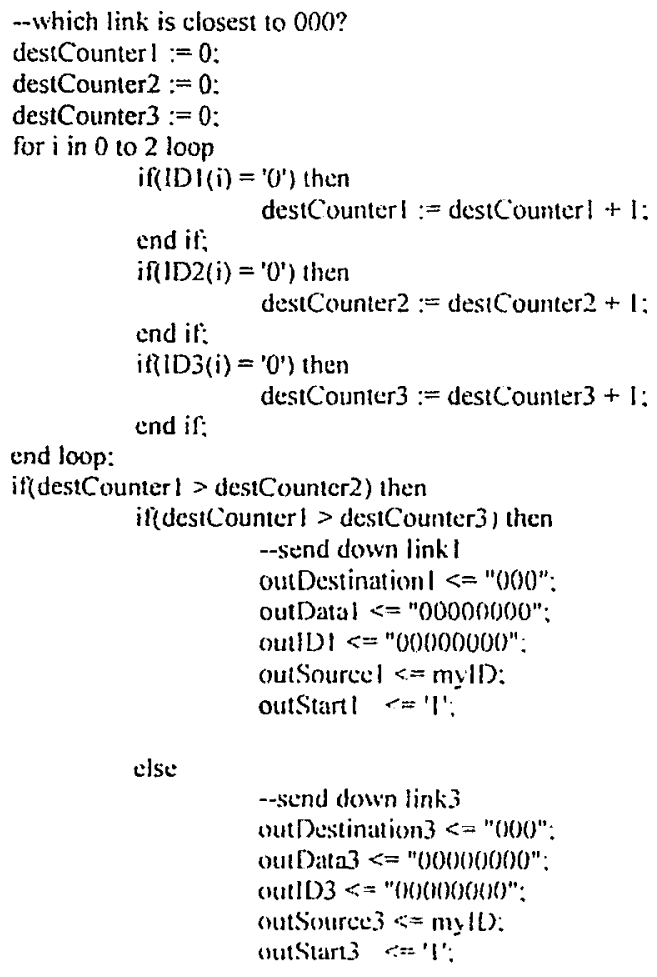


outData3 : out SID_ULOGIC_VECTOR(7 downto 0):

outID3 : out STD_ULOGIC_VECTOR\{7 downto 0):

-lemp temp

tempBMCounter : out integer range 0 to 13;

tempDestinationBM : out std_logic_vector(2 dow nto 0):

END PE_CPU:

outStar3

: out sld_logic):

\section{ARCHITECTURE PECPU OF PE_CPU IS}

Component reg8 IS

PORT(load

: IN std_logic;

clock : in std logic;

datain : IN std_ulogic_vector(7 downto 0):

dataout : OUT std_ulogic_vector(7 downto 0)); --parallel outputs

END component:

signal loadiReg: std_logic_vector(13 downto 0);

signal loadJReg : std_logic_vector( 13 downto 0$)$ :

signal BMlntermediatel : std ulogic yector $(7$ downto 0$)$ :

signal BMIntermediated : std_ulogic_vector(7 downto 0);

subtype WORD8 is STD_ULOGIC_VECTOR (7 downto 0):

type ARRAYOFBYTESI4 is array (13 downto 0) of WORD8:

signal BMIData : ARRAYOFBYTES 14;

signal BMiData : ARRAYOFBYTES 14;

-signal BMIDestination : std_logic_vector(2 downto 0$):=$ "001";

-signal BM2Destination : std logic vector $(2$ downto 0$):=" 010 "$ :

-signal SpareBM! : std_logic_vector $(2$ downto 0$):=101 "$ ":

--signal SpareBM2 : std_logic_vector $(2$ downto 0$):=110^{n:}$

-signal SpareBM3 : std_logic_vector $(2$ downto 0$):=111 "$ ":

--signal destinationBM : std_logic_vector(2 downto 0$):=001$ ";

-signal BMcounter : integer range 0 to $13:=0$;

begin

--reg l: for $\mathrm{i}$ in 0 to 13 generate

-- $\quad$ reglLabel: reg8 port map(loadIReg(i). clk, BMintermediatel. BMIData(i)):

- $\quad$ reg2Labei: reg8 port map(loadJReg(i). clk. BMllntermediateJ. BMJData(i)):

--end generate;

process $(\mathrm{clk})$

variable destCounter $1:$ integer range 0 to 3 :

variable destCounter 2 : integer range 0 to 3 :

variable destCounter 3 : inleger range 0 to 3 ;

variable $B M c o u n t e r$ : integer range 0 to $13:=0$;

variable BMIDestination : std_logic_vectur(2 downto 0$):=$ "001":

variable BM2Destination : std logic vector $(2$ downto 0$):=$ "0 010 ".

variable SpareBM1 : std_logic_vector $(2$ downto 0$):=" 101 "$ :

variable SpareBM2 : std logic vector $(2$ downto 0$):=" 11()^{\prime}$ :

variable SpareBM3 : std_logic_vector(2 downto 0$):=" 111 "$ ",

variable destination BM : sid_logic_vector $(2$ downto 0$):=001 "$ ":

variable BMOutCounter : integer : $=0$ :

variable packet roGo : integer range 0 to $1:=0$ :

variable toGoDestination : std logic vector(2 downto 0):

variable tocioData : std_ulogic_vector(7 downtw ()):

variable to Golink: integer range 1 to 3 :

begin

tempDestinationBM $<=$ destination $3 \mathrm{M}$ :

templBMCounter $<=$ BMCounter;

if(rising_edge $(\mathrm{c} \mid k))$ then

outstart! $<=$ ' 0 ',

outStant $<=$ ' 0 ',

outStan3 $<=$ ' 0 ': 


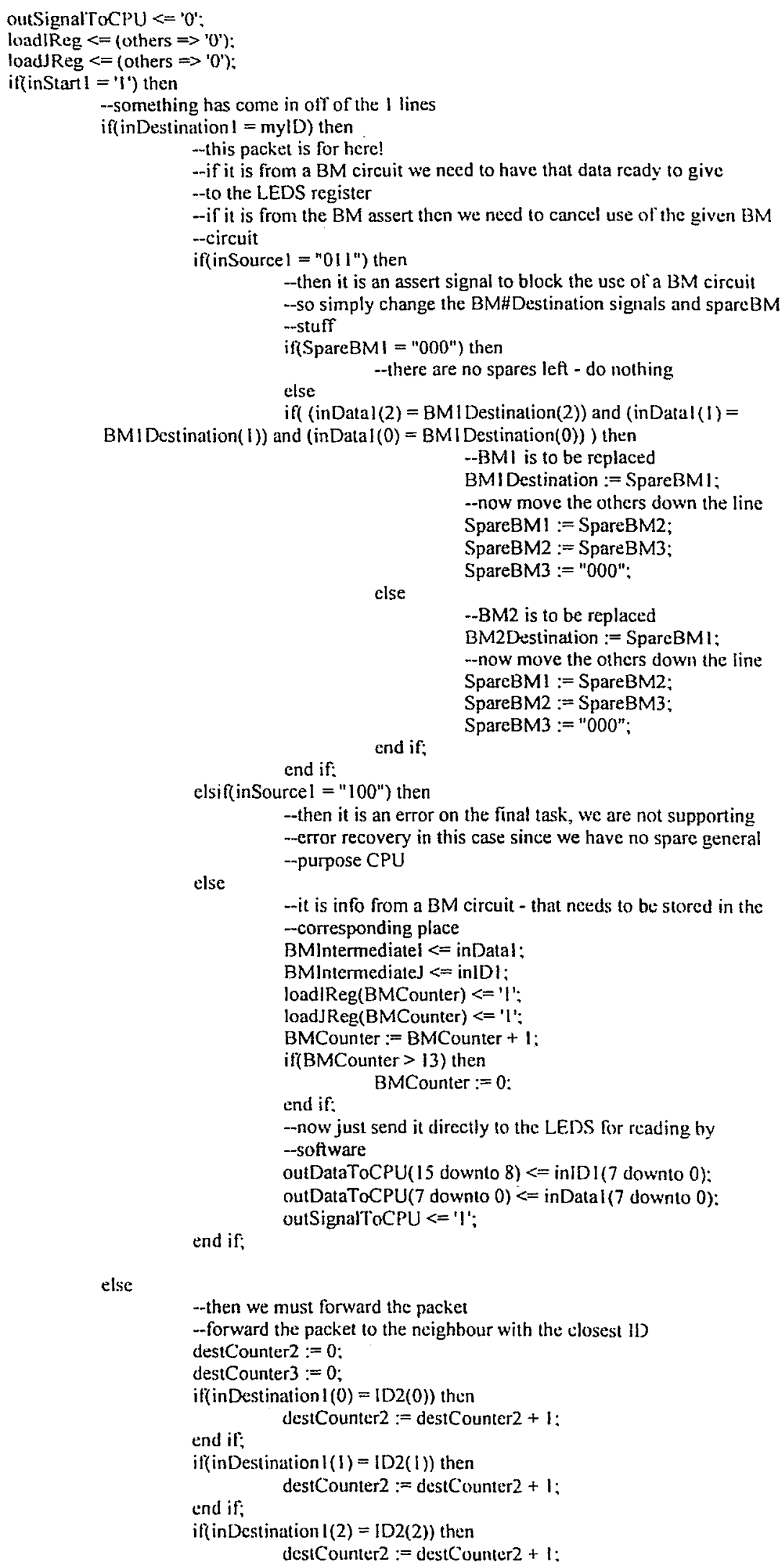

else 


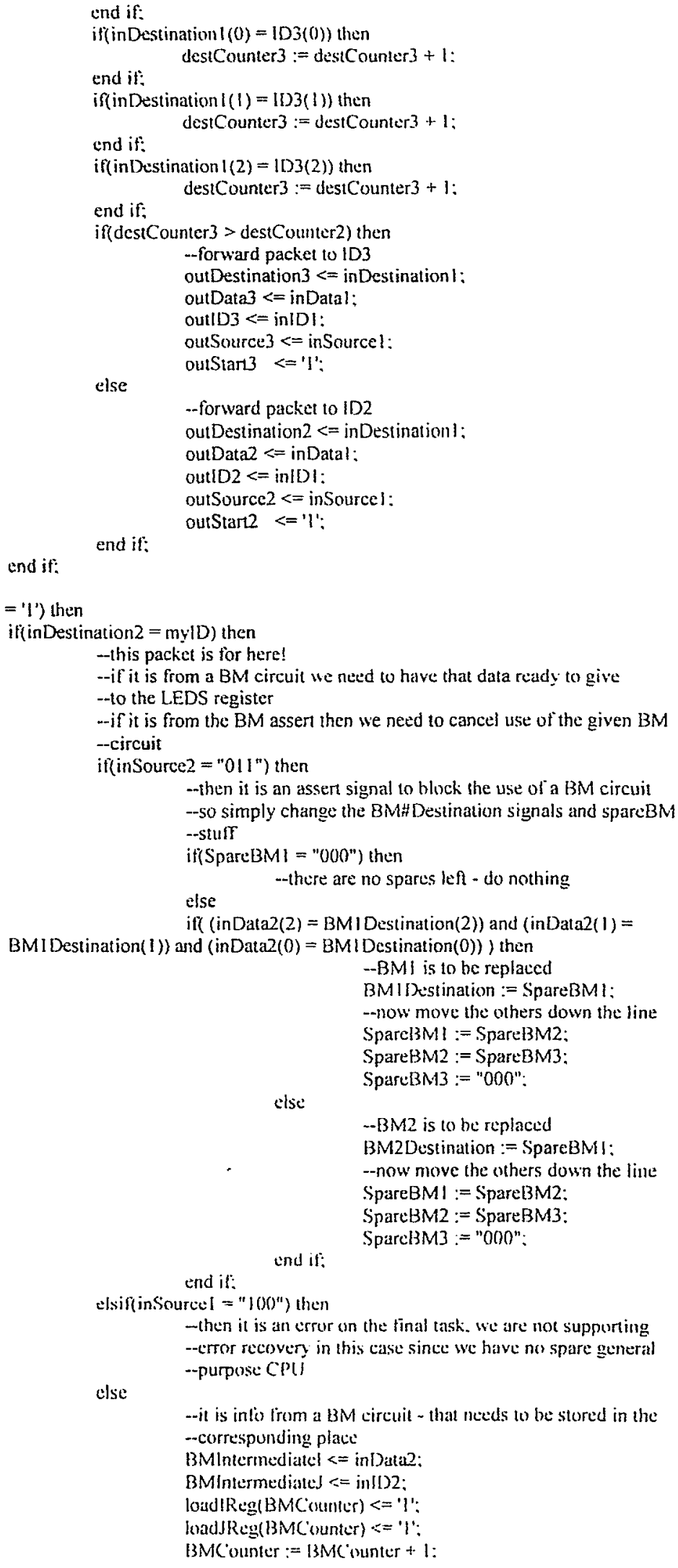




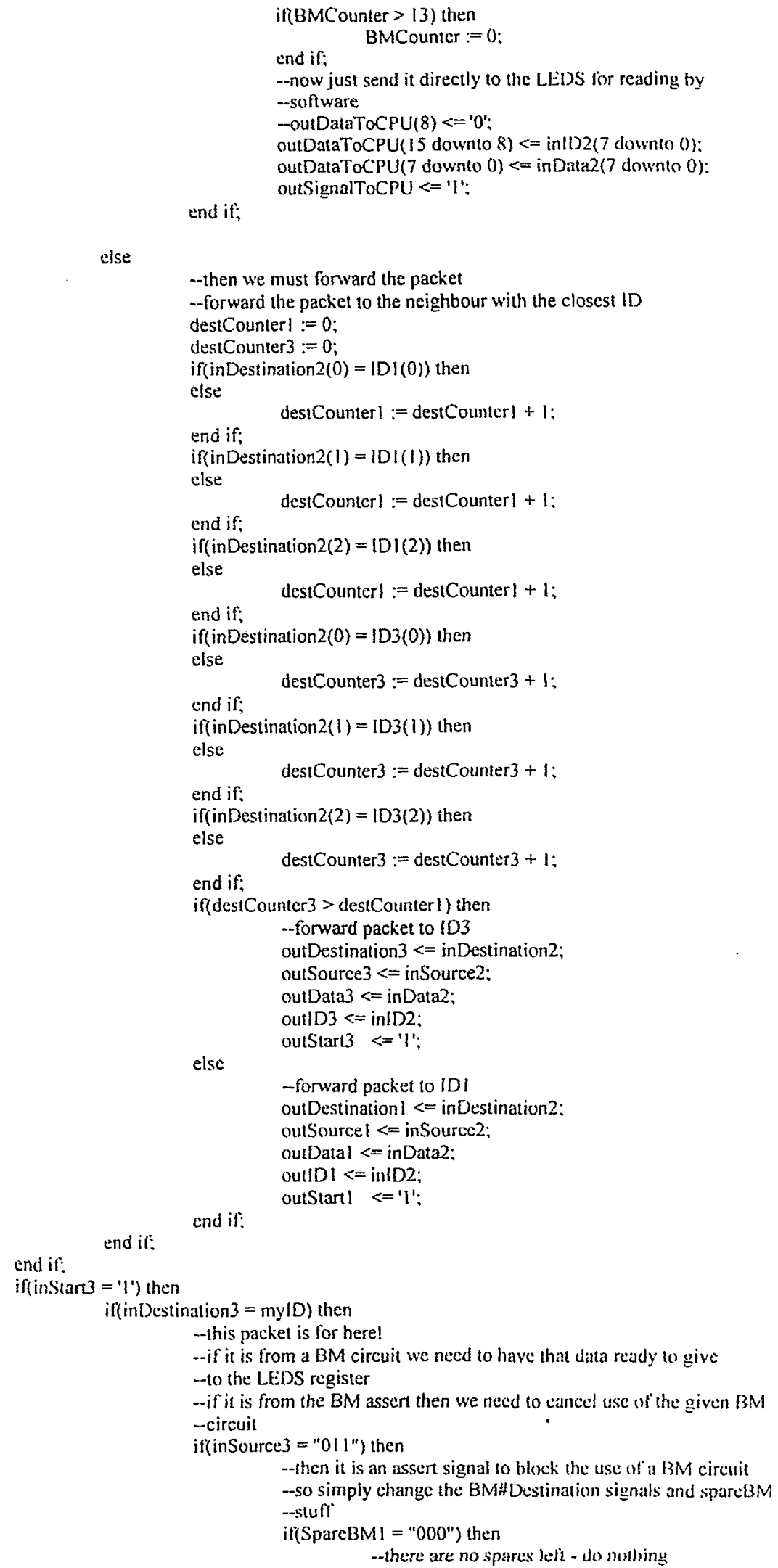


else

iff (inData3(2) $=$ BMIDestination(2)) and

(inData3(1) = BMIDestination(1)) and (inData3(0) $=$ BMIDestination(0)) ) then

-BMI is to be replaced

BMIDestination : $=$ Sparcl3Mt:

- now muve the others down the line

SparcBMI : $=$ SparcBM2;

SparcBM2 := SpareBM 3

clse

SpareBM3 := "(100":

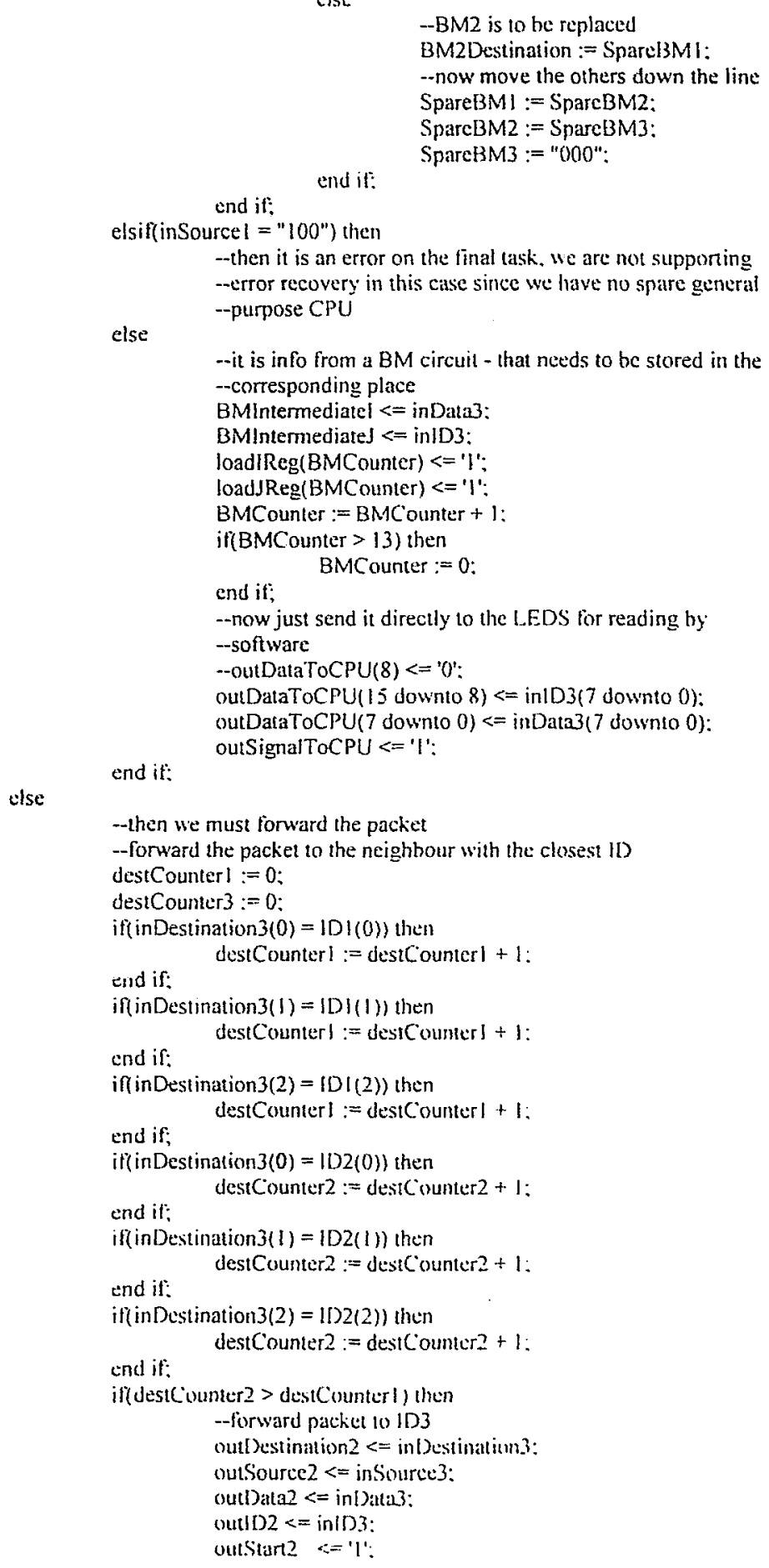


else

--forward packel to IDI

outDestination $1<=$ in Destination3;

oulSourcel $<=$ inSource 3 .

outDatal <= inData3;

outlDI <= inlo3;

end if:

outStart $<=' 1$ ':

end if;

end if;

if(inSignalFromCPU $=$ 'l') then

--then there is incoming data from the CPU it needs to be distributed to a BM -circuit

- or if it is a signalling packut we need to put info on the LEDS for reading... if (inDataFromCPU $(16)=$ ' 1 ') then

-then it is signalling and we should load the contents of the register -indexed

--in the lower 7 bits to the LEDS

if (inDatafromCPU $(15)=$ ' 1 ') then if(inDataFromCPU $(14)=$ ' 1 ') then

-signal from CPU to change the BM destination -circuit!

if (SpareBMI = "000") then

-there are no spares left - do nothing

else

if (inDataFrom $C P U(2)=$

BMIDestination(2)) and (inDataFromCPU(1) =

BMIDestination ( $(1)$ ) and (inDatafromCPU $(0)=$ BMIDestination(0)) ) then

-BMI is to be replaced BMIDestination := SpareBMl: -now move the others --down the line SpareBM1 := SpareBM2; SpareBM2 := SpareBN43;

clse SpareBM3 := "000":

--BM2 is to be replaced BM2Destination := SpareBM1: -now move the others --down the line SpareBM1 := SpareBM2 SpareBM2:= SpareBM3:

end if:

SpareBM3 := "000"

end if:

end if:

else

end if:

-outSignalToCPU $<=$ ' 1 '.

elsc

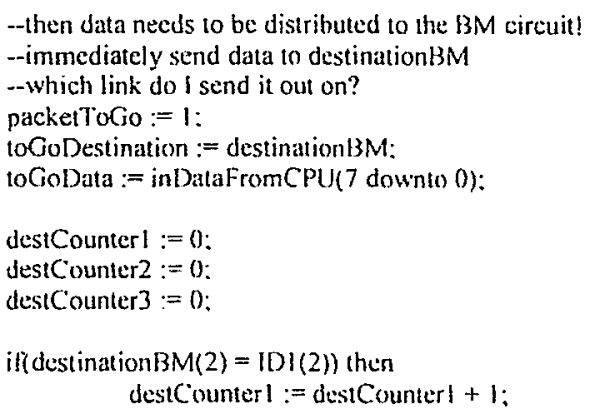




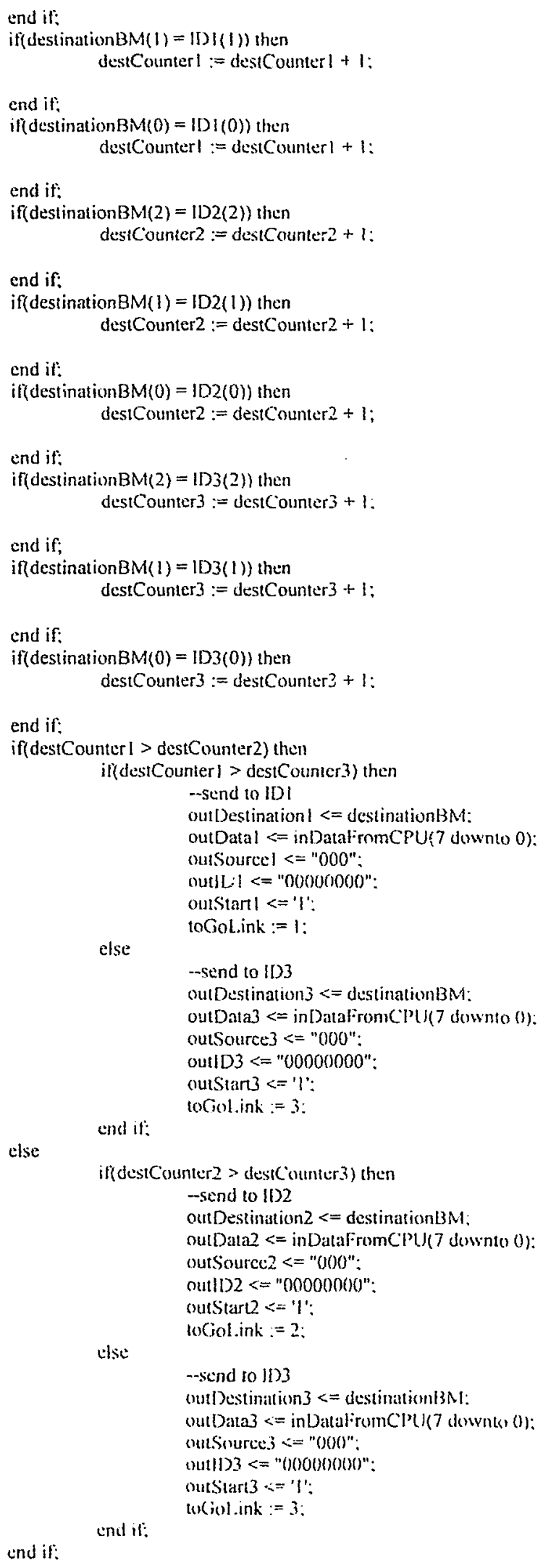

end if:

end il: 


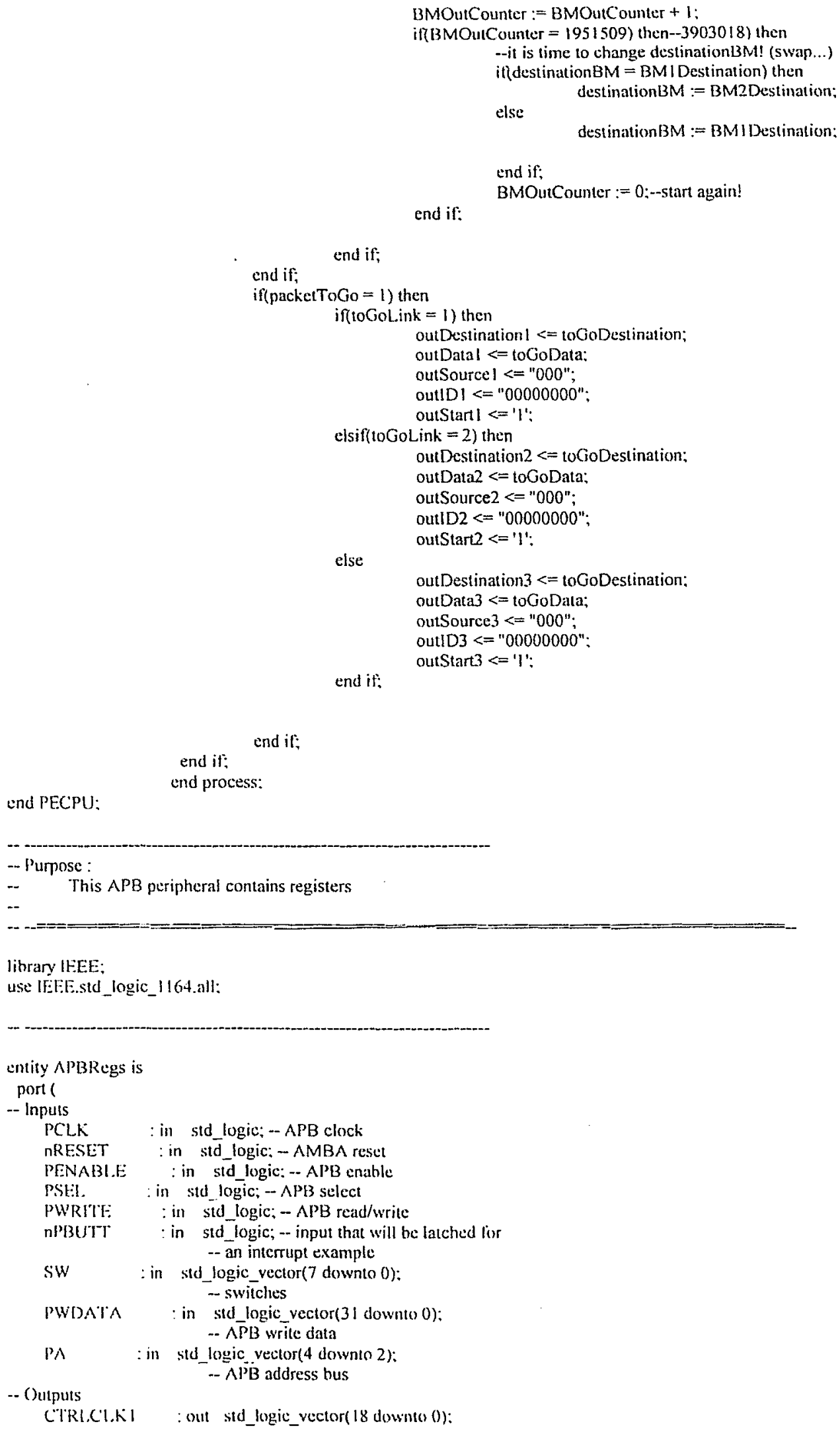

else

outDestination $3<=$ toGoDestination:

outData3 <= toGoData:

outSource $3<=$ "000";

outID3 $<=$ "00000000": outStart $3<=$ '1":

end if:

end if;

end if:

end PE.CPU:

end process:

-. Purpose :

This APB peripheral contains registers

library IFEE:

use IEEEE.std_logic_ 1 164.all: 


$$
\begin{aligned}
& \begin{array}{c}
\text {-- sets frequency of CLK1 } \\
\text { CIRI.CI.K2 : out std_logic_vector( } 18 \text { downto } 0) \text {; }
\end{array} \\
& \text { - sets frequency of CLK2 } \\
& \text { REGSINT : out std_logic:-intcrrupt output } \\
& \text { I.I:D : out std_logic_vector(8 downto 0); } \\
& \text { - L.ED control } \\
& \text { PRDATA : out std_logic_vector(31 downto } 0 \text { ) } \\
& \text {.- } \overline{A P B} \text { read data }
\end{aligned}
$$

\begin{tabular}{|c|c|c|}
\hline \multicolumn{3}{|l|}{ - Component declarations } \\
\hline $\begin{array}{c}\text { component PE_BlockMatch IS } \\
\text { PORT( }\end{array}$ & $\begin{array}{l}\text { clk } \\
\text { inDestination! } \\
\text { inSource ! } \\
\text { inDatal } \\
\text { inlD1 } \\
\text { inStart! } \\
\text { inDestination2 } \\
\text { inSource? } \\
\text { inData2 } \\
\text { inID2 } \\
\text { inStart2 } \\
\text { inDestination3 } \\
\text { inSource3 } \\
\text { inData3 } \\
\text { intD3 } \\
\text { inStart3 } \\
\text { mylD } \\
\text { ID1 } \\
\text { ID2 } \\
\text { ID3 } \\
\text { outDestimation! }\end{array}$ & 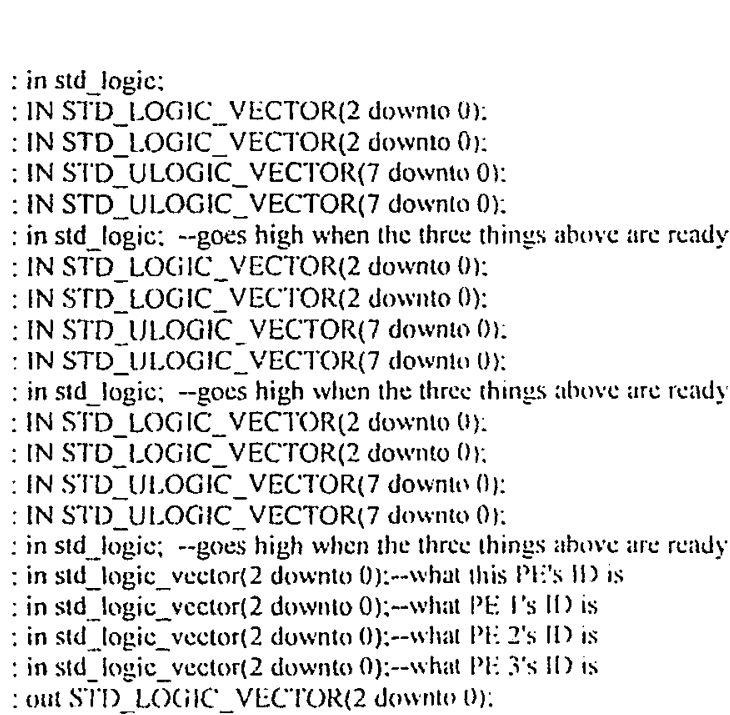 \\
\hline
\end{tabular}

end APBRRegs:

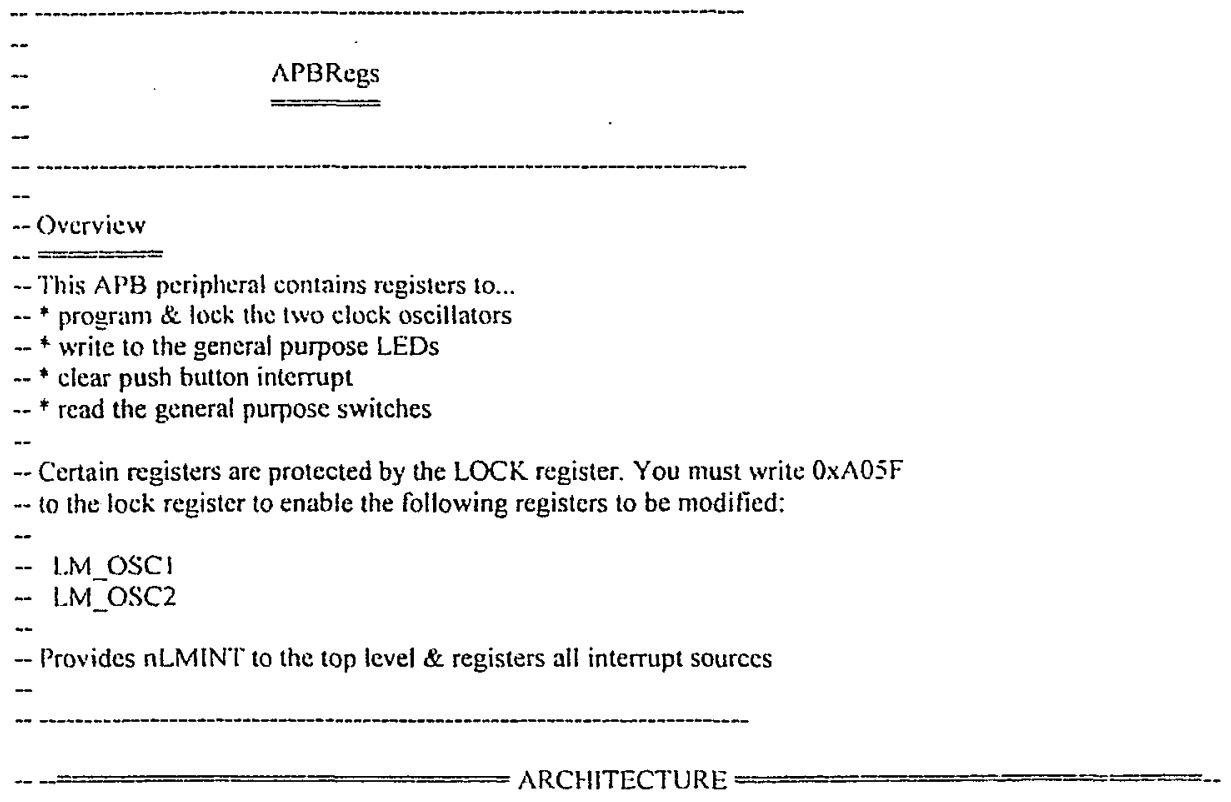

architecture synth of APBRegs is 
END component:

component PE_BM_Asscrt IS

PORTL

clk

inDestination

insourcel

inDatal

inID]

inStart

inDestination2

inSource2

inData2

inID2

insturt?

inDestination 3

inSource 3

inData3

inID3

inStart3

mylD

IDI

ID2

$1 \mathrm{D} 3$

outDestination

outSourcel

outDatal

out[D]

outStart I

outDestination2

outSource2

outData?

outlD2

outStart2

outDestination3

outSontrce3

outData3

outlD3

outStart:
TND component:

component PE_final_Assert IS

PORT' clk

inDestination

inSourcel

inDatal

inIDI

instart 1

inDestination2

insource?

indata2

inlD2

instan2

inDestination?
: out STD_LOGIC_VECTOR(2 downto 0):

: out STD_ULOGIC_VECTOR(7 downto 0):

: out STD_ULOGIC_VECTOR(7 downto 0):

: out std_logic:

out STD LOGIC VECTOR(2 downto 0$)$

: out STD_1.OGIC_VECTOR(2 downto 0$)$

: out STD_ULOGIC VECTOR(7 downto 0):

: ou STD_ULOGIC_VECTOR(7 downto 0):

: out std_logic;

: out STD_LOGIC_VECTOR(2 downto 0);

: out STD LOGIC_VECTOR(2 downto 0$)$;

: out STD_ULOGIC_VECTOR(7 downto 0$)$ :

: out STD_ULOGIC_VECTOR $(7$ downto 0$)$

: out std_Logic);

: in std_logic;

: IN STD LOGIC VECTOR(2 downio 0):

: IN STD_LOGIC_VIECTOR $(2$ downto 0$)$ :

: IN STD_ULOGIC_VECTOR(7 downto 0$)$;

: IN STD_ULOGIC_VECTOR(7 downto 0$)$;

: in std_logic; -goes high when the three things above are ready

: IN STD LOGIC_VECTOR(2 downto 0$)$;

: IN STD_LOGIC_VECTOR(2 downto 0):

: IN STD ULOGIC VECTOR(7 downto 0$)$;

: IN STD_ULOGIC_VECTOR(7 downto 0);

in std logic; -goes high when the three things above are ready

: IN STD_LOGIC_VECTOR(2 downto 0):

: IN STD_LOGIC_VECTOR(2 downto 0$)$;

: IN STD_ULOGIC_VECTOR(7 downto 0):

: IN STD_ULOGIC_VECTOR(7 downto 0);

: in std_logic; - -goes high when the three things above are ready

: in std_logic_vector(2 downto 0$)$; - what this PE's ID is

: in std_logic_vector(2 downto 0$)$; - what PE I's ID is

: in std_logic_vector(2 downto 0);--what PE 2's ID is

: in std_logic_vector( 2 downto 0$)$;-what PE 3 's ID is

: out STD LOGIC VECTOR(2 downto 0);

: out STD_LOGIC_VECTOR(2 downto 0):

: out STD_ULOGIC_VECTOR(7 downto 0);

: out STD_ULOGIC_VECTOR(7 downto 0);

: out std Jogic:

: out STD LOGIC VECTOR(2 downto 0):

: out STD_LOGIC_VECTOR(2 downto 0);

: out SID ULOGIC VECTOR(7 downto 0);

: out STD_ULOGIC_VECTOR(7 downto 0):

: out std logic;

: out STD_LOGIC_VECTOR(2 downto 0):

: out STD LOGIC VECTOR(2 downto 0$)$;

: out STD_ULOGIC_VECTOR(7 downto 0$)$

: out STD_ULOGIC_VECTOR(7 downto 0);

: out sid_logic); 


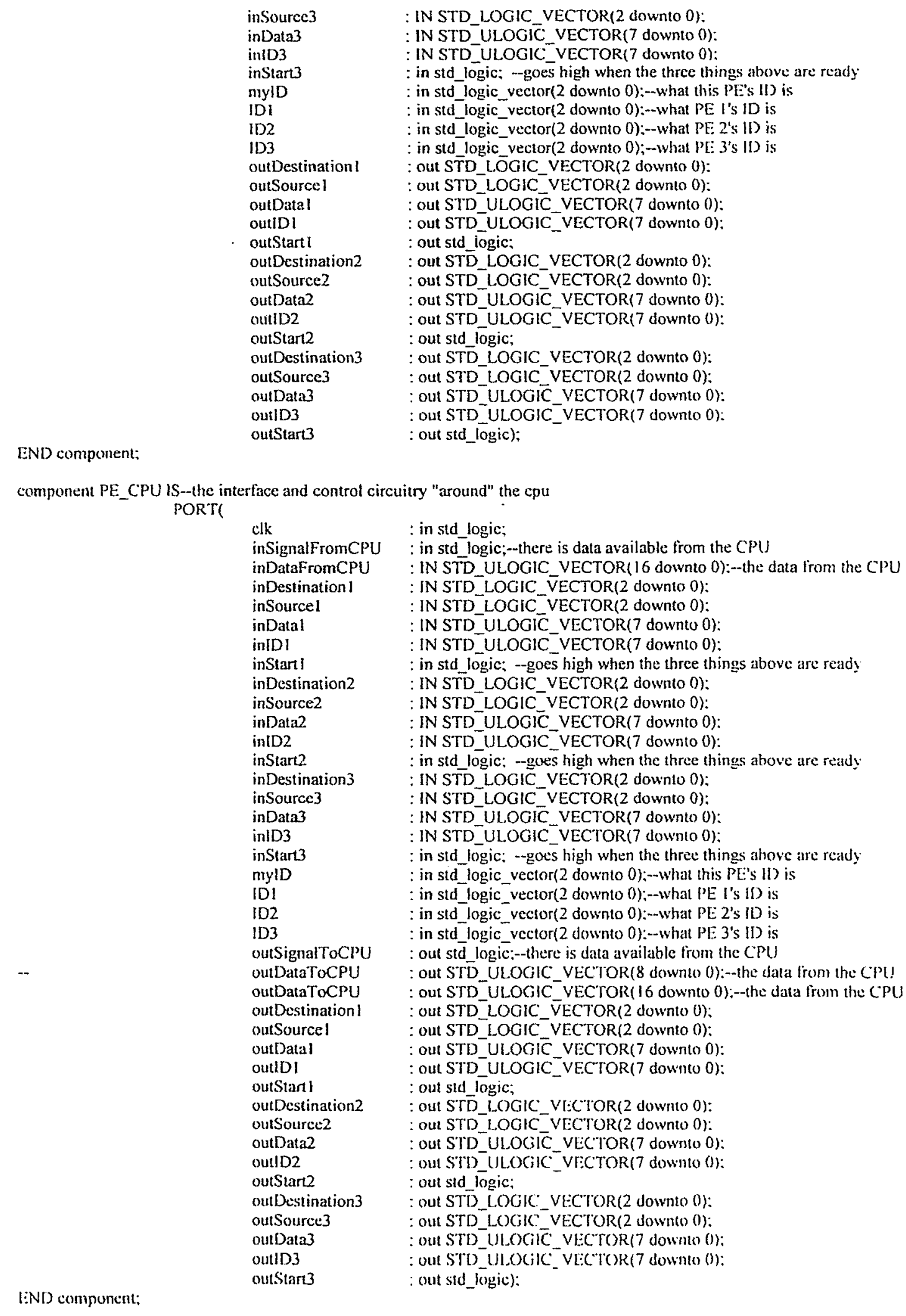

- Constant declarations 


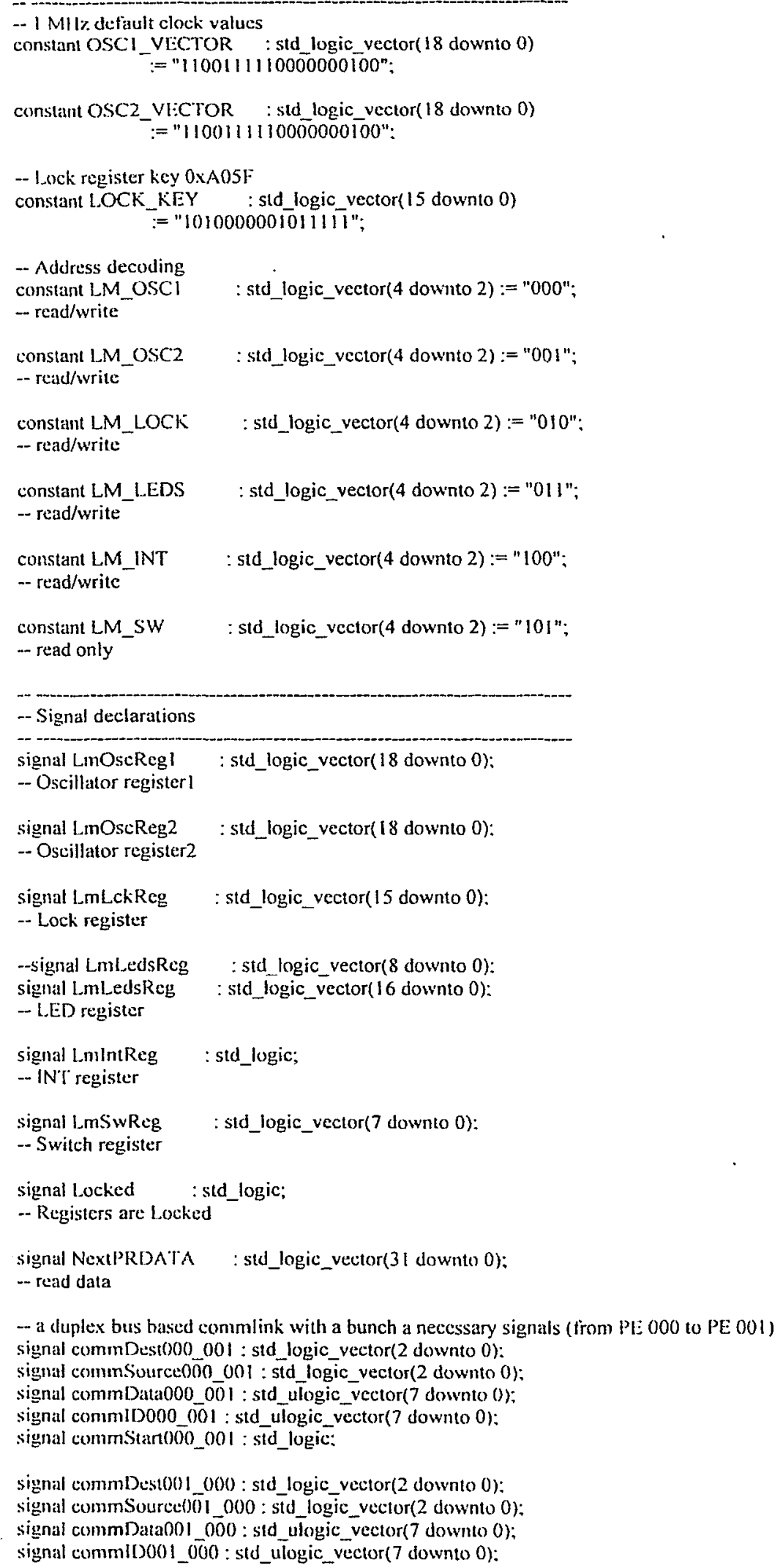


signal commStart001_000: std_logic:

-- a duplex bus based commlink with a bunch a necessary signals signal commDest000_010: std_logic_vector(2 downto 0): signal commSource 000 010: std logic vector(2 downto 0 ) signal commData000_010: std_ulogic_vector(7 downto 0$)$ : signal commiD000_010 : std_ulogic_vector 7 downto 0$)$; signal comm Stant000_010: sto_logic;

signal commDest010 $000:$ std_logic_vector $(2$ downto 0$)$; signal commSource010_000: std_logic_vector $(2$ downto 0$)$; signal commData0 10 000 : std ulogic vector( 7 downto 0 ): signal commiD0 10.000 : std_ulogic_vector(7 downto 0$)$; signal commStart0io_000: std_logic;

-- a duplex bus based commlink with a bunch a necessary signals signal commDest000 100 : std logic vector( 2 downto 0 ); signal commSource000_100: std_logic_vector(2 downto 0): signal commData000_100: std_ulogic_vector( 7 downto 0$)$; signal commID000_100: std_ulogic_vector(7 downto 0$)$; signal commStart000_100: std_logic;

signal conmbest $100 \_000$ : std_logic_vector(2 downto 0$)$; signal commSource100_000: std_logic_vector(2 downto 0 ); signal commDatal $00 \_000$ : std_ulogic_vector(7 downto 0); signal commid 100_000 : std_ulogic_vector(7 downto 0); signal commStart 100_000: std_logic;

-- a duplex bus based commlink with a bunch a necessary signals signal commDest001_101: std_logic_vector(2 downto 0 ); signal commSource 001 101: std logic vector $(2$ downto 0$)$; signal commData00I_101 : std_ulogic_vector $(7$ downto 0$)$; signal commlD001_101 : std_ulogic_vector(7 downto 0); signal commStart00!_101 : std_logic;

signal commDest 101_001: std_logic_vector(2 downto 0); signal commSource 101 [001 : std_logic_vector(2 downto 0): signal commDatal 01_001 : std_ulogic_vector(7 downto 0); signal commlD 101_001 : std_ulogic_vector(7 downto 0): signal commStart $101 \_001$ : std_logic:

-- a duplex bus based commlink with a bunch a necessary signals signal commDest00l 011 : std logic vector(2 downto 0 ); signal commSource00̄1_011: std_logic_vector(2 downto 0 ): signal commData001_011 : std_ulogic_vector(7 downto 0): signal commID001_011 : std_ulogic_vector(7 downto 0): signal commStart001_011: sid_logic;

signal commDest011_001: std_logic_vector(2 downto 0); signal commSource 011 001: std logic vector $(2$ downto 0 ): signal commData011_001: std_ulogic_vector(7 downto 0): signal commiD011_001 : std_ulogic_vector(7 downto 0): signal commStanol1_001 : std_logic;

- a duplex bus based commlink with a bunch a necessary signals signal comniDest010_011: std_logic_vector(2 downto 0): signal commSource010_011: std_logic_vector(2 downto 0 ): signal commData010 011 : std_ulogic_vector(7 downto 0): signal commlDo10_011 : std_ulogic_vector 7 downto 0 ): signal commStart010_011: std logic:

signal commbest011 010 : std_logic vector(2 downto (0); signal commSource 011 o10: sid_logic_yector(2 downto 0$)$ : signal commData0 11 010 : std ulogic vector(7 downto 0): signal commido II_010 : std_ulogic_vector(7 downto 0): signal cumnstartöl_010: sid_logic:

- il duplex bus based commlink with a bunch a necessary signals signal commDest010_110: std_logic_vector(2 downto 0): signall commSource 0 To_110: std_logic_vector(2 downto (1): 
signal commData0l0_110: std_ulogic_vector(7 downto 0); signal commlD0 $10 \quad \overline{1} 0$ : std_ulogic_vector(7 downto 0); signal commStartolo_110: std_logic;

signal commDest110_010: std_logic_vector(2 downto 0$)$; signal commSource $1 \overline{10} 010: \overline{s t d}$ logic vector $(2$ downto 0 ); signal commDatal10_010:std_ulogic_vector(7 downto 0): signal commlD 10 010:std_ulogic_vector(7 downto 0 ): signal commStart 1 10_010: std_logic:

-- a duplex bus based commlink with a bunch a necessary signals signal commDest $110 \_111$ : std_logic_vector(2 downto 0$)$; signal commSourcelio $111:$ std logic vector( 2 downto 0 ); signal commDatal10_\$1 : std_ulogic_vector(7 downto 0); signal commID 10 T/l : std ulogic_vector( 7 downto 0$)$ signal commStart $10 \_111:$ std_logic:

signal commDest111 110 : std logic vector(2 downto 0); signal commSource $] \overline{1}$ I_I $10: \overrightarrow{\text { std }}$ logic_vector(2 downto 0 ); signal commDatalll_ $\overline{110}$ : std_ulogic_vector( 7 downto 0$)$; signal conmID $\| 1 \_\lceil 0: \text { std_ulogic_vector(7 downto } 0)$; signal commStart $\overline{11} 110:$ std_logic;

-- a duplex bus based commlink with a bunch a necessary signals signal commDest 110 100: std_logic_vector(2 downto 0$)$; signal commSource $110 \_100:$ std_logic_vector( 2 downto 0$)$; signal commDatal $10 \_100:$ std_ulogic_vector(7 downto 0 ); signal commIDI 10_100: std_ulogic_vector(7 downto 0 ); signal commStart $1 \overrightarrow{10} 100: \overline{\text { std logic; }}$

signal commDestl00_110: std_logic_vector(2 downto 0); signal commSource $100 \quad 110:$ std logic_vector $(2$ downto 0$)$; signal commData 100_j $10:$ std_ulogic_vector(7 downto 0$)$; signal commID100_̄10 : std_utogic_yector(7 downto 0$)$ : signal commStart100_110: std_logic;

- a duplex bus based commlink with a bunch a necessary signals signal commDestl01_ $\|$ : std_logic_vector(2 downto 0$)$; signal commSourcelol_ $111:$ std_logic_veclor $(2$ downto 0$)$; signal commDatal01 III : std_ulogic_vector(7 downto 0); signal commiDi01_ $\overrightarrow{11}$ : std_ulogic_vector(7 downto 0); signal commStartion_ 111 : std logic;

signal commDest $111101:$ std logic vector(2 downto 0$)$; signal commSource $\bar{l} \bar{l}$ lol : std logic_vector(2 downto 0$)$; signal commDatall!_ $\overrightarrow{10} 1:$ sid_ulogic_vector $(7$ downto 0$)$; signal commlD 111 I01 : std_ulogic_vector(7 downto 0): signal commStart II $101:$ std_logic;

-- a duplex bus based commlink with a bunch a necessary signals signal commDesi100 $101:$ std_logic_vector(2 downto (b): signal commSource $100 \_101:$ std logic_vector(2 downto 0 ): signal commDatal00_101: std_ulogic_vector(7 downto 0 ); signal commlD $100 \quad \overline{10} 1$ : std ulogic_vector(7 downto 0$)$; signal commStart $100 \_101:$ std_logic:

signal conmDest101_100: std_logic_vector(2 downto 0); signal commSourcelol $100:$ std_logic_vector $(2$ downto 0$)$ : signal commDatal01 $100:$ std_ulogic_vector(7 downto 0$)$ : signal commIDI01 100 : std ulogic vector(7 downto 0); signal comm Start 100 __ 100 : std_logic:

-- a duplex bus based commlink with a bunch a necessary signals signal commDest0 11 III : std_logic_vector(2 downto 0): signal commSource $0 \overline{1} 1.11 ;$ std_logic_vector(2 downto 0$)$ : signal commidata0)! \II: std ulogic vector(7 downto 0): signal commlDo11_ 111 : std_ulogic_vector(7 downio 0): signal commStart011_111: std_logic;

signal coumDestll1_oil : std_logic_vector(2 downto 0); 
signal conmsource 11 _ 011 : std_logic_vector(2 downto 0$)$ : signal commDatal 11 0 11 : std ulogic vecior(7 downto 0); signal commlDlll_0ill: std_ulogic_vector( 7 downto 0): signal commStart 11 _ 011 : std_logic;

--signal dataToLEDs : std_ulogic_vector(8 downto 0$)$ : signal dataTol.EDs : std ulogic vector $(16$ downto 0$)$ : signal dataFrom LEDS : std_ulogic_vector $(16$ downto 0 ); signal signalTol_EDs : std_logic:

signal signalfromLEDs : std logic;

signal VAL000: std logic vector $(2$ downto 0$):=" 000 "$; signal VALO01 : std_logic_vector $(2$ downto 0$):=$ "001"; signal VALO10: std_logic_vector(2 downto 0$):=" 010^{\text {n: }}$ signal VALO11: std logic_vector(2 downto 0$):=011^{n}$. signal VALL100: std_logic_vector(2 downto 0$):=" 100 "$ ": signal VAL101: sld logic vector $(2$ downto 0$):=101 "$ ", signal VAL_110: std_logic_vector $(2$ downto 0$):=" 110 "$ signal VALIII: std_logic_vector $(2$ downto 0$):=" 111 "$ ":

- Function declarations

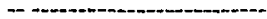

$-$

- Main body of code

-

begin

-if you are circuit 000 then your inputs start with 000 and your outputs end with 000

PE000: PE_CPU port map(PCLK, signalFromLEDs, dataFromLEDs, commDest000_001. commSource000_001.

commDala000_001, commID000_001, commStar000_001.

commDest000_100, commSouree000_100, commData000_100,comm10000_100,commStart 000 100, commDest000_010, commSource 000_010, commData000_010, commiD000_010. commStart000_010. VALO00, VALO0 I, VAL100, VALO10, signalToLEDs, dataTOLEDs. commDest001_000, commSource001_000, commData001_000, commID001_000. commStart001_000. commDest 100_000, commSource 100_000, commData 100_000, commiD 100_000, commStarl100_000. commDest010_000, commSource010_000, commData010_00, commlD010_000, commStart010_000):

PE00I: PE_BlockMatch port map(PCLK, commDest001_101, commSource001_101, commData001_101, commID001_101. commStart001 101. commDest001_011, commSource001_011.commData001_011.commID001_011.commStart001_011. commDest001_000, commSource001_000, commData001_000, commID00I_000.commStart001_000. VALO01, VALIOI, VALOII, VALOOO, commDest101_C01, commSource101_001,commData101_001.commID101_001.commStart101_001. commDest011_001, commSource011_001, commData011_001, commID011_001. commStano11_001. commDest000_001, commSource000_001, commData000_001, commID000_001, commStartoo0_001):

PE() 10: PE_BlockMatch port map(PCLK, commDes1010 000, commSource010 000, commData010 000, commID010 000. commStart) 10000 commDest010_011, commSource010_011, commData010_011, commID010_011. commStart(010_011. commDest010_110, commSource 010_110, commData010_110, commiD010_110, commStart010_110. VALOIO, VALOOO, VALOII, VALIIO. commDest000_010, commSource000_010, commData000_010.commIS000_010. commStart000_010.

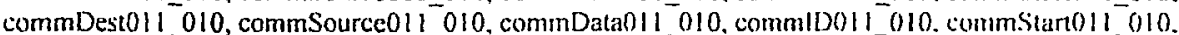
conumDest110_010, commSourcel10_010.commDatal10_010.commlD110_010. commstartllo_(1)10):

PEO11: PE:BM_Assert port map(PCLK,

commDest011_010,commSource011_010,commData011_010.commlD011_010. commStart011_010. commDest011_111,commSource011_111.commDatioli_III.commiDoli_III.commStartol1_ 11 . commDest011_001,commSource011_001.commData011_001.commID011_001.commSiarr(011_001. VALOII, VALOIO, VAIIII, VALOOI. commDest010_011, commSource010_011, commData010_011, conml1)010_011, commStart(1)10_(11).

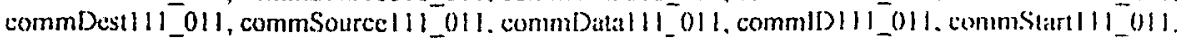

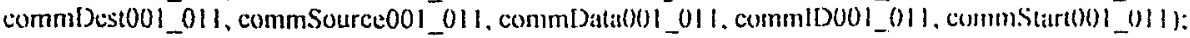

P1:100: Pl:_Final_Assert port map(PCLK.

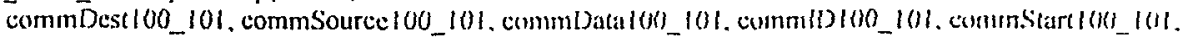


commDest 100_000,commSourcel00_000,commData100_000,commID100_000,commStart100_000. commDest $100-110$, commSource $100^{-} 110$, commData 100_110, comm10100_110, commStart100_110. VALI00, VALTOI, VALOOO, VALITO

commDest101 100, commSource 101_100, commDatal01_100, commlD101_100, commStart101 100, commDes1000_100, commSource000_100, commData000_100, commlD000_100, commStar1000_100, commDest 110_100,commSource 110_100,commDatal10_100, commiD 110_100, commStart 110_100):

PE101: PE: BlockMatch port map(PCLK,

commDestl01 001, commSource 101 001, commDatal01 001, commID101_001, commstart101_001. commDest101_111, commSource 101_11, commDatal01_111, commID101_111, commStar101_111, commDestI01_100, commSourcel01_100, commDatal01_100,commIDI01_100, commStart 101 _ 100 , VALI01, VALO01, VAL_III, VAL100,

commDest001_101, commSource001_101, commData001_101, commID001_101, commStart001_101, commDest111_101, commSource 11 _101, commDatal11_101, commiDI11_101, commStart11!_101, commDest100_101, commSource100_101, commData100_101, commID100_101, commStart100_101);

PE1 10: PE_BlockMatch port map(PCLK,

commDest 110_010.commSource 110_010, commDatal10_010,commlD110_010, commStart1 10_010. commDest 110 111 , commSource 110-111, commDatal10-111, commiD $110^{-} 111$, commStart $110^{-} 111$. commDest 1 10_100, commSource 10_100, commDatal 10_100, commIDI 10_100, commStart110_100. VALI10, VALOI0, VALIII, VALI00,

commDest010_110.commSource010_110. commData010_110, commID010_110, commStart010_110. commDest 11_ 110, commSource 111_110. commDatal11_110, commlDI11_110. commStart111_110. commDest 100_110, commSource 100_110, commData100_110, commID100_110, commStart100_110);

PEIII: PE BlockMatch port map(PCLK.

commDest111_101,commSource 111_101, commDatal11_101,commIDI11_101,commStart111_101. commDest111011, commSource 111_011, commDatal11_011, commiD111_011, commStart 111,011, commDest111_110, commSource 111_110, commData 11]_110. commID 11]_110, commStart111_ 110 . VALI11, VALI01. VALOII, VALI10,

commDest101_111, commSource101_111, commDatal01_111,comm[D101_111,commStar101_111, commDest011_11, commSource011_111, commData011_111, commlD011_111, commStart011_ 11 , commDest 10_111, commSource 110_111, commDatal10_111, commID 110_111, commStart 110_11);

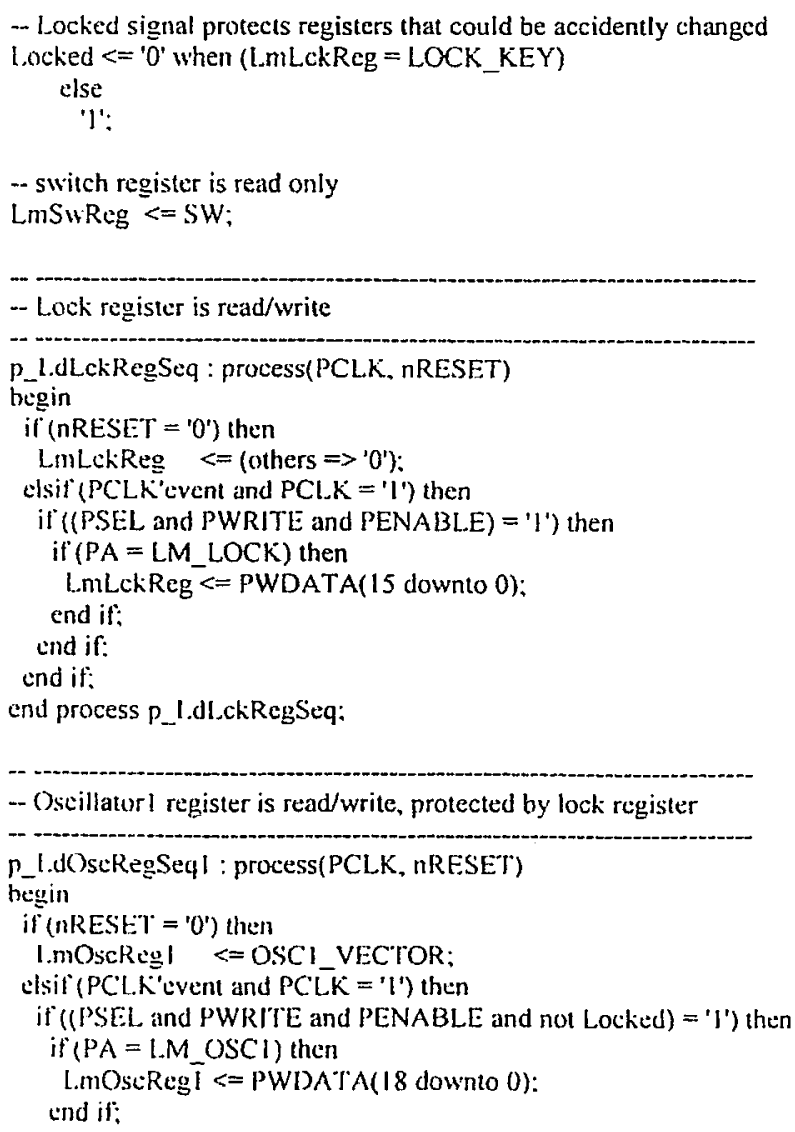




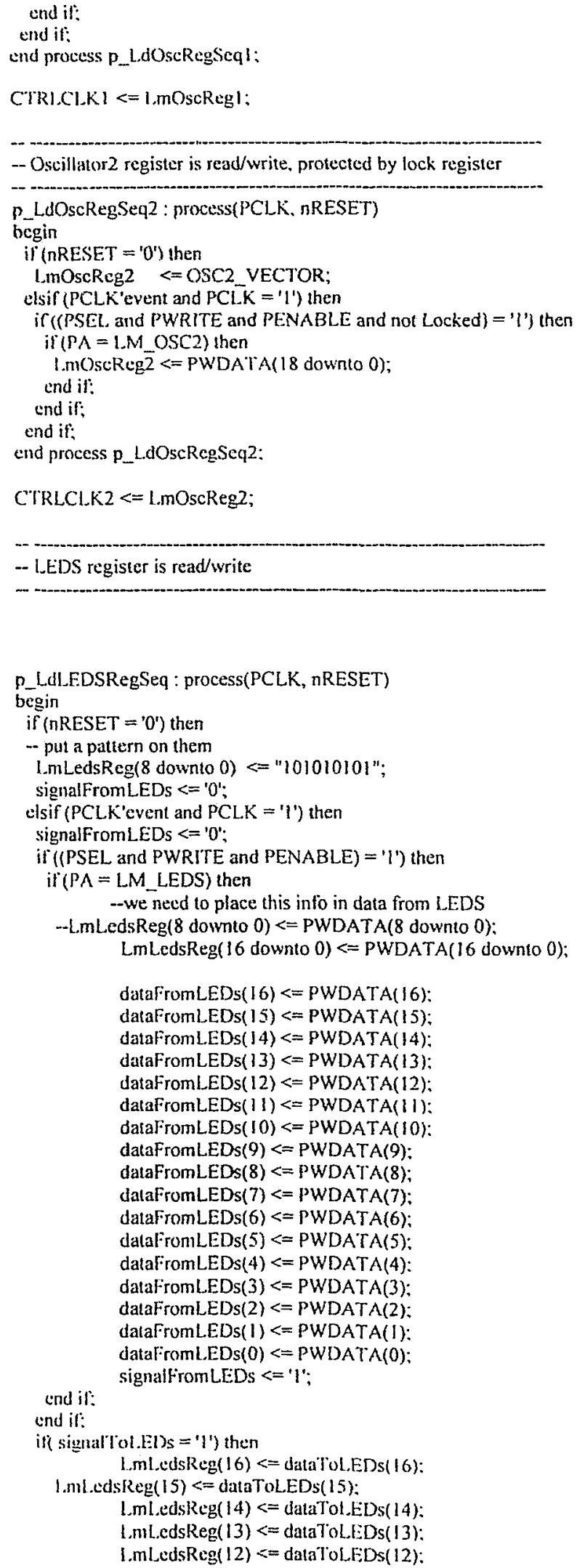




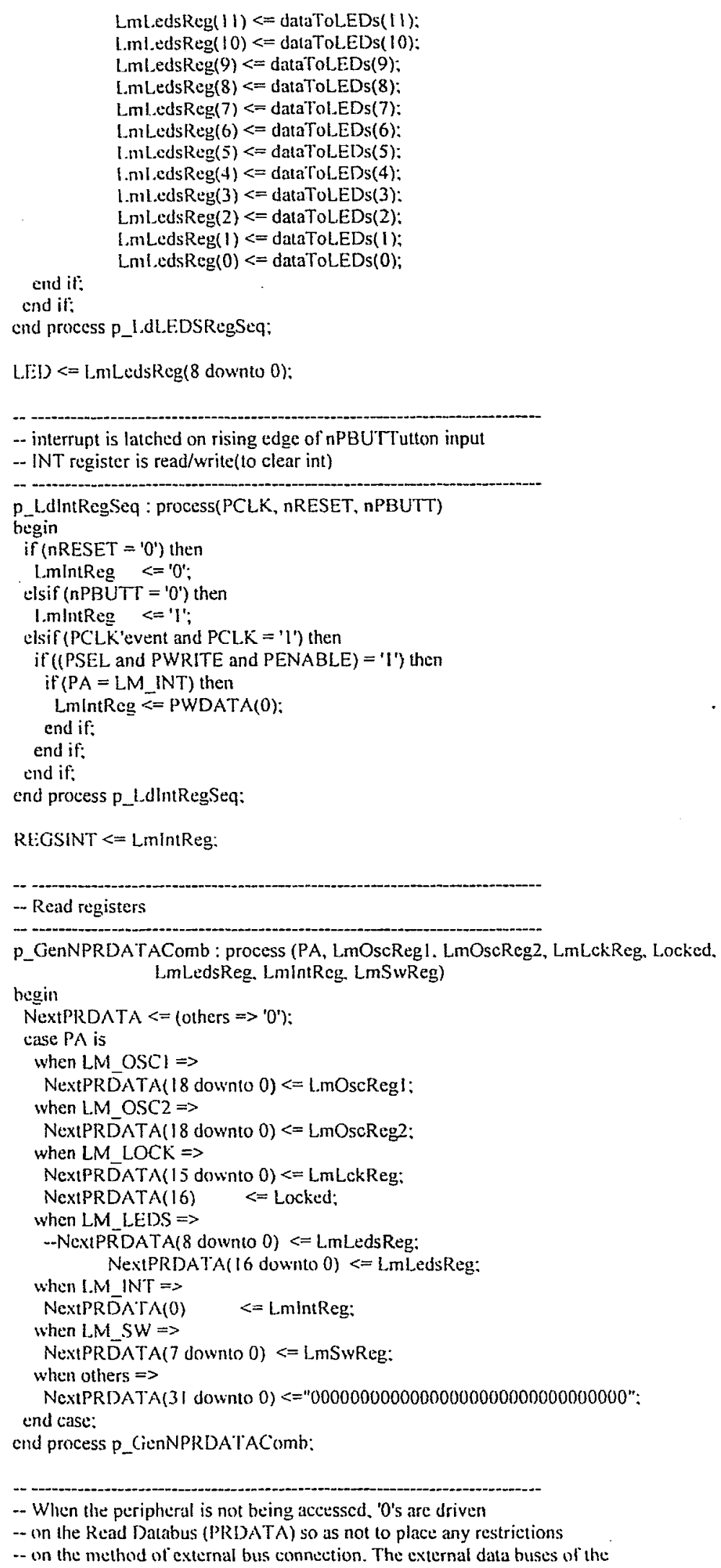




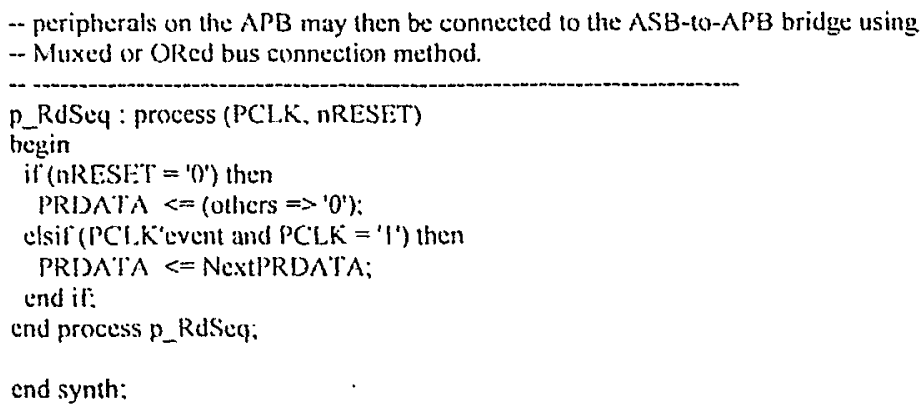

\#deline LM_LFDS 0xC000000C//0xC0000008//temp set to lock register instead// $0 \times C 000000$--end with $8=10 \mathrm{ck}$ reg. end with $\mathrm{C}=\mathrm{LE} \overline{\mathrm{D}}$

/* Timer register intormations*/

$\begin{array}{lll}\text { \#define } & \text { TIMERI_CTRL } & (0 \times 13000108) \\ \text { Hdeline } & \text { TIMERI_VALUE } & (0 \times 13000104) \\ \text { \#define } & \text { TIMERI_LOAD } & (0 \times 13000100) \\ \text { \#deline } & \text { TIMER)_CLR } & (0 \times 1300010 \mathrm{C}) \\ \text { Hdetine } & \text { TIMIER2_LOAD } & (0 \times 13000200)\end{array}$

/* Register set for IRQ controller ... pp 4-32*/

\#define IRQ S.TATUS

$(0 \times 14000000)$

\#define IRQ_RAWSTAT (0x14000004)

\#define IRQ ENABLESET $\quad(0 \times 14000008)$

\#detine IRQ_ENABLECLR (0x1400000C)

/* hit assignment for interrupts for interrupt controlles 0 (first CPU) */

\#detine SOFIINT $(0 \times 1<0)$

\#detine TIMERINTI $\quad(0 \times 1<<6)$

\#detine TIMERINT2 $\quad(0 \mathrm{x}) \ll 7)$

1 * IRQ Vector address for Integrator/AP platform */

\#detine IRQ_VH:Cl (0.18)

Hdetine AND HIL:TER Ox0000FFFF//0x0000003F

unsigned char activeframe[FRAME_DIMENSIONI[FRAME_DIMLENSION]:

unsigned chir

oldFrameArea[FRAME_DIMENSION+2*SCANNING_RADIUSIIFRAMH_DIMENSION+2*SCANNINCI_RADILISI: unsigned char wholelnage IJIMAGE_ROWS][IMAGE COLS]:

unsignted char wholcimagc2[IMAGE_ROWS][IMAGE_COLSW:

unsigned char bitmapleader Infol $1+1$ :

unsigned char intiolteaderinto 40$\}$ : 


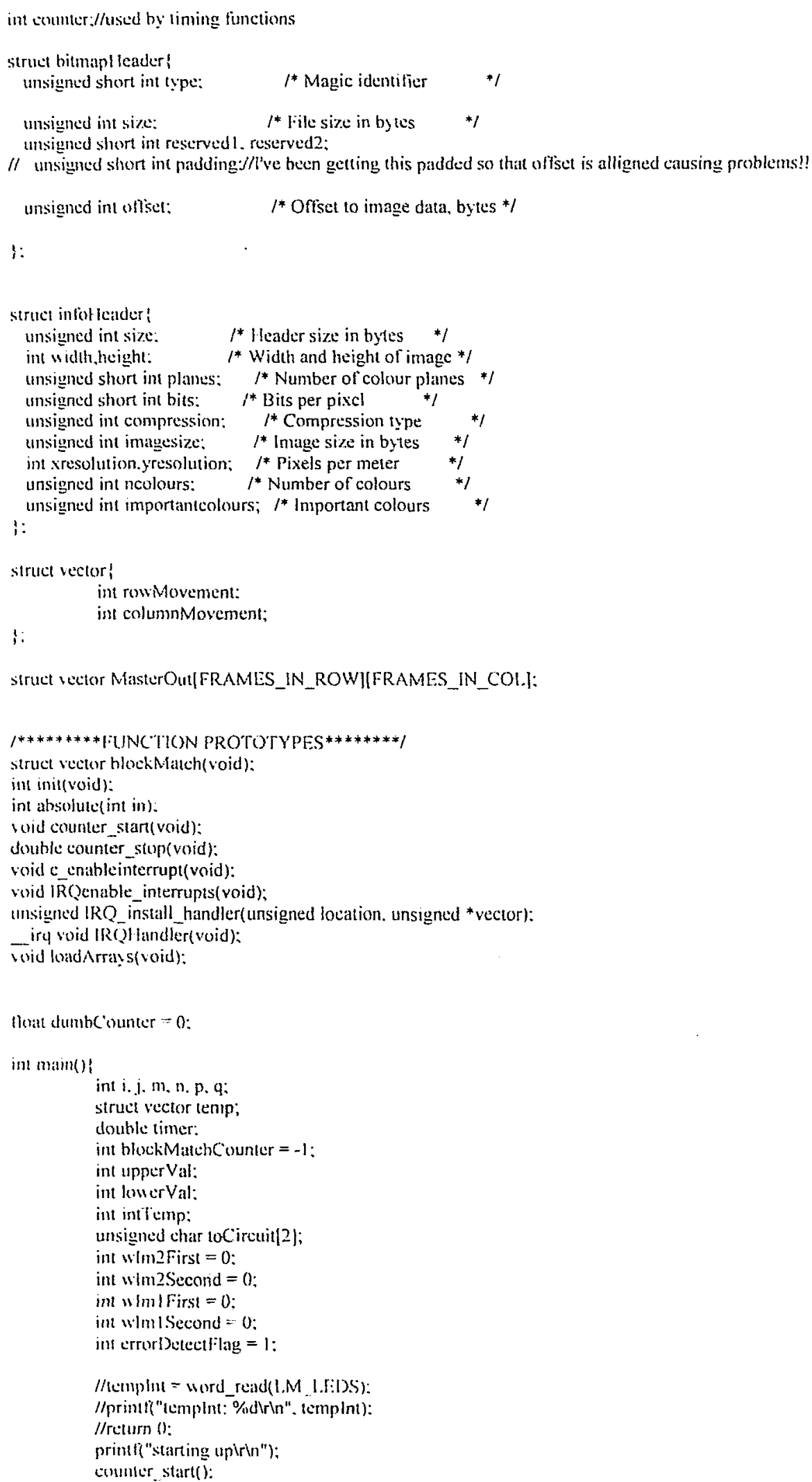




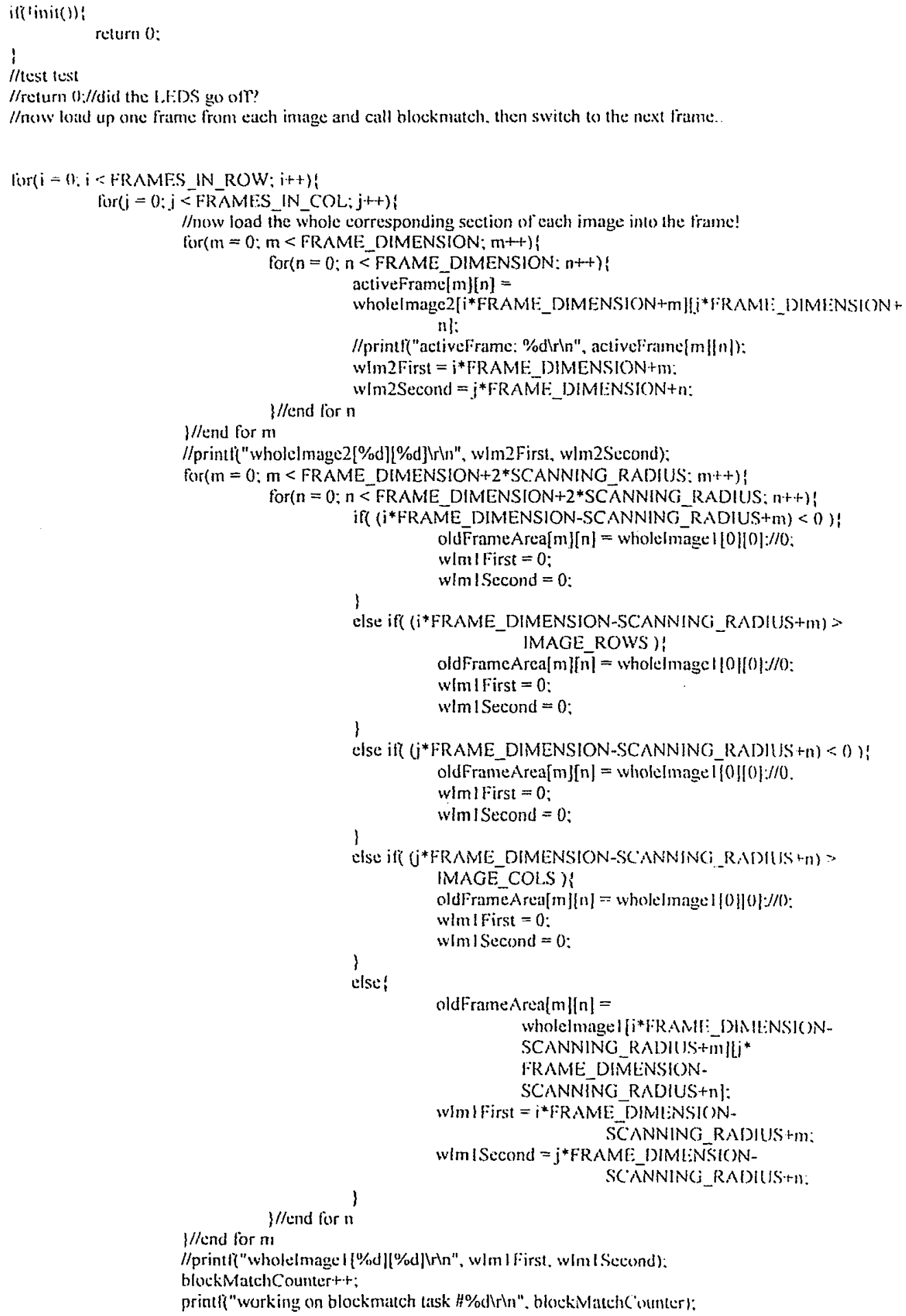

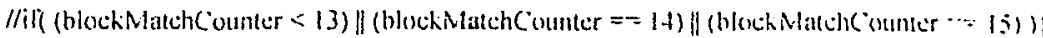

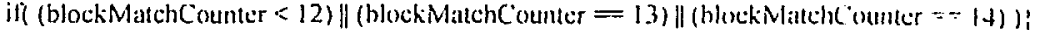
//thell do it in IIW 


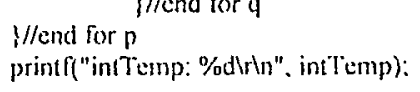

fortq $=0 ; q<\left(2 *\right.$ SCANNING_RADIUS+1): $\left.\left.q^{+}+r\right)\right\}$ For $(m=0 ; m<$ FRAMIE_DIMIENSION: $m++) 4$

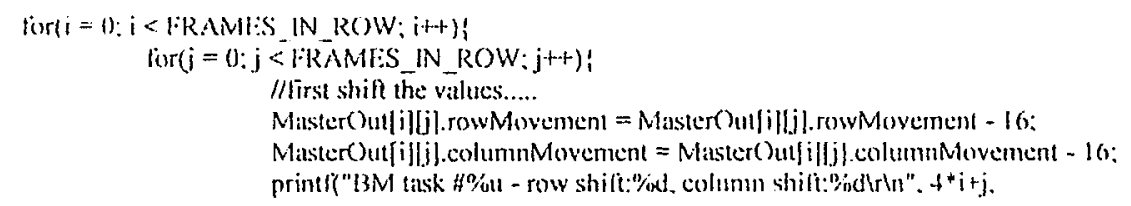




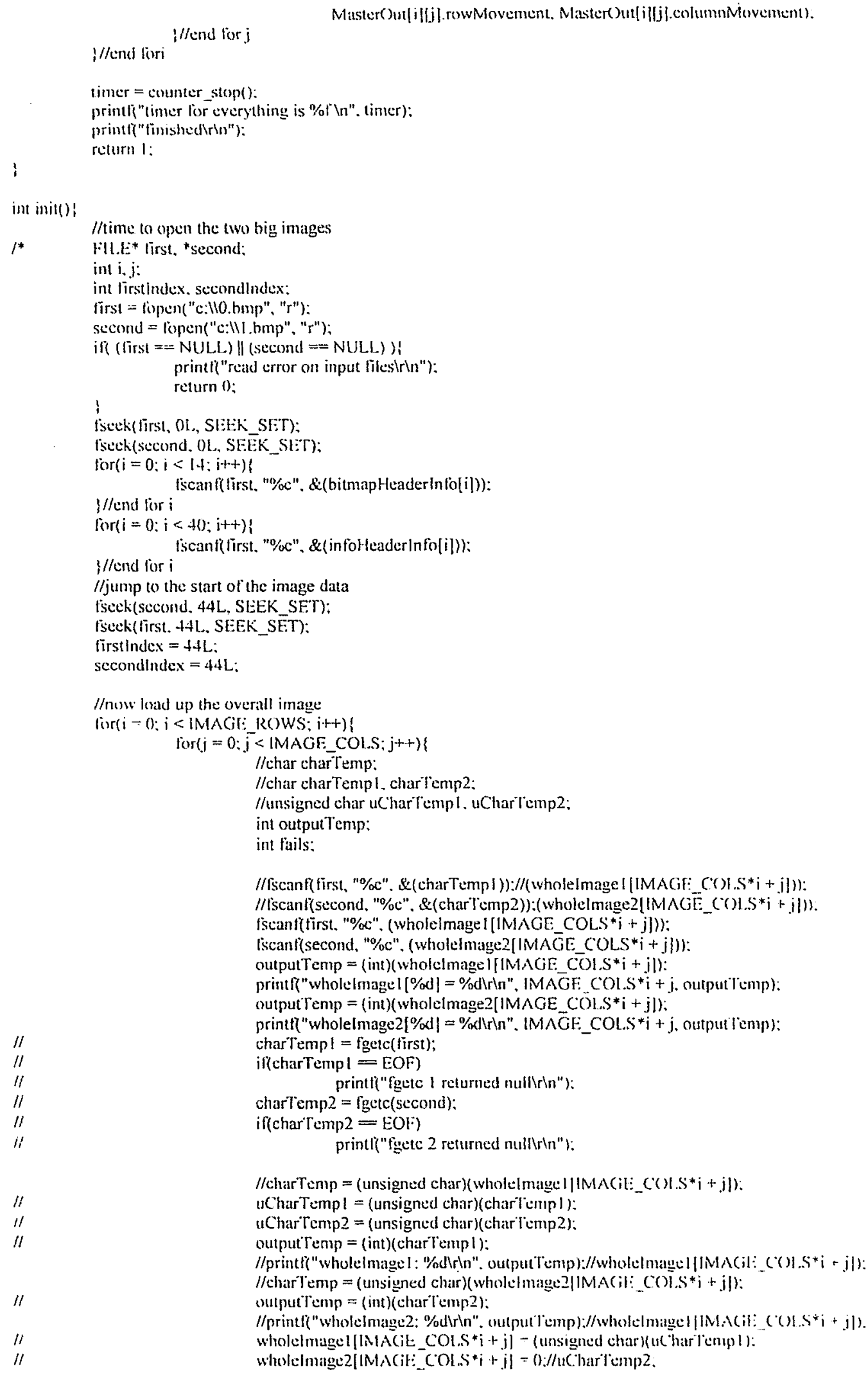




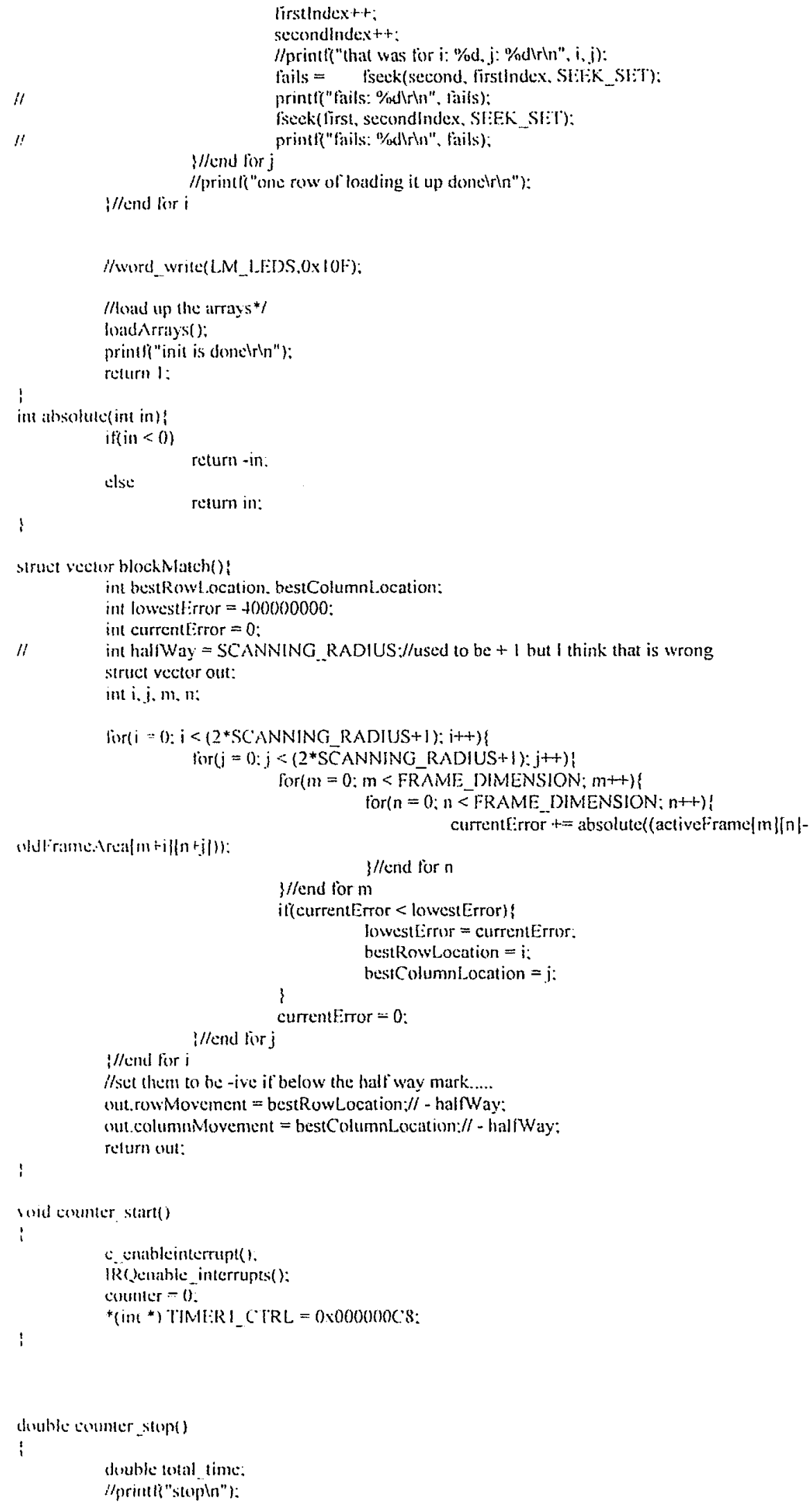




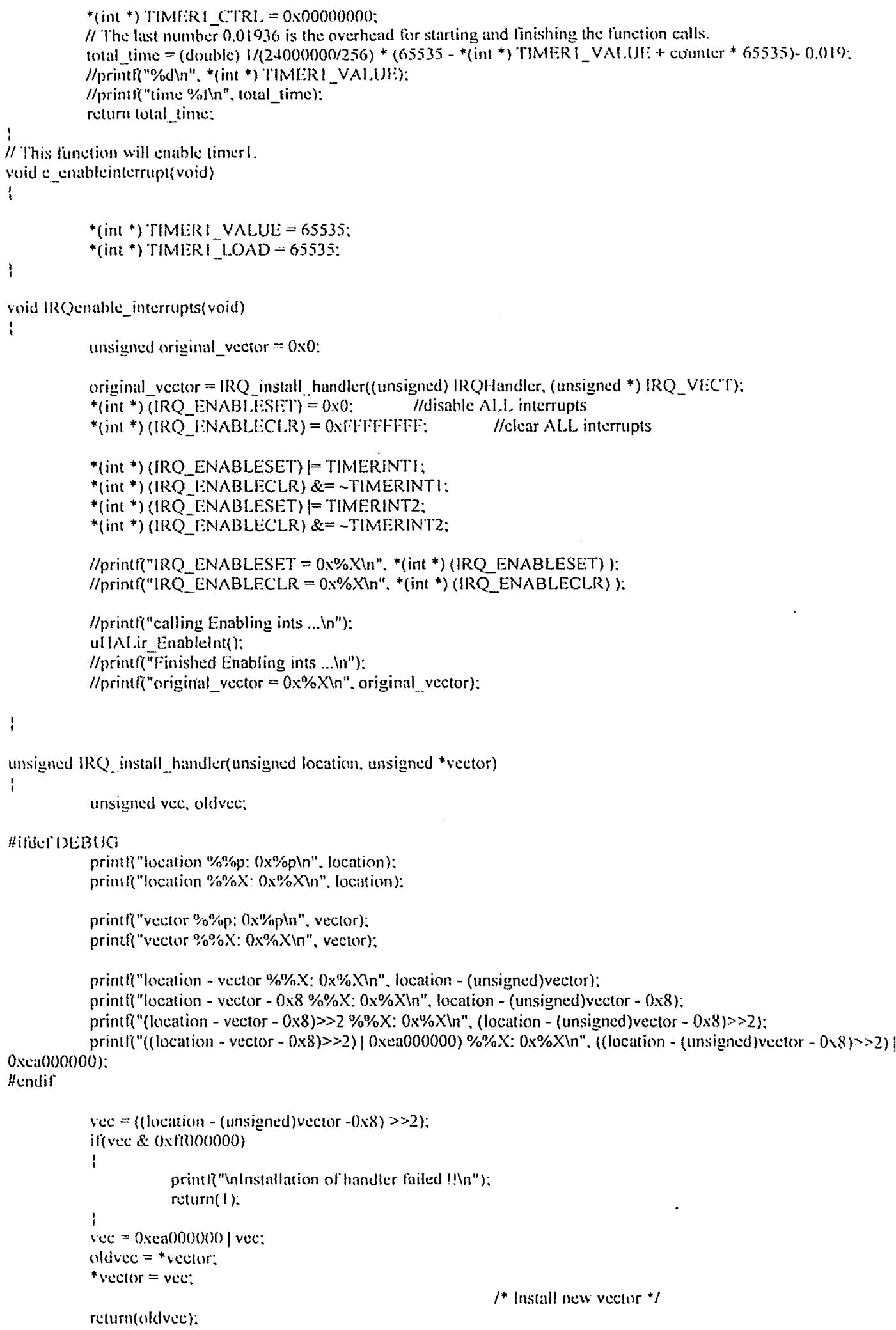


ire void IRQP landler(void)

I/int n:

unsigned int *base $=$ (unsigned int *) IRQ STATUS; I*IRQ status register for processer $0 *$ *I

$I^{*}$ Determine interrupt type and call appropriate handler */

if * base \& TIMI:RINT!)

* (int *) TIMERI_CTRL $=0 \times 00000000$

countert+r:

//for $(n=0 ; n<10 ; n++)$

//printt("\%din", *(int *JTIMERI_VALUL:):

*(int *) TIMIERI CLR $=0$;

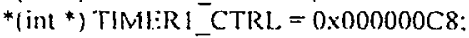

;

Reproduced with permission of the copyright owner. Further reproduction prohibited without permission. 